\title{
Gamma-Ray Bursts in the Swift Era
}

\author{
N. Gehrels ${ }^{1}$ E. Ramirez-Ruiz, ${ }^{2}$ And D. B. Fox ${ }^{3}$ \\ [1] NASA-Goddard Space Flight Center, Greenbelt, Maryland 20771; email: \\ gehrels@milkyway.gsfc.nasa.gov
}

[2] Department of Astronomy and Astrophysics, University of California, Santa Cruz, California 95064; email: enrico@ucolick.org

[3] Department of Astronomy and Astrophysics, Pennsylvania State University, University Park, Pennsylvania 16802; email: dfox@astro.psu.edu

\begin{abstract}
Key Words cosmology: early universe; galaxies: interstellar medium, highredshift; gamma rays: observations, theory; stars: Wolf-Rayet; neutrinos; supernovae: general; gravitational waves
\end{abstract}

\begin{abstract}
With its rapid-response capability and multiwavelength complement of instruments, the Swift satellite has transformed our physical understanding of $\gamma$-ray bursts (GRBs). Providing highquality observations of hundreds of bursts, and facilitating a wide range of follow-up observations within seconds of each event, Swift has revealed an unforeseen richness in observed burst properties, shed light on the nature of short-duration bursts, and helped realize the promise of GRBs as probes of the processes and environments of star formation out to the earliest cosmic epochs. These advances have opened new perspectives on the nature and properties of burst central engines, interactions with the burst environment from microparsec to gigaparsec scales, and the possibilities for nonphotonic signatures. Our understanding of these extreme cosmic sources has thus advanced substantially; yet, more than 40 years after their discovery, GRBs continue to present major challenges on both observational and theoretical fronts.
\end{abstract}

\section{CONTENTS}

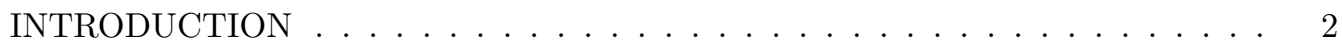

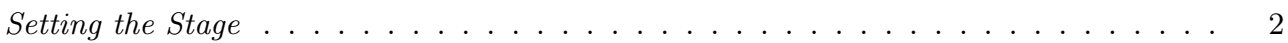

A Burst of Progress . . . . . . . . . . . . . . . . . . . 3

WHAT IS A GAMMA-RAY BURST? . . . . . . . . . . . . . . 6 6

BURST AND AFTERGLOW OBSERVATIONS . . . . . . . . . . . . . 10

Prompt High-Energy Emission . . . . . . . . . . . . . . . . . . . 10

Afterglow Observations . . . . . . . . . . . . . . . . . . . . . . . 13

Interpreting Prompt and Afterglow Emission. . . . . . . . . . . . . 22

The Radiated Energy Inventory . . . . . . . . . . . . . . . . . . . . . . . . 23 
ENVIRONMENTS AND HOST GALAXIES . . . . . . . . . . . . . . . 25

Cosmological Setting . . . . . . . . . . . . . . . . . . . . . . 25

Host Galaxies of Long Bursts . . . . . . . . . . . . . . . . . . . . . . 26

Host Galaxies of Short Bursts . . . . . . . . . . . . . . . . . . . 31

Neither Long Nor Short . . . . . . . . . . . . . . . . . . . . . . . . . 333

BASIC PHYSICAL CONSIDERATIONS . . . . . . . . . . . . . 33

The Central Engine . . . . . . . . . . . . . . . . . . . 34

Accretion Flows . . . . . . . . . . . . . . . . . . . . . 35

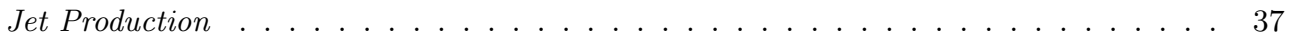

Jet Collimation, Stability, and Confinement . . . . . . . . . . . . 38

Dissipation and Cooling Effects Within the Jet . . . . . . . . . . . . 40 40

Jet Interaction with the External Environment . . . . . . . . . . . . . . . . . . . . 41

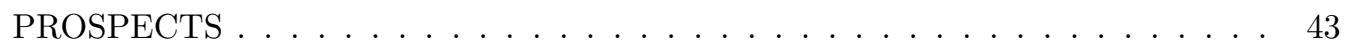

Facilities. . . . . . . . . . . . . . . . . . . . . . 43

Multimessenger Aspects . . . . . . . . . . . . . . . . 45

Cosmology . . . . . . . . . . . . . . . . . . . . 46

Theoretical Prospects . . . . . . . . . . . . . . . . . . 47

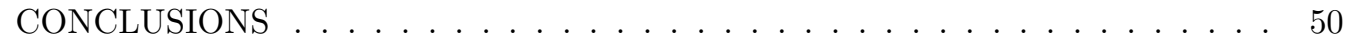

\section{INTRODUCTION}

\subsection{Setting the Stage}

Gamma-ray bursts (GRBs) are among the most fascinating phenomena in the Universe. They are bright flashes of radiation with spectral energy distributions peaking in the $\gamma$-ray band. They have durations measured in seconds and appear to be capable of producing directed flows of relativistic matter with kinetic luminosities exceeding $10^{53} \mathrm{erg} \mathrm{s}^{-1}$, making them the most luminous events known. All evidence points to a gravitational power source associated with the cataclysmic formation of a relativistic star or to a precursor stage whose inevitable end point is a stellar mass black hole.

The field of GRB astronomy has been greatly stimulated by the launch of the Swift satellite (Gehrels et al. 2004) in 2004 with its rapid response and panchromatic suite of instruments onboard, and by the development of new-technology robotic telescopes on the ground. A multidisciplinary approach is now emerging with data combined across the electromagnetic spectrum to learn about the physical processes at play; "spectral chauvinism" can no longer be tolerated in the modern study of GRBs. Even non-photonic neutrino and gravitational wave instruments are becoming more sensitive and may soon be detecting signatures related to GRBs.

While interesting on their own, GRBs are now rapidly becoming powerful tools to study detailed properties of the galaxies in which they are embedded and of the universe in general. Their apparent association with massive star formation and their brilliant luminosities make them unique probes of the high-redshift universe and galaxy evolution. Absorption spectroscopy of GRB afterglows is being used to study the ISM in evolving galaxies, complementary to the traditional studies 
of quasar absorption line systems. Possibly the most interesting use of GRBs in cosmology is as probes of the early phases of star and galaxy formation, and the resulting reionization of the universe at $z \sim 6-20$. GRBs are bright enough to be detectable, in principle, out to much larger distances than those of the most luminous quasars or galaxies detected at present. Thus, promptly localized GRBs could serve as beacons which, shining through the pregalactic gas, provide information about much earlier epochs in the history of the universe.

Before the advent of Swift, the study of GRBs had evolved somewhat unsystematically, and, as a result, the field has a great deal of historical curiosities such as complex classifications schemes which are now becoming streamlined as the field matures. Objects which were once thought to be different are now found to be related and the style of research has shifted from piecewise studies to a more general statistical approach. Although leaps in understanding can still come from extraordinary events as we show in several examples in this review, the applications to broader astrophysics are coming from the compilations of hundreds of events. The literature on this subject has therefore become quite large, and we apologize for referring now and then only to the most recent comprehensive article in a given topic. There are several recent summary articles that give excellent reviews in specific areas related to GRBs. These include the supernova-burst connection (Woosley and Bloom 2006), short GRBs (Lee and Ramirez-Ruiz 2007, Nakar 2007a), afterglows (van Paradijs et al. 2000, Zhang 2007) and theory (Mészáros 2002). Our objective here is to summarize the field of GRB astronomy, from the Swift era and prior to the next steps with the Fermi Gamma Ray Observatory (Atwood et al. 2009), interpreting past findings while looking ahead to future capabilities and potential breakthroughs.

\subsection{A Burst of Progress}

The first sighting of a GRB came on July 2, 1967, from the military Vela satellites monitoring for nuclear explosions in verification of the Nuclear Test Ban Treaty (Klebesadel et al. 1973). These $\gamma$-ray flashes, fortunately, proved to be different from the man-made explosions that the satellites were designed to detect and a new field of astrophysics was born. Over the next 30 years, hundreds of GRB detections were made. Frustratingly, they continued to vanish too soon to get an accurate angular position for follow-up observations. The reason for this is that $\gamma$-rays are notoriously hard to focus, so $\gamma$-ray images are generally not very sharp.

Before 1997, most of what we knew about GRBs was based on observations from the Burst and Transient Source Experiment (BATSE) on board the Compton Gamma-Ray Observatory, whose results have been summarized in Preece et al. (2000). BATSE, which measured about 3000 events, revealed that between two and three visible bursts occur somewhere in the universe on a typical day. While they are on, they can outshine every other source in the $\gamma$-ray sky, including the Sun. Although each is unique, the bursts fall into one of two rough categories. Bursts that last less than two seconds are short, and those that last 
longer - the majority - are long. The two categories differ spectroscopically, with short bursts having relatively more high-energy $\gamma$-rays than long bursts do.

Arguably the most important result from BATSE concerned the distribution of bursts. They occur isotropically - that is, evenly over the entire sky - suggesting a cosmological distribution with no dipole and quadrupole components. This finding cast doubt on the prevailing wisdom, which held that bursts came from sources within the Milky Way. Unfortunately, $\gamma$-rays alone did not provide enough information to settle the question for sure. The detection of radiation from bursts at other wavelengths would turn out to be essential. Visible light, for example, could reveal the galaxies in which the bursts took place, allowing their distances to be measured. Attempts were made to detect these burst counterparts, but they proved fruitless.

A watershed event occurred in 1997, when the BeppoSAX satellite succeeded in obtaining high-resolution X-ray images (Piro et al. 1999) of the predicted fading afterglow of GRB 970228 - so named because it occurred on February 28, 1997. This detection, followed by a number of others at an approximate rate of 10 per year, led to positions accurate to about an arc minute, which allowed the detection and follow-up of the afterglows at optical and longer wavelength: ${ }^{1}$ (e.g., van Paradijs et al. 1997). This paved the way for the measurement of redshift distances, the identification of candidate host galaxies, and the confirmation that they were at cosmological distances (Metzger et al. 1997).

Among the first GRBs pinpointed by BeppoSAX was GRB 970508 (Metzger et al. 1997). Radio observations of its afterglow provided an essential clue. The glow varied erratically by roughly a factor of two during the first three weeks, after which it stabilised and then began to diminish (Frail et al. 1997). The large variations probably had nothing to do with the burst source itself; rather they involved the propagation of the afterglow light through space. Just as the Earth's atmosphere causes visible starlight to twinkle, interstellar plasma causes radio waves to scintillate. Therefore, if GRB 970508 was scintillating at radio wavelengths and then stopped, its source must have grown from a mere point to a discernible disk. "Discernible" here means a few light-weeks across. To reach this size the source must have been expanding at a considerable rate - close to the speed of light (Waxman et al. 1998).

The observational basis for a connection between GRBs and supernovae was prompted by the discovery that the BeppoSAX error box of GRB 980425 contained supernova SN 1998bw (Galama et al. 1998). A number of other GRBs have since shown a 1998bw-like temporal component superposed on the power law optical lightcurve (Woosley and Bloom 2006), but they still lacked a clear spectroscopic detection of an underlying supernova. Detection of such signature came with the discovery by the HETE-2 mission of GRB 030329 (Stanek et al. 2003 Hjorth et al. 2003). Due to its extreme brightness and slow decay, spectroscopic observations were extensive. The early spectra consisted of a power-law decay continuum $\left(F_{\nu} \propto \nu^{-0.9}\right)$ typical of GRB afterglows with narrow emission

\footnotetext{
${ }^{1}$ We note, however, that the first optical afterglow detection of GRB 970228 van Paradijs et al. 1997) was based on the X-ray prompt detection by BeppoSAX.
} 
features identifiable as $\mathrm{H} \alpha$, [OIII], $\mathrm{H} \beta$ and [OII] at $z=0.1687$ (Kawabata et al. 2003, Matheson et al. 2003) making GRB 030329 the second nearest burst overall at the time and the nearest classical burst ${ }^{2}$, A major contribution to our understanding of X-ray prompt emission came also from the HETE-2 mission (Lamb et al. 2004) which was active from 2000 to 2006. Dozens of bursts in the "X-ray flash" category were observed and were found to be similar in origin to the classical long GRBs (Matsuoka et al. 2004).

Swift is the current GRB discovery mission. It is a space robot designed specifically with GRBs in mind. It combines a wide-field hard X-ray burst detection telescope (Burst Alert Telescope - BAT; Barthelmy et al. 2005a), with narrowfield X-ray (X-Ray Telescope - XRT; Burrows et al. 2005a), and UV-optical (UV Optical Telescope - UVOT; Roming et al. 2005) telescopes. A powerful and fast on-board burst detection algorithm (Fenimore et al. 2003) provides the burst coordinates to the spacecraft, which autonomously repoints the observatory so that $\mathrm{X}$-ray and optical observations typically commence within two minutes of the burst trigger. The mission was designed to find counterparts for all burst types, including the previously-elusive short GRBs. Burst positions and other data are provided promptly to the ground for ground observers.

The burst detection rate for Swift is about 100 GRBs per year, resulting in a current data set as of December 2008 of 380 bursts. Of these, there are 126 with redshift determination, mostly from spectrographs on large optical telescopes and new robotic telescopes on the ground. These now far outnumber the $\sim 40$ GRB redshifts available prior to Swift. More than $95 \%$ of the Swift bursts have X-ray afterglow detection and $\sim 60 \%$ have optical afterglows (UVOT + ground). To date 33 short bursts have been localized with 8 having redshift determinations. The new data have enabled much more detailed studies of the burst environment, the host galaxy, and the intergalactic medium between galaxies. Swift and followup observations have also transformed our view of GRB sources. For example, as discussed in Section 3 , the old concept of a sudden release of energy concentrated in a few brief seconds has been discarded. Indeed, even the term "afterglow" is now recognized as misleading - the energy radiated during both phases is comparable.

Our primary intention in this review is to describe the most important observational discoveries in the Swift era as well as to explain some of the new understanding of key mechanisms that are believed to operate in these objects to an astronomical audience with little prior exposure to GRBs. Four sections follow. Section 2 provides a description of our current knowledge of what constitutes a GRB. Section 3 is a summary of the observations of the prompt and afterglow emission and Section IV of the observations of host galaxies and progenitor clues. In Section V, we examine our current progress in our understanding of the basic physical processes at work. Section VI is a look forward at future prospect for GRB study.

\footnotetext{
${ }^{2}$ The other GRBs with supernova associations have been underluminous events (Kaneko et al. 2007).
} 


\section{WHAT IS A GAMMA-RAY BURST?}

GRBs are sudden, intense flashes of $\gamma$-rays which, for a few blinding seconds, light up an otherwise fairly faint $\gamma$-ray sky. Spectra extending over many decades in photon energy have now been measured for hundreds of GRBs. In Figure 1 representative spectra are plotted in the conventional coordinates $\nu$ and $\nu F_{\nu}$, the energy radiated per logarithmic (natural log) frequency interval. Some obvious points should be emphasized. First, we measure directly only the energy radiated in the direction of the earth per second per steradian per logarithmic frequency interval by a source. The apparent bolometric luminosity may be quite different from the true bolometric luminosity if the source is not emitting isotropically. Second, there is striking evidence for a characteristic photon energy (peak in the $\nu F_{\nu}$ spectrum), which appears to be related to the overall spectral luminosity normalization. In contrast, the spectra of many galactic and extragalactic accretion systems are often well fitted by single power-laws. A simple powerlaw contains little information, whereas a complex spectrum composed of many broken power-laws tell us much more, as each break frequency must be explained.

At cosmological distances the observed GRB fluxes imply energies that can exceed $10^{53}(\Omega / 4 \pi)$ erg, where $\Omega$ is the solid angle of the emitting region (Figure 2 , see also Bloom et al. 2001). This is the mass equivalent of $0.06 M_{\odot}$ for the isotropic case. Compared with the size of the sun, the seat of this activity is extraordinarily compact, with sizes of less than milli-light-seconds $(<300 \mathrm{~km})$ as indicated by rapid variability of the radiation flux (Bhat et al. 1992). It is unlikely that mass can be converted into energy with better than a few (up to ten) percent efficiency; therefore, the more powerful GRB sources must "process" upwards of $10^{-1}(\Omega / 4 \pi) M_{\odot}$ through a region which is not much larger than the size of a neutron star (NS) or a stellar mass black hole (BH). No other entity can convert mass to energy with such a high efficiency, or within such a small volume.

The observed $\gamma$-rays have a nonthermal spectrum. Moreover, they commonly extend to energies above $1 \mathrm{MeV}$, the pair production threshold in the rest frame. These facts together imply that the emitting region must be relativistically expanding (Guilbert et al. 1983, Goodman 1986, Paczyński 1986). We draw this conclusion for two reasons. First, if the region were indeed only a light second across or less, as would be implied by the observed rapid variability in the absence of relativistic effects, the total mass of baryons in the region would need to be below about $10^{-12} M_{\odot}$ in order that the electrons associated with the baryons should not provide a large opacity (Piran and Shemi 1993, Paczyński 1990). Second, larger source dimensions are required in order to avoid opacity due to photon-photon collisions. If the emitting region is expanding relativistically, then, for a given observed variation timescale, the dimension $R$ can be increased by $\Gamma^{2}$. The opacity to electrons and pairs is then reduced by $\Gamma^{4}$, and the threshold for pair production, in the observer frame, goes up by $\sim \Gamma$ from its rest frame value (Fenimore et al. 1993, Woods and Loeb 1995, Baring and Harding 1997, Granot et al. 2008). Best-guess numbers are Lorentz factors $\Gamma$ in the range $10^{2}$ to $10^{3}$ 


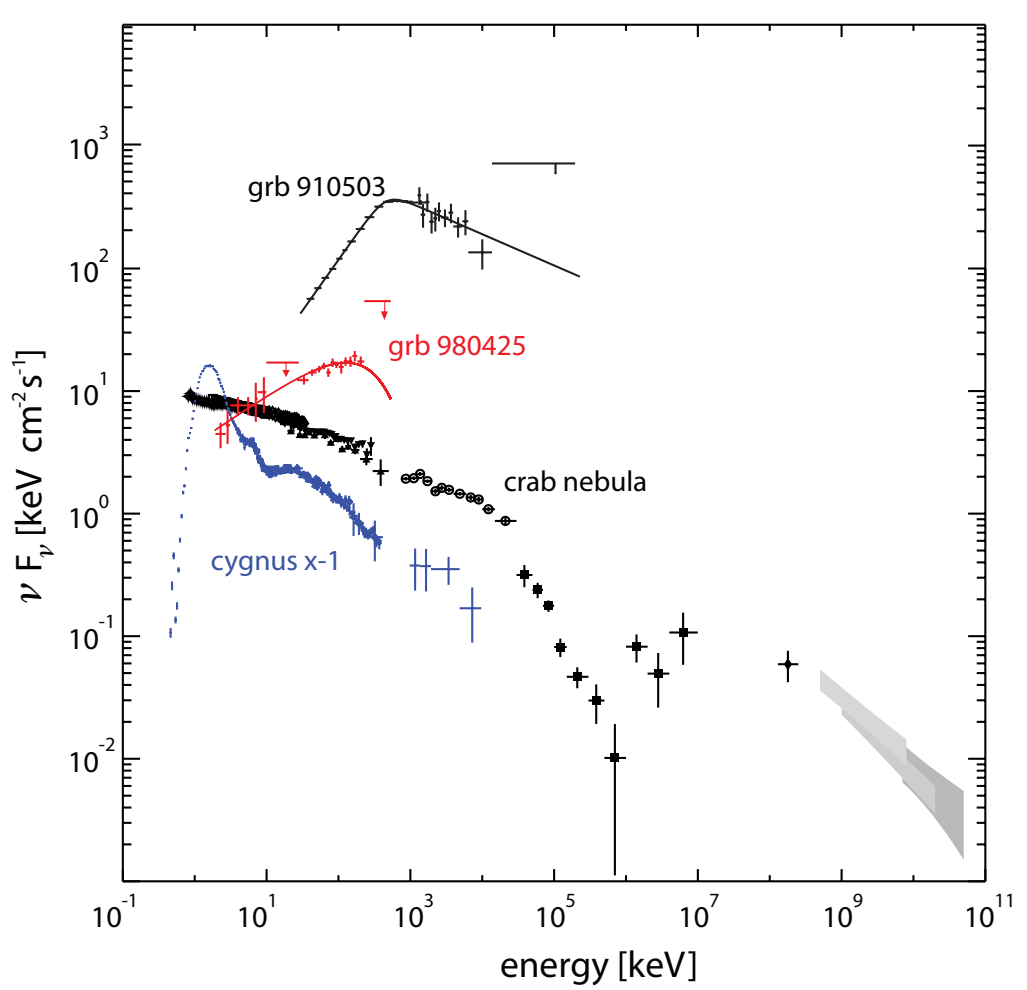

Figure 1 Gamma-rays are excellent probes of the most energetic phenomena in nature, that typically involve dynamical non-thermal processes and include interactions of high energy electrons with matter, photons and magnetic fields; high energy nuclear interactions; matter- antimatter annihilation and possibly other fundamental particle interactions. Here shown are representative spectra $\nu F_{\nu} \propto \nu^{2} N(\nu)$ of GRBs (Kaneko et al. 2007: Kaneko et al. 2008) together with the Crab pulsar nebula (Kuiper et al. 2001) and the galactic black hole candidate Cygnus X-1 (McConnell et al. 2002).

(Lithwick and Sari 2001), allowing rapidly-variable emission to occur at radii in the range $10^{12}$ to $10^{14} \mathrm{~cm}$.

Because the emitting region must be several powers of ten larger than the compact object that acts as a trigger, there are further physical requirements. The original internal energy contained in the radiation and pairs would, after expansion, be transformed into relativistic kinetic energy. A variant that has also been suggested is based on the possibility that a fraction of the energy is carried by Poynting flux (Blandford and Znajek 1977, Usov 1992). This energy cannot be efficiently radiated as $\gamma$-rays unless it is re-randomized (Mészáros et al. 1994. Narayan et al. 1992, Rees and Mészáros 1994, Paczyński and Xu 1994). Impact on an external medium (or an intense external radiation field; e.g., Shaviv and Dar 1995) would randomize half of the initial energy merely by reducing the expansion Lorentz factor by a factor of 2 . For an approximately smooth distribution of external matter, the bulk Lorentz factor of the fireball thereafter decreases as an inverse power of the time. In the presence of turbulent magnetic fields built 


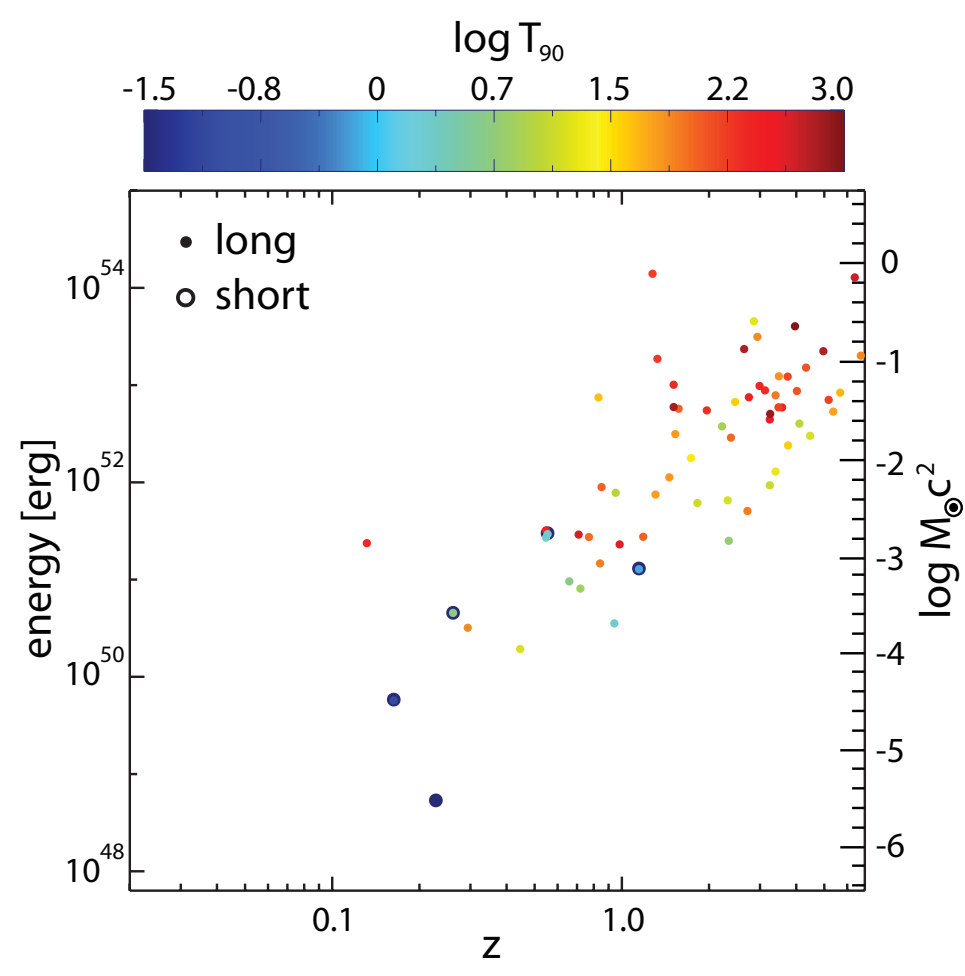

Figure 2 Apparent isotropic $\gamma$-ray energy as a function of redshift and observed duration. The energy is calculated assuming isotropic emission in a common comoving bandpass for a sample of short and long GRBs with measured redshifts. This spread in the inferred luminosities obtained under the assumption of isotropic emission may be reduced if most GRB outflows are jet-like. A beamed jet would alleviate the energy requirements, and some observational evidence does suggest the presence of a jet.

up behind the shocks (Rees and Mészáros 1992), the electrons produce a synchrotron power-law radiation spectrum which softens in time, as the synchrotron peak corresponding to the minimum Lorentz factor and field decreases during the deceleration (Katz 1994, Sari et al. 1996). Thus, the GRB radiation, which started out concentrated in the $\gamma$-ray range during the burst, is expected to progressively evolve into an afterglow radiation which peaks in the X-rays, then UV, optical, IR and radio (Figure 3). Detailed predictions (Mészáros and Rees 1997) of the afterglow properties, made in advance of the observations, agreed well with subsequent detections at these photon energies, followed up over periods of up to months. The detection of diffractive scintillation in the radio afterglow of GRB 970508, provided the first determination of the source size and a direct confirmation of relativistic source expansion (Frail et al. 1997), which was further strengthened by the size measurement of the afterglow image of GRB 030329 by radio interferometry with the VLBA (Taylor et al. 2004).

The complex time-structure of some bursts suggests that the central engine may remain active for up to $100 \mathrm{~s}$ (Ramirez-Ruiz and Fenimore 2000) or possibly longer (Falcone et al. 2007). However, at much later times all memory of the 

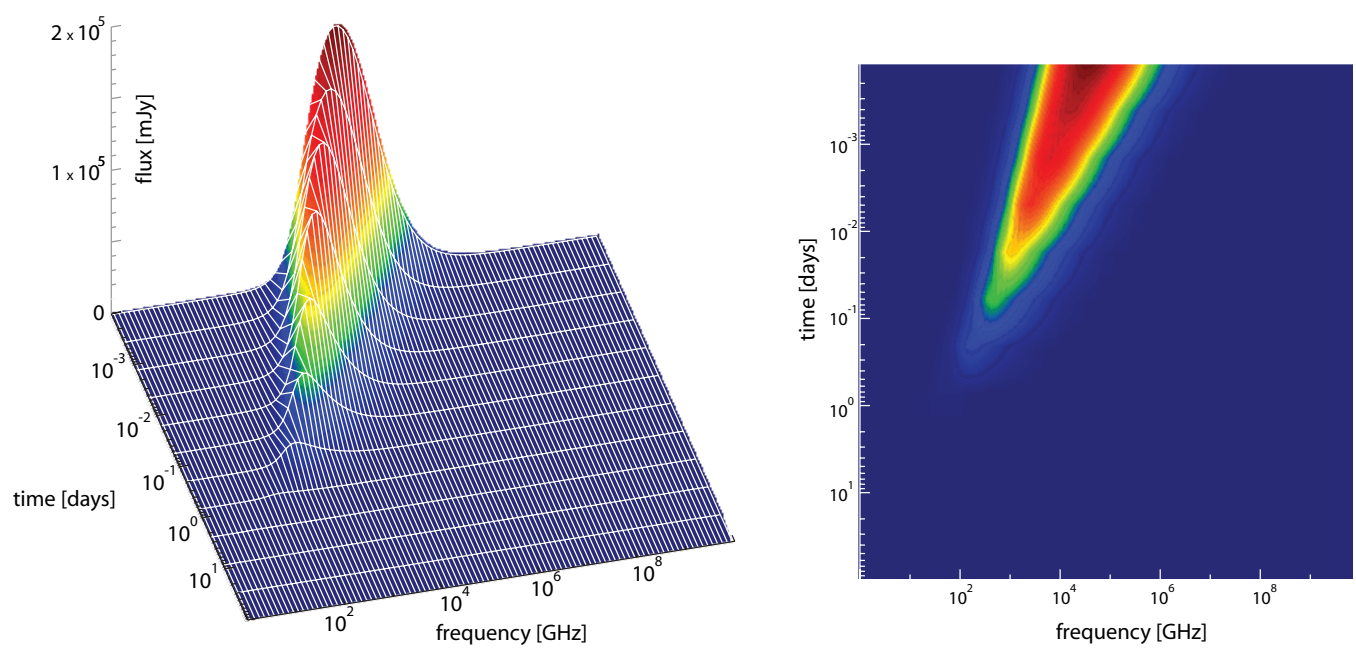

Figure 3 The evolving synchrotron afterglow of a $\gamma$-ray burst. Shown is a theoretical model (Gou et al. 2007) for the afterglow of the Swift GRB 050904. The model is presented without extinction and as it would have been observed at redshift $z=2$; the burst itself occurred at $z=6.29$. The evolution of the synchrotron peak to lower frequencies is clearly visible. More subtle effects including evolution of the synchrotron cooling and self-absorption frequencies, and the associated synchrotron self-Compton emission of the blastwave at higher frequencies, are not readily visible in this model.

initial time-structure would be lost: essentially all that matters is how much energy and momentum has been injected, its distribution in angle and velocity. We can at present only infer the energy per solid angle, but there are reasons to suspect that bursts are far from isotropic. Due to relativistic beaming, an observer will receive most emission from those portions of a GRB blast wave that are within an angle $\sim 1 / \Gamma$ of the direction to the observer. The afterglow is thus a signature of the geometry of the ejecta - at late stages, if the outflow is beamed, we expect a spherically-symmetric assumption to be inadequate; the deviations from the predictions of such a model would then tell us about the ejection in directions away from our line of sight (Rhoads 1999).

The appearance of achromatic breaks in the development of GRB afterglows has been interpreted as indicating that they are jet flows beamed towards us. Collimation factors of $\Omega_{i} / 4 \pi \lesssim 0.01$ (corresponding to half opening angles of $\lesssim 8$ degrees) have been derived from such steepening (Frail et al. 2001, Bloom et al. 2003). If GRB sources are beamed, then this reduces the energy per burst by two or three orders of magnitude at the expense of increasing their overall frequency.

As regards the central engine trigger, there remain a number of key questions. What are the progenitors? What is the nature of the triggering mechanism, the transport of the energy and the time scales involved? Does it involve a hyperaccreting compact object? If so, can we decide between the various alternative ways of forming it? The presence of some GRBs (in the short duration category) 
in old stellar populations rules out a source uniquely associated with recent star formation and, in particular, massive star origin for all bursts (Gehrels et al. 2005, Bloom et al. 2006). An understanding of the nature of these sources is thus inextricably linked to the metabolic pathways through which gravity, spin, and energy can combine to form collimated, ultrarelativistic outflows. These threads are few and fragile, and the tapestry is as yet a poor image of the real universe. If we are to improve our picture-making we must make more and stronger ties to physical theory. But in reconstructing the creature, we must be guided by our

eyes and their extensions. The following sections provide a detail summary of the observed properties of these ultra-energetic phenomena. These threads will be woven in Section 5 .

\section{BURST AND AFTERGLOW OBSERVATIONS}

The most direct diagnostics of the conditions within GRBs come from the radiation we observe, which we summarize in this section. There are many ways to organize a discussion of the properties of the radiation emanating from GRB sources. We do not intend in this article to a give a detailed review of individual events since there are now sufficiently many examples that we are likely to be led seriously astray if we test our theories against individual events. For this reason, we center our discussion on major trends even in cases in which the generalizations we describe are based on data that do not yet have the statistical weight of a "complete" sample. It should be noted that there are inherent biases in the discovery of a GRB at a given redshift which are often difficult to quantify, such as complex trigger efficiencies and non-detections. Continued advances in the observations will surely yield unexpected revisions and additions in our understanding of the properties of GRBs.

\subsection{Prompt High-Energy Emission}

3.1.1 Taxonomy. The manifestations of GRB activity are extremely diverse. GRBs are observed throughout the electromagnetic spectrum, from $\mathrm{GHz}$ radio waves to $\mathrm{GeV} \gamma$-rays, but until recently, they were known predominantly as bursts of $\gamma$-rays, largely devoid of any observable traces at any other wavelengths. $\gamma$-ray properties provide only one of several criteria for classifying GRB sources. Part of the problem is observational because it is not possible to obtain full spectral coverage in all objects and it is not easy to reconcile a classification based on host galaxy properties with one based on the prompt $\gamma$-ray properties. The major impediment to serious taxonomy is more fundamental. GRBs are heterogeneous objects, especially in their directly observed properties. The success of a classification scheme, we believe, should be measured by the extent to which newly recognized properties distinguish subsets defined by differences in other properties. By this criterion, the taxonomy of GRBs has met with only mixed success. As new non- $\gamma$-ray selection techniques are introduced (e.g. age of stellar populations in host galaxies or the presence of type Ic supernova signatures), the class boundaries (e.g. short and long duration events) have blurred where the 
defined subclasses transcend traditional boundaries. On the other hand, many new properties do correlate with old ones. This is all the more remarkable in that the conventional diagnostics (e.g. burst duration) measure properties on scales several orders of magnitude larger than that which we believe to be the characteristic of the powerhouse.

3.1.2 Observed durations and redshifts. GRB traditionally have been assigned to different classes based on their duration - usually defined by the time during which the middle $50 \%\left(T_{50}\right)$ or $90 \%\left(T_{90}\right)$ of the counts above background are measured. On the basis of this criterion, there are two classes of GRBs short and long - where $\sim 2 \mathrm{~s}$ duration separates them. The initial hints for the existence of such classes (Cline and Desai 1974, Mazets et al.1981), were followed by stronger evidence from ISEE-3 and Konus-WIND data (Norris et al. 1984) and definite proof using large statistics from BATSE (Kouveliotou et al. 1993). BATSE results also showed that short bursts have a harder spectrum than long bursts (Kouveliotou et al. 1993) although this is less prominent in observations by Konus-WIND, HETE-2, and Swift (Sakamoto et al. 2006).

The duration and redshift distributions for Swift GRBs are shown in Figure 4. The blue histogram in the left panel is for observed durations while the orange histogram shows the durations corrected to the source frame $T_{90} /(1+z)$ for those bursts with redshift determinations. In the source frame, the typical long burst duration is $\sim 20$ s compared to $\sim 50$ s in the observer frame. Swift has been detecting a lower fraction $(\sim 10 \%)$ of short bursts than BATSE did $(25 \%)$. This is because Swift observes in a softer energy band $(15-150 \mathrm{keV})$ than BATSE $(50 \mathrm{keV}-2 \mathrm{MeV})$ and because Swift requires a sky image of the event for burst detection and the image part of the trigger algorithm is less sensitive to short bursts due to their lower fluences. Shown in Figure 4 (right panel) are the measured redshift distributions. The blue histogram is for Swift events while the gray one is for pre-Swift bursts. As clearly seen, Swift is currently detecting GRBs at a higher average redshift: $\langle z>\sim 2.5$ for Swift bursts while $\langle z>\sim 1.2$ for pre-Swift events. The reason for this difference is the higher sensitivity of Swift compared to BeppoSAX and HETE-2.

GRBs have also been classified according to their spectral properties, albeit less successfully. In particular, bursts with lower spectral energy peaks $\left(E_{\text {peak }}\right)$ have been denoted X-Ray Flashes (XRFs) based on observations by BeppoSAX, BATSE and HETE-2 (Heise et al. 2001, Barraud et al. 2003, Kippen et al. 2003, Sakamoto et al. 2005). These events are closely related to common long duration GRBs and appear to form a continuum of all parameters between the two types with no striking evidence for a discerning characteristic (Granot et al. 2005b).

3.1.3 Observed correlations. There is a great deal of diversity in the $\gamma$-ray prompt lightcurves of GRBs. Both long and short bursts can have temporal profiles ranging from smooth, single-peaked pulses to highly structured multi-pulses. The prompt emission can be characterized by a variety of spectral and temporal parameters which include duration, variability, lag, pulse rise/fall time, fluence, 

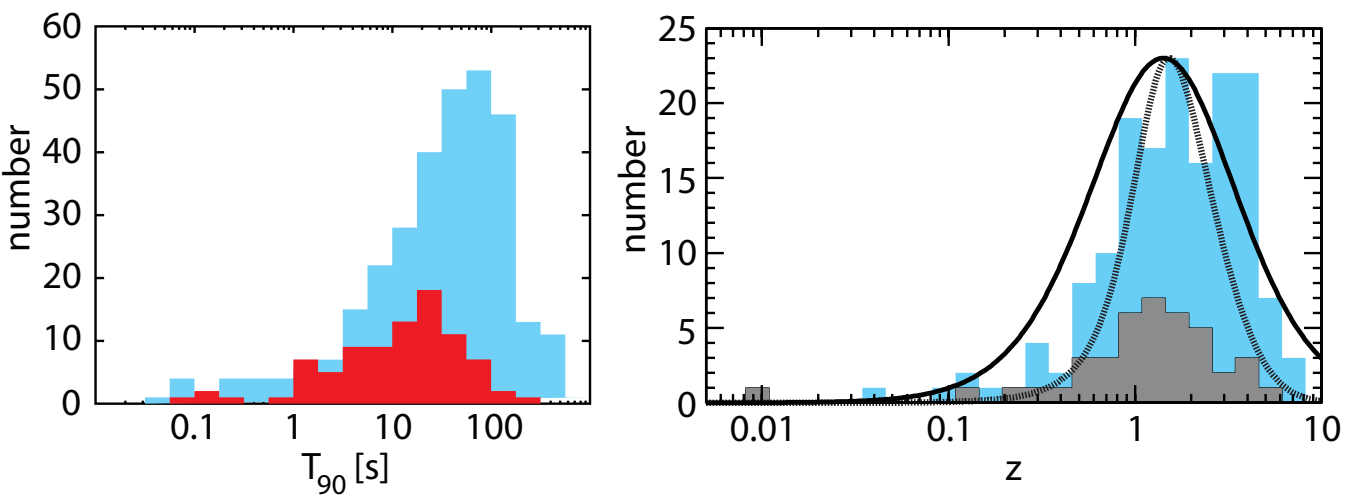

Figure 4 Duration and redshift distributions for Swift GRBs. Left panel shows the duration distribution. The blue histogram is the measured $T_{90}$ distribution while the orange one is corrected to the source frame: $T_{90} /(1+z)$. Right panel shows the redshift distribution for Swift GRBs in blue and pre-Swift GRBs in gray. Swift is detecting higher redshift bursts on average than pre-Swift. The solid (broad) theory curve illustrates the evolution of a comoving volume element of the universe while the dotted (narrow) curve is a convolution of the comoving volume with a model for the star formation rate as calculated by Porciani and Madau (2001).

$E_{\text {iso }}$ and $E_{\text {peak }}$. A schematic diagram illustrating the most widely discussed $\gamma$ ray prompt correlations is shown in Figure 5 with detailed references given in its caption. These correlations are often based upon statistical analysis of quantities whose physical causes are poorly understood, but almost certainly depend on many variables. Interpretations of these correlations must therefore be done with caution.

The prompt GRB lightcurves can generally be dissembled into a superposition of individual pulses as described by Norris et al. (1996) with rise times shorter on average than decay times ( panel $f$ ). The variability or spikeness of the lightcurve is found to be correlated with peak luminosity or total isotropic energy of the burst (panel a). The time lag of individual peaks seen at different energy bands is observed to be anticorrelated with luminosity for long bursts (panel b). For short bursts, the lag is small or not measurable. The $E_{\text {peak }}$ is also found to be correlated with $E_{\text {iso }}$ for long bursts including XRFs with short bursts as clear outliers ( panel c). The total isotropic energy emission is correlated with duration (panel d), with short and long bursts on approximately the same correlation line, albeit with a wide spread. Short bursts detected by Swift have lower $E_{\text {iso }}$ on average than long bursts. There is a group of outliers belonging to the long burst category which are characterized by being significantly underluminous. These are GRBs: 980425, 031203, 060614.

Numerous researchers have studied ways to determine the absolute luminosity of a GRB using correlations such as those illustrated in Figure 5. These include the lag, variability and $E_{\text {peak }}$ correlations discussed above. Other interesting correlations have included $E_{\text {peak }}$ versus $E_{\gamma}$ (emitted energy corrected for beaming, Ghirlanda et al. 2004) and $E_{\text {peak }}$ versus a duration corrected peak luminosity 
(Firmani et al. 2006). The goal is to derive a method to determine the burst luminosity independently of a redshift distance determination, thus attempting to make GRBs standard candles which could be used, in principle, to determine the cosmic expansion history of the universe to higher redshift than with supernovae. Although such efforts are currently underway (Schaefer 2006, and references therein), it is not clear at present whether any of these correlations are tight enough for significant progress to be made (Bloom et al. 2003).

3.1.4 Soft $\gamma$ repeaters and short bursts. It has been noted (Hurley et al. 2005, Palmer et al. 2005, Nakar et al. 2006b) that the giant flare (GF) observed from the putative galactic magnetar source SGR1806-20 in December 2004 (Gaensler et al. 2005) could have looked like a typical short GRB had it occurred much farther away, thus making the tell-tale periodic signal characteristic of the neutron star rotation in the fading emission undetectable. The two previously recorded GFs of this type, one each from SGR 0520-66 on 5 March 1979 (Fenimore et al. 1996a) and SGR 1900+14 on 27 August 1998 (Hurley et al. 1999), would have been detectable by existing instruments only out to $\sim 8 \mathrm{Mpc}$, and it was therefore not previously thought that they could be the source of short GRBs. The main spike of the 27 December event would have resembled a short, hard GRB if it had occurred within $\sim 40 \mathrm{Mpc}$, a distance scale encompassing the Virgo cluster (Palmer et al. 2005). However, the paucity of observed GFs in our own Galaxy has so far precluded observationally based determinations of either their luminosity function or their rate. The observed isotropic distribution of short BATSE GRBs on the sky and the lack of excess events from the direction of the Virgo cluster suggests that only a small fraction, $\leq 5 \%$, of these events can be SGR GFs within 40 Mpc (Palmer et al. 2005).

Before Swift detected short GRBs and their associated afterglow signatures, searches for nearby galaxies within narrow Inter Planetary network (IPN) error boxes revealed that only up to $\simeq 15 \%$ of them could be accounted for by SGRs capable of producing GFs (Nakar et al. 2006b). A recent, intriguing candidate is short GRB 070201 which was observed by the IPN to have a location consistent with the arms of the nearby $(0.8 \mathrm{Mpc})$ M31 galaxy (Mazets et al. 2008). A LIGO search for gravitational waves (Abbott et al. 2008) at the time of the burst turned up with no signal, thus excluding a compact merger origin. If the GRB was really in M31, it may have been an SGR GF. While the fraction of SGR events among what are now classified as short GRBs may not be dominant, it should be detectable and can be tested with future Swift observations. It is also worth noting that some short GRBs likely originate in the local univserse (Tanvir et al. 2005).

\subsection{Afterglow Observations}

3.2.1 X-ray observations. Swift was designed to investigate the GRB afterglows by filling the temporal gap between observations of the prompt emission and the afterglow. The combined power of the BAT and XRT has revealed that prompt X-ray emission smoothly transitions into the decaying afterglow 
a)

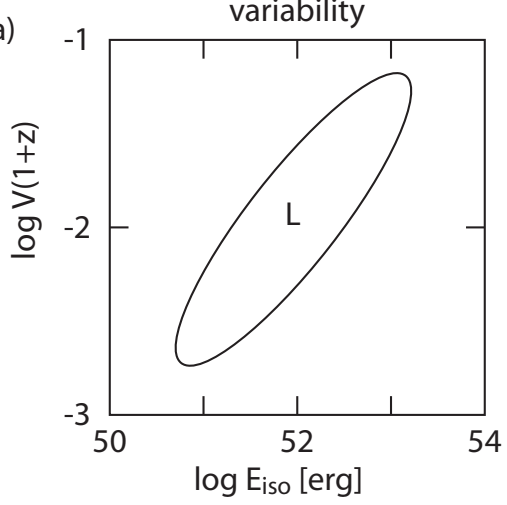

c)

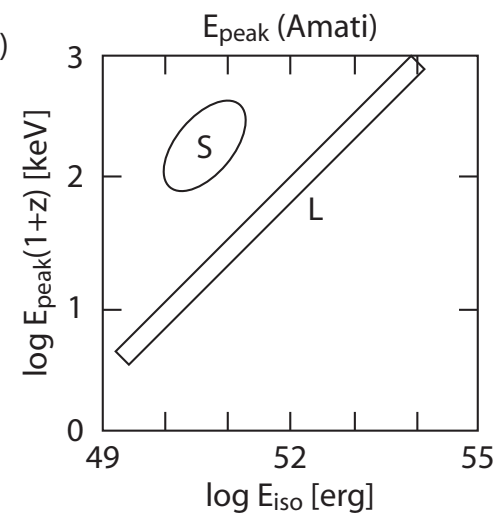

e)

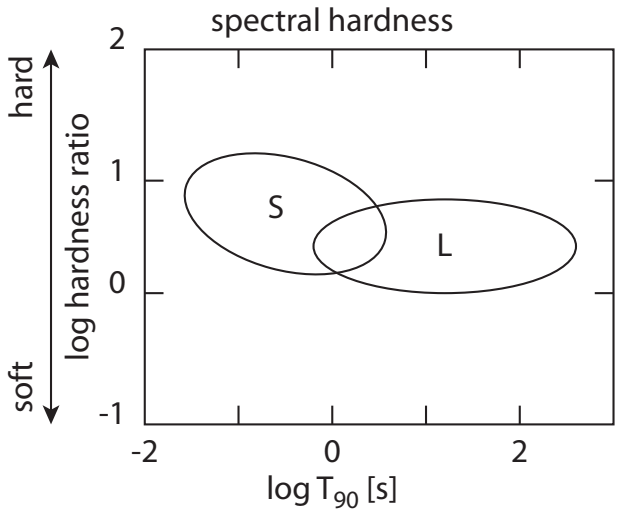

b)

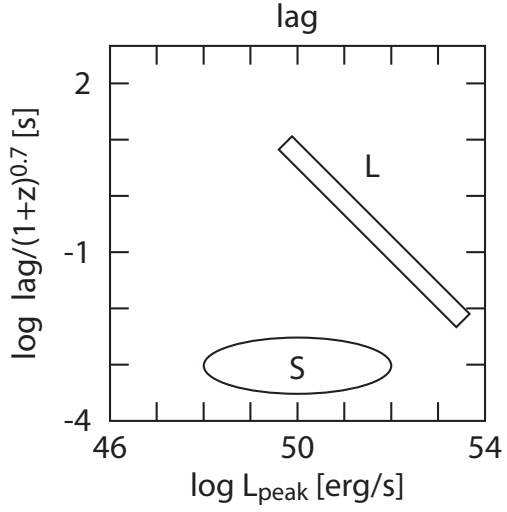

d)

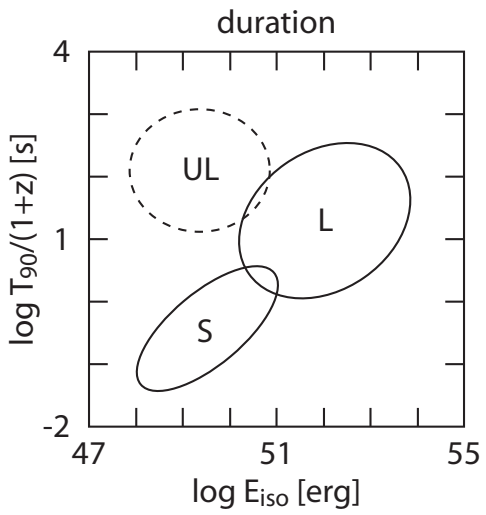

f)

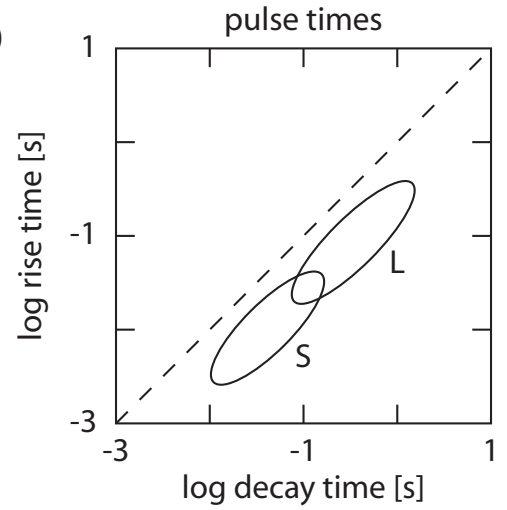

Figure 5 Schematic diagrams illustrating the most widely discussed correlations between various prompt emission properties for long (L), short (S), and underluminous (UL) GRBs. (a) Variability scaled to the burst frame versus $E_{\text {iso }}$ (Fenimore and Ramirez-Ruiz 2000, Reichart et al. 2001, Schaefer 2006). The variability is a measure of the spikiness of the lightcurve and is defined as the mean square of the time signal after removing low frequencies by smoothing. (b) Spectral lag scaled to the burst frame versus peak luminosity (Norris and Bonnell 2006, Gehrels et al. 2006). (c) $E_{\text {peak }}$ scaled to the burst frame versus $E_{\text {iso }}$ (Amati et al. 2002 for BeppoSAX GRBs; Amati 2006 for Swift GRBs; Lloyd-Ronning and Ramirez-Ruiz 2002 for BATSE events). (d) Duration scaled to the burst frame versus $E_{\text {iso. }}$ (e) Spectral hardness versus observed duration (Kouveliotou et al. 1993). (f) Pulse rise time versus its decay time (Norris et al. 1996). 

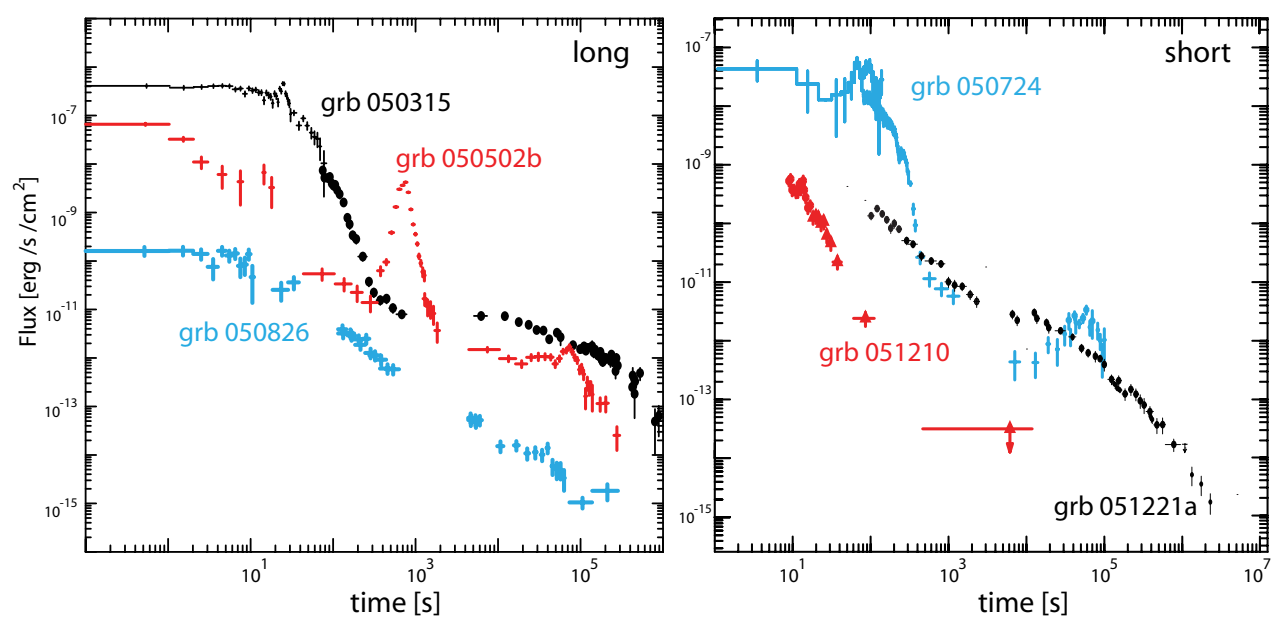

Figure 6 Representative examples of X-ray afterglows of long and short Swift events with steep-to-shallow transitions (GRB 050315, 050724), large X-ray flares (GRB 050502B, 050724), fastly declining (GRB 051210) and gradually declining (GRB 051221a, GRB 050826; flux scale divided by 100 for clarity) afterglows.

(Barthelmy et al. 2005b, O'Brien et al. 2006). Three representative Swift X-ray lightcurves are shown in Figure 6 for both long and short GRBs. These X-ray light curves start as early as a hundred seconds after the GRB trigger, and cover up to five decades in time. The complex behavior revealed in them sigificantly challenges traditional afterglow theoy, and calls into question some of the basic underlying assumptions.

One of most striking results is that many of the early X-ray afterglows show a canonical behavior, where the light curve broadly consists of three distinct power law segments (Nousek et al. 2006). A bright rapid-falling $\left(t^{-\alpha}\right.$ where $\alpha>3$ ) afterglow immediately after the prompt emission (Tagliaferri et al. 2005b) is followed by a steep-to-shallow transition, which is usually accompanied by a change in the spectrum power-law index. This is consistent with an interpretation (Nousek et al. 2006, Zhang et al. 2006) in which the first break occurs when the slowly decaying emission from the forward shock becomes dominant over the steeply decaying tail emission of the prompt $\gamma$-rays as seen from large angles (Kumar and Panaitescu 2000). Since these two components arise from physically distinct regions, their spectrum would generally be different. The shallow phase then transitions to the classical afterglow phase with no clear evidence for a spectral change. In some cases a jet break is seen at late times. The intermediate shallow flux stage is commonly interpreted as being caused by the continuous energy injection into the external shock (Nousek et al. 2006, Zhang et al. 2006) although orientation and complex jet structures have been also discussed as viable alternatives. The energy in the afterglow at these late times is estimated to be comparable to or smaller than that in the prompt gamma-ray emission, even when correcting for radiative losses from the afterglow shock at early times, implying a high efficiency of the prompt emission. The presence of the shallow decay phase 
implies that most of the energy in the afterglow shock was either injected at late times after the prompt gamma-ray emission was over or was originally in slow material that would not have contributed to the prompt gamma-ray emission. This requires the prompt gamma-ray emission mechanism to be significantly more efficient than previous estimates. If a significant fraction of the radiated energy goes to photon energies above the observed range, the efficiency requirements of the prompt emission become even more severe.

Table 1 Typical parameters of the canonical Swift X-ray light curve

\begin{tabular}{lccc}
\hline Phase & Start T (s) & Decay index $^{a}$ & $\begin{array}{c}\text { Approximate } \\
\text { frequency }\end{array}$ \\
\hline Steep decline & $10^{1}-10^{2}$ & $>3$ & $50 \%$ \\
Shallow slope & $10^{2}-10^{3}$ & 0.5 & $60 \%$ \\
Classical afterglow & $10^{3}-10^{4}$ & 1.3 & $80 \%$ \\
Jet break late phase & $10^{5}-10^{6}$ & 2.3 & $20 \%^{b}$ \\
X-ray flares & $10^{2}-10^{4}$ & & $50 \%$ \\
\hline
\end{tabular}

${ }^{a}$ Decay index $\alpha$ defined by $F=F_{o} t^{-\alpha}$.

${ }^{b}$ Of the $80 \%$ with no observed jet break, about half had afterglow observations terminate before expected time of jet break.

The average times, slopes, and frequencies characterizing these three distinct X-ray afterglow components are listed in Table 1. Most Swift X-ray light curves are broadly consistent with this basic temporal description, although in most cases we do not see all three power law segments, either because not all are present or because of limited temporal coverage. The large variety of behaviors exhibited by afterglows at different times in their evolution can be seen in Figure 7, which shows the temporal history for each individual afterglow as well as the evolution of the cumulative X-ray afterglow luminosity for a large sample of Swift events with known redshift. While broadly compatible with relativistic fireball models (Nousek et al. 2006, Zhang et al. 2006) the complex afterglow behavior that has been revealed poses new challenges of interpretation. The reader is refered to Granot (2008) for a more detailed account of the major strengths and weaknesses of the standard afterglow model, as well as some of the challenges that it faces in explaining recent data.

Swift has also discovered flaring behavior appearing well after the prompt phase in $\sim 50 \%$ of X-ray afterglows (Chincarini et al. 2007, Falcone et al. 2007). An illustration of bursts with bright flares is shown in Figure 6. In some extreme cases, the late time flares have integrated energy similar to or exceeding the initial burst of $\gamma$-rays (Burrows et al. 2005b). The rapid rise and decay, multiple flares in the same burst, and cases of fluence comparable to the prompt emission suggest that these flares are due to the same mechanism responsible for the prompt emission, which is usually attributed to the activity of the central powerhouse. When X-ray flares are observed by XRT, it is typically the case that no flaring is seen in the optical band by the UVOT. A notable example is GRB 060418 (Molinari et al. 2007), whose optical-infrared afterglow spectra is not consistent with 


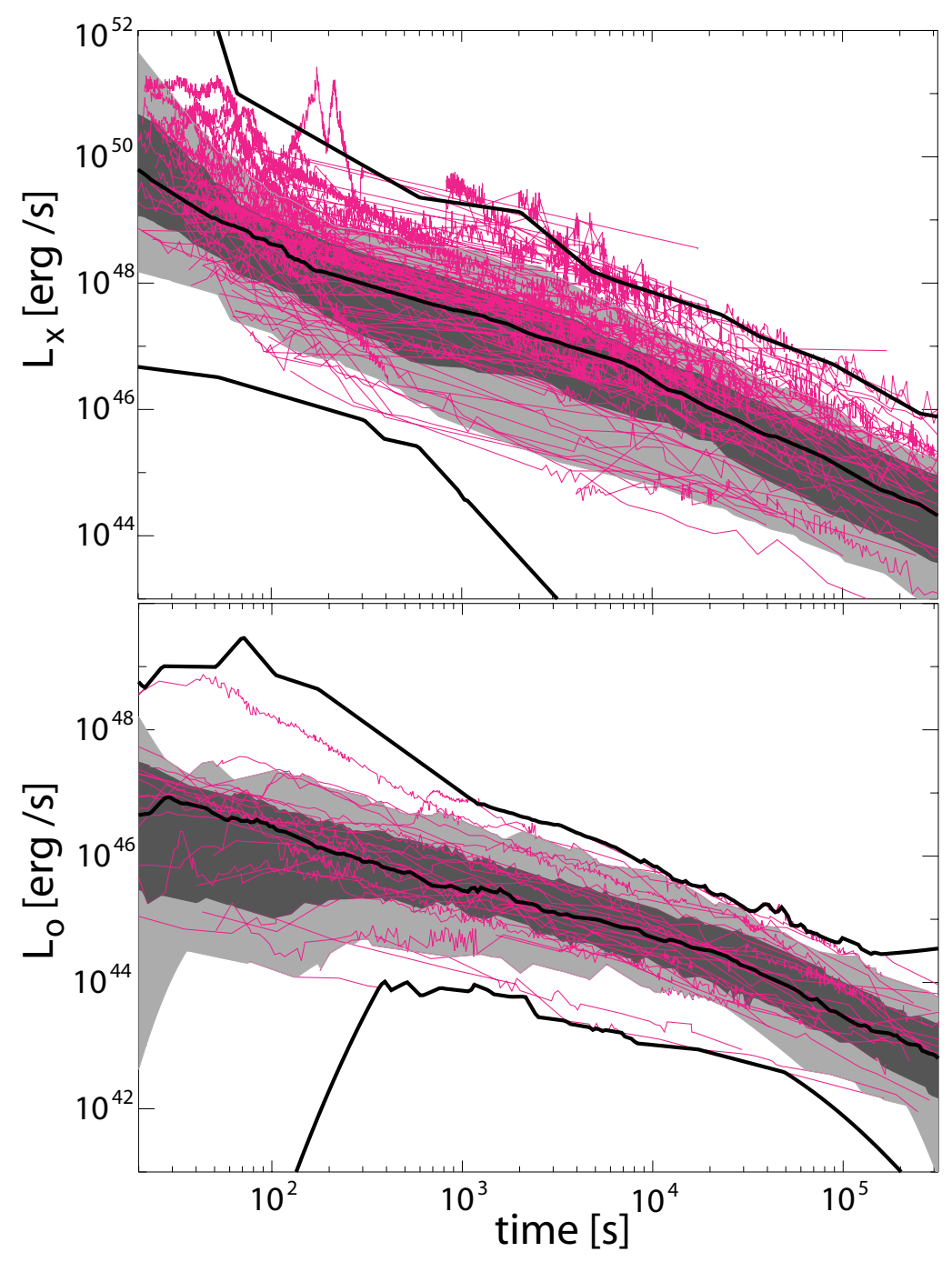

Figure 7 X-ray and optical lightcurves of GRB afterglows in the Swift era. (a) X-ray lightcurves of Swift burst afterglows. Data for long-duration bursts with known redshifts, from GRB 050126 to GRB 070724A, have been gathered from the Swift XRT lightcurve and spectral data depository at the UK Swift Science Data Centre (Evans et al. 2007; Evans et al. 2008). They are corrected uniformly to unabsorbed luminosity over $1.0-30.0 \mathrm{keV}$ in the burst rest-frame, using the time-average afterglow spectrum, and plotted as a function of rest-frame time (pink lines). Separately, afterglow lightcurve fits (Racusin et al. 2008b), which exclude flaring intervals, are used to construct minimum and maximum envelopes (black lines) and confidence intervals (gray bands) on the X-ray luminosities of the bursts as a function of rest-frame time: light gray regions delimit bands of $10 \%$ to $90 \%$ confidence, dark gray regions delimit bands of $25 \%$ to $75 \%$ confidence, and the median burst luminosity at any given time is shown (middle black line). (b) Optical lightcurves of Swift burst afterglows. Data for long-duration bursts with known redshifts and at least "bronze" quality published optical data (Kann et al. 2007), from GRB 050408 to GRB $070612 \mathrm{~A}$, are corrected uniformly to rest-frame $U$-band luminosity using the inferred $R$-band $(z=1)$ light-curves from Kann et al. (2007), and plotted as a function of rest-frame time (pink lines). Interpolated and "best fit" extrapolated lightcurves are used to generate minimum and maximum envelopes and median luminosity estimates (black lines) and confidence intervals (gray regions), as in $a$. 
a simple power-law extrapolation to soft X-ray energies and clearly requires two distinct spectral components. Not surprisingly, the broadband energy spectra of GRBs are complex and have spectra of wildly disparate shapes even for members belonging to a single class.

Prior to Swift there were several reports of emission and absorption line observations in the X-ray spectra of GRB afterglows. These included BeppoSAX observations of GRB 970508 (Piro et al. 1999) and GRB 000214 (Antonelli et al. 2000), ASCA observations of GRB 970828 (Yoshida et al. 1999), Chandra observations of GRB 991216 (Piro et al. 2000) and XMM observations of GRB 011211 (Reeves et al. 2002). None of the detections were of high statistical significance, but, combined, were of some credibility. Swift has not found any significant line features in comprehensive observations of the X-ray afterglow of more than 200 GRBs (Romano et al. 2008). Hurkett et al. (2008) also made a very detailed Swift X-ray line search, with no positive result. These strong negative findings call into question the significance of previous results.

A final, but widely discussed, observational development is the discovery in $\sim 25 \%$ of short bursts detected by Swift/BAT of an extended emission (EE) component lasting for $\sim 100$ seconds (Norris and Gehrels 2008). This component was clearly detected in HETE-2 burst GRB 050709 (Villasenor et al. 2005) and Swift burst GRB 050724 (Barthelmy et al. 2005c). Archival searches have also found BATSE bursts with EE (Norris and Bonnell 2006, Lazzati et al. 2001, Connaughton 2002). The EE is typically softer than the main peak and has an intensity range from $10^{-3}$ to $10^{-1}$ times that of the initial short pulse complex. It is possible that many of the $75 \%$ of bursts without currently detected EE have this component at flux levels below detectability, although there are bursts with upper limits on the intensity of $<10^{-4}$ times that of the short pulse complex.

3.2.2 Optical observations. With increasing frequency during the Swift era, optical observations of GRBs have been commencing almost immediately after - and in one noteworthy case (GRB 080319B; Racusin et al. 2008a), prior to - the GRB trigger itself. These early optical observations have substantially enriched our appreciation for the complexity of the physical processes active during the prompt emission phase of GRBs, and during the afterglow that follows. The large diversity of optical afterglow lightcurves can be seen in Figure 7 .

Prompt emission. Observations prior to the Swift era demonstrated already that bright optical flashes such as that seen from GRB 990123 Akerlof et al. 1999), were relatively rare (Kehoe et al. 2001). The success of the Swift mission has brought a vast increase in the rate of bursts accessible to rapid optical followup, and a corresponding increase in the number of events detected from early times, $t<100 \mathrm{~s}$. The Swift UVOT itself routinely detects optical afterglows following the intial prompt slew of the satellite, with a $40 \%$ detection rate for such bursts (Roming et al. 2009) that is only slightly lower than the $60 \%$ detection rate combining all observatories.

The fastest routine responses to Swift alerts are realized by robotic ground- 
based telescopes. The first discovery yielded by these observatories in the Swift era was of the $\gamma$-ray correlated component of the prompt optical emission (Vestrand et al. 2005, Blake et al. 2005, Vestrand et al. 2006). This component is not observed in every burst, but the mere fact of its correlation is sufficient to establish a common origin with the prompt $\gamma$-ray emission (e.g., internal shocks). When observed, the ratio of the correlated $\gamma$-ray to optical flux densities has been found to be roughly $10^{5}$ to one.

In contrast to bursts with $\gamma$-ray correlated emission, the common burst is now revealed to either exhibit a single power-law decay from early times (Rykoff et al. 2005b, Quimby et al. 2006a, Yost et al. 2006a), or to exhibit a flat or rising (Rykoff et al. 2004a, Rykoff et al. 2006a) or rebrightening (Stanek et al. 2007) optical light curve before it enters the standard power-law afterglow decay. The initial brightness of the typical counterpart is $V \sim 14$ to $17 \mathrm{mag}^{3}$ (Roming et al. 2009), which has made observations challenging for smaller $(<1 \mathrm{~m})$ robotic facilities, and has limited the extent of the lightcurves collected by the UVOT.

A few new observations of bright flaring optical emission have been collected, which are usually interpreted as emission from the reverse shock region (Sari and Piran 1999, Mészáros and Rees 1999). The early optical/NIR emission from GRB 041219 would have rivalled that seen from GRB 990123 if not for the large Galactic extinction along the line of sight (Vestrand et al. 2005). Of the three distinct peaks observed by PAIRITEL, the second may represent the onset of the afterglow (reverse shock) contribution (Blake et al. 2005) - if so, a relatively small Lorentz factor, $\Gamma \sim 70$, is derived by associating the flaring peak time with deceleration time of the relativistic blast wave. Observations of GRB 050525A with UVOT (Blustin et al. 2006) and GRB 060111B with TAROT (in a unique time-resolved tracking mode; Klotz et al. 2006) show the "flattening" light-curve familiar from GRB 021211 (Zhang et al. 2003). Intriguingly, the "high redshift" GRB 050904 (Tagliaferri et al. 2005a, Cusumano et al. 2006) at $z=6.29$ (Kawai et al. 2006) also had prompt optical emission observed (Boër et al. 2006, Haislip et al. 2006), with a brightness, single-pulse structure, and fast-fading behavior reminiscent of GRB 990123 and thus, potentially also interpreted as reverse shock emission.

Swift detection of the "naked eye burst" GRB 080319B, which peaked at visual magnitude $V=5.3$ has now delivered the richest dataset, by far, addressing the prompt optical emission and its evolution into a standard fading afterglow (Racusin et al. 2008a, Bloom et al. 2009, Woźniak et al. 2008). This is only partially due to the extreme brightness of the event; the fact that it occurred at an equatorial location, in the night sky above the Western hemisphere, is probably even more important. The fact that it occurred within just one hour and ten degrees of the preceding GRB 080319A means that it is also the only event with strong contraints on optical precursor emission from pointed telescopes (Racusin et al. 2008a). The GRB 080319B dataset is rich enough that its ramifications are still being grappled with. In an overall sense the prompt optical emission correlates well with the $\gamma$-ray lightcurve; in detail, though, the individual pulses

\footnotetext{
${ }^{3}$ The $\sim 60 \%$ of those counterparts not detected are presumably fainter than this.
} 
observed in both bands do not track precisely, probably suggesting the presence of at least two distinct cooling processes at play within the dissipation region (Racusin et al. 2008a).GRB 080319B also allowed astronomers to rule out inverse comptonization as a relevant mechanism (Piran et al. 2009, Zou et al. 2009).

Afterglow emission. The observed properties of the optical afterglow are largely familiar from observations prior to the Swift era (van Paradijs et al. 2000, Mészáros 2002) and will not be reviewed in detail here. An intriguing feature of later-time afterglows, revealed by the rich multiband lightcurves available in the Swift era, has been the occasional presence of chromatic lightcurve breaks, where the X-rays show a clear break (steepening of the flux decay rate) while the optical does not. The break in the X-ray light curve is usually identified with the end of the shallow decay phase. The optical lightcurve follows a single power law decay, usually with a temporal decay index intermediate between those in the X-rays before and after the break. Accommodating such chromatic breaks in a model where the X-ray and optical emissions arise from the same emitting region (Panaitescu et al. 2006) requires not only a temporal evolution of the underlying microphysical conditions within the emitting region but also fine tuning their photon arrival times in such a way that a break in the X-ray will be produced but not at optical wavelengths. Alternatively, the X-ray and optical photons may arise from physically distinct regions, which would naturally account for their seemingly decoupled behavior. Observations of the naked-eye GRB 080319B have sharpened this debate. This is because the optical and X-ray decays in this event exhibit discrepant behavior over two orders of magnitude in time, from $100 \mathrm{~s}$ to a few times $10^{4}$ s post-burst (Racusin et al. 2008a, Kumar and Panaitescu 2008).

Recent results showed that during the initial 500 s of observations $15 \%$ of UVOT lightcurves are seen to rise - with an average peak time of 400 s, $58 \%$ decay from the onset of observations and the remainder are consistent with being flat, but could be rising or decaying (Oates et al. 2009). This leads to a wide range of temporal indices measured before 500s, $-1.17<\alpha<0.21$. Such behavior is also observed by ground based telescopes (Rykoff et al. 2005a, Quimby et al. 2006b, Yost et al. 2006b, Rykoff et al. 2006b) and was seen in pre-Swift observations (Rykoff et al. 2004b). No color evolution was observed during the rising phase of the UVOT lightcurves. One likely scenario is that the rise is caused by the jet plowing into the external medium - the start of the forward shock. If this scenario is correct, then it is possible to determine the Lorentz factor of the jet at the time of the peak, giving $\Gamma \sim 180$ and a lower limit $\Gamma>230$ for those without observed peaks (Oates et al. 2009).

After a few hundred seconds the afterglow decays as a single power-law with a temporal index, measured after 500s, of $-1.20<\alpha<-0.52$. This is consistent with pre-Swift observations and the range is similar to the shallow decay of the XRT canonical model. However, $20 \%$ of UVOT lightcurves are seen with a broken power-law decay after 500s. For these afterglows, the first decay has a range 
between $-0.74<\alpha<-0.46$, which is most consistent with the shallow decay of the X-ray canonical lightcurve and the second decay has a temporal range of $-1.72<\alpha<-1.34$, which is most consitent with the classic afterglow phase.

At late times, $t \gtrsim 1$ day, it was common in the pre-Swift era to observe a steepening of the optical decay to power-law indices $\alpha_{\mathrm{o}}>2$, and to associate this epoch with the "jet break" transition of the underlying, relativistically expanding jet. As jet breaks are intrinsically achromatic, they are expected to manifest in the X-ray light curves from Swift as well as in ground-based optical observations. In fact, over the first three years of Swift operations only a handful of convincing jet-break candidates were identified (Blustin et al. 2006, Stanek et al. 2007, Dai et al. 2007, Willingale et al. 2007, Kocevski and Butler 2008), leading to concerns about the viability of this picture. Some bursts with very long coverage have been convincingly shown to possess no significant breaks, e.g., GRB 060729 (Grupe et al. 2007).

Since then, however, deep optical imaging observations have revealed evidence for jet breaks in several additional Swift afterglows, a significant fraction of those monitored to late times (Dai et al. 2008). Systematic analysis of the Swift XRT data has revealed strong evidence for a jet-break signature in $12 \%$ of the X-ray lightcurves (Racusin et al. 2008b). A consensus has developed that jet breaks for typical Swift bursts may be occurring at late times and faint flux levels that are beyond the limit of the standard ground- and space-based campaigns. Separately, it has been suggested that a distinct additional spectral component could hide the jet-break signature in the X-ray band. For example, in the case of the bright GRB 070125 (Chandra et al.2008), inverse Compton scattering of the synchrotron optical photons has been put forward as an explanation for the missing jet-break feature.

3.2.3 Radio observations. Radio afterglow observations are unique in having led to both indirect and direct demonstrations of relativistic expansion, via scintillation (e.g. Chandra et al. 2008) and VLBI (Taylor et al. 2005) observations. They provide access to the properties of bright afterglows on the smallest angular scales. The limited number of sensitive high-resolution radio facilities, and the sensitivity limits of those facilities, have prevented a proportional exploitation of the greatly-increased burst rate from Swift, primarily because the compensating feature of this increased burst rate has been a greater median redshift and a lower characteristic afterglow flux.

Radio afterglow observations nonetheless continue to play a vital role in accurately estimating blastwave kinetic energies (Oren et al. 2004, Granot et al. 2005a, Kaneko et al. 2007), with radio detections contributing crucially to the demonstration of the extremely large $\left(E \sim 10^{52} \mathrm{erg}\right)$ kinetic energy associated with the high-redshift GRB 050904 (Frail et al. 2006, Gou et al. 2007), and to constraining the relativistic energy associated with the nearby GRB 060218/SN 2006aj (Soderberg et al. 2006b). Radio data can also provide a crucial "third check" on claims of jet-break detections, as with the broadband afterglow models applied to GRB 050820 (Cenko et al. 2006) and GRB 070125 (Chandra et al. 2008). 


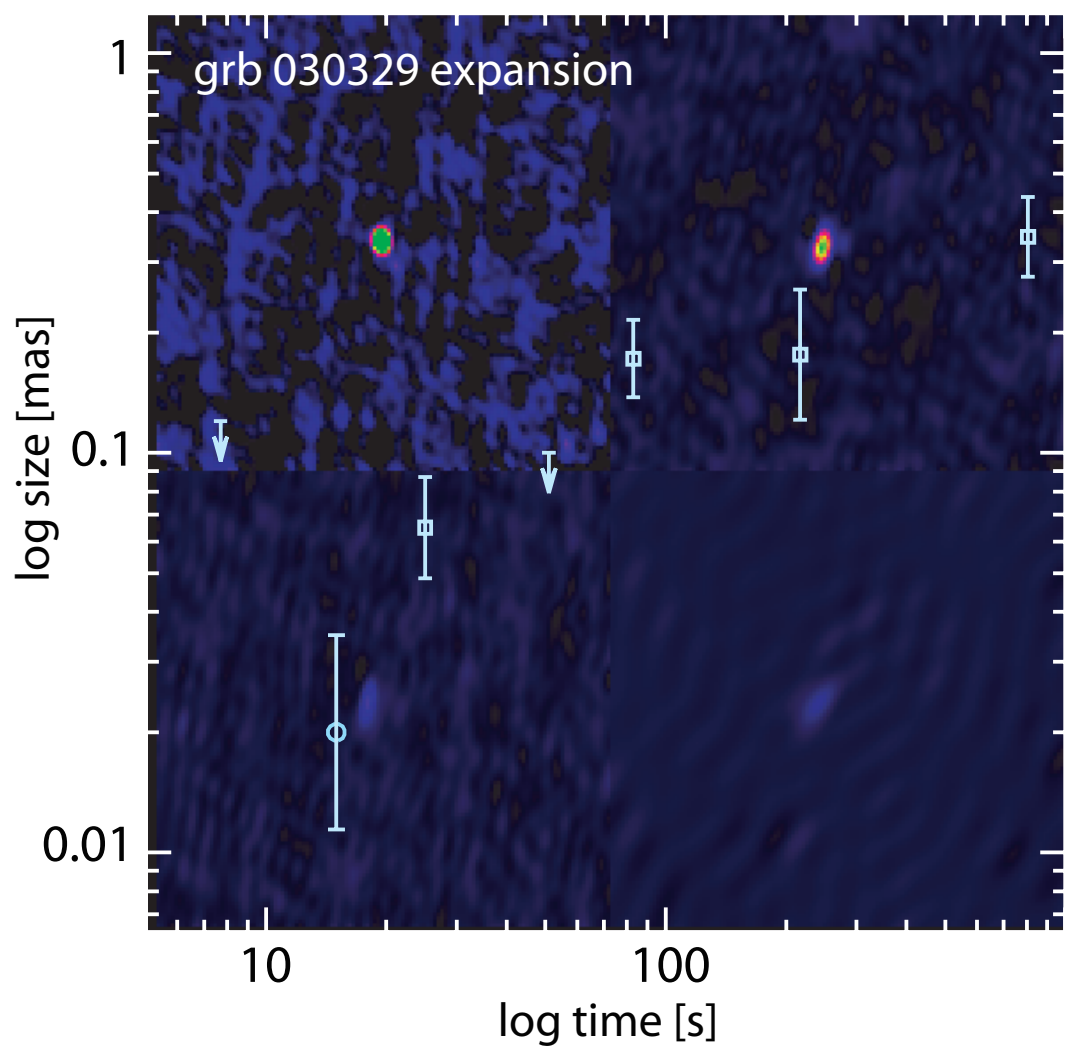

Figure 8 The growth of GRB 030329 with time as measured using VLBI (by Pihlström et al. 2007 and references therein). In the background are the images from (a) April 2003 (15 GHz), (b) June 2003 (8 GHz), (c) November 2003 (8 GHz), and (d) June 2005 (5 $\mathrm{GHz}$ ) with the same intensity scale. The resolution for the four images is not constant in time, but is accounted for in the analysis of the source size.

For relatively nearby GRBs which may be associated with a supernova, radio observations have proven invaluable in providing evidence of the physical expansion of GRB/SNe ejecta through direct imaging. Figure 8 shows the expansion from $\sim 0.02$ mas to $\sim 0.35$ mas of the radio image of GRB 030329 over a time span from $\sim 15$ s after the GRB trigger to $\sim 10^{3}$ s (Pihlström et al. 2007).

\subsection{Interpreting Prompt and Afterglow Emission}

The isotropic-equivalent luminosity of GRB X-ray afterglows scaled to $t \sim 11$ hours after the burst in the source frame, can be used as an approximate estimator for the energy in the afterglow shock for the following reasons (Freedman and Waxman 2001, Piran et al. 2001, Gehrels et al. 2008). First, at 11 hr the X-ray band is typically above the two characteristic synchrotron frequencies, so that the flux has very weak dependence on microphysical parameters and no dependence on the external density, both of which are associated with relatively large uncertainties. Second, at $11 \mathrm{hr}$ the Lorentz factor of the afterglow shock is sufficiently small $(\Gamma \sim 10)$ so that a large fraction of the jet is visible (out to an angle of 


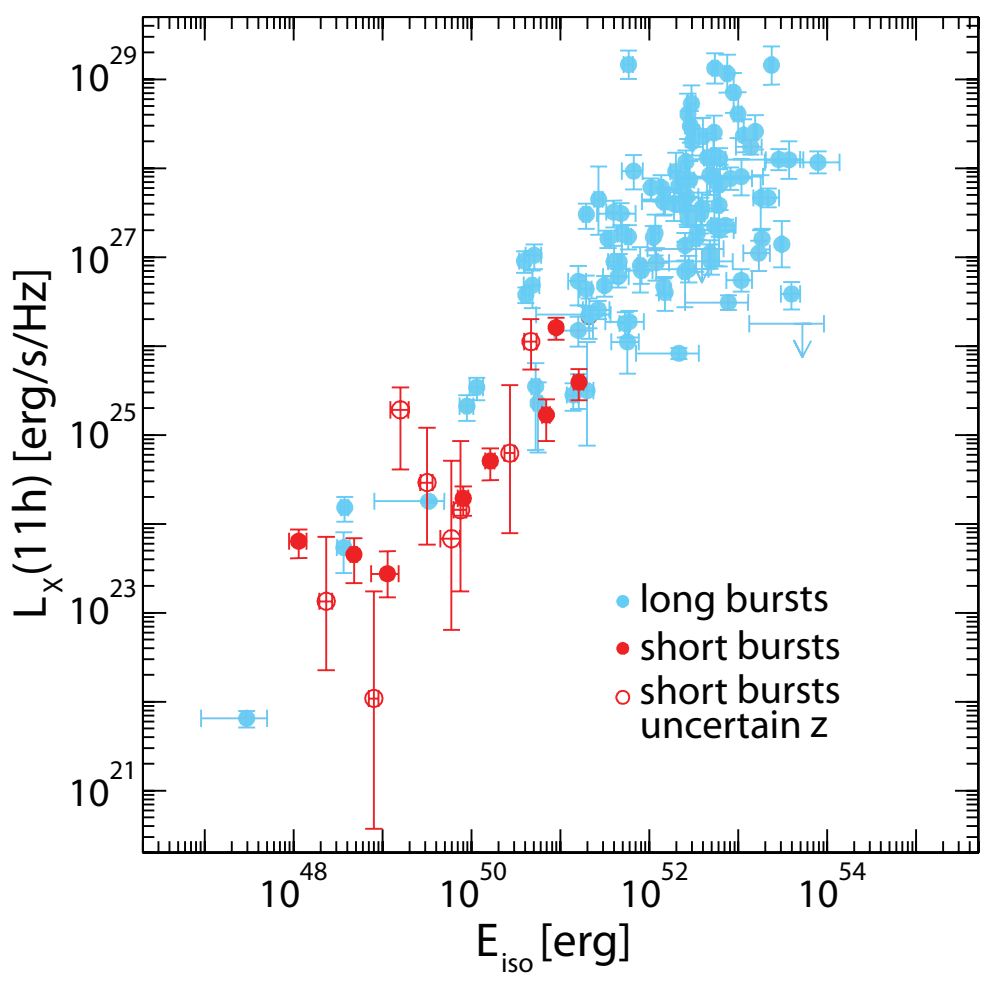

Figure 9 Isotropic-equivalent luminosity of GRB X-ray afterglows scaled to $t=11 \mathrm{hr}$ ( $5 \mathrm{keV}$ source frame) after the burst trigger as a function of their isotropic $\gamma$-ray energy release (adapted from Nysewander et al. 2008).

$\sim \Gamma^{-1} \sim 0.1 \mathrm{rad}$ around the line of sight) and local inhomogeneities on small angular scales are averaged out. Finally, the fact that the ratio of $L_{X}(11 \mathrm{hr})$ and $E_{\text {iso }}$ is fairly constant for most GRBs, suggests that both can serve as a reasonable measure of the isotropic-equivalent energy content of the ejected outflow.

Figure 9 shows $L_{X}(11 \mathrm{hr})$ at $5 \mathrm{keV}$ rest-frame energy as a function of their isotropic $\gamma$-ray energy release for a large sample of GRBs. A linear relation, $L_{X} \propto(11 \mathrm{hr}) \propto E_{\gamma, \text { iso }}$, seems to be broadly consistent with the data, probably suggesting a roughly universal efficiency for converting kinetic energy into $\gamma$-rays in the prompt emission for both long and short GRBs (Lee et al. 2005a, Nousek et al. 2006, Bloom et al. 2007, Kaneko et al. 2007, Nysewander et al. 2008). This "universal" efficiency is also likely to be high (i.e., the remaining kinetic energy is comparable to, or even smaller than, the energy dissipated and radiated in the prompt emission). If this is the case, the well-known efficiency problem for long GRBs also persists for short events.

\subsection{The Radiated Energy Inventory}

With the advent of Swift, the observational inventory for GRBs has become rich enough to allow estimates of their radiated energy content. A compilation of the radiated energy in both the prompt and afterglow phases is presented in Figure 10. 

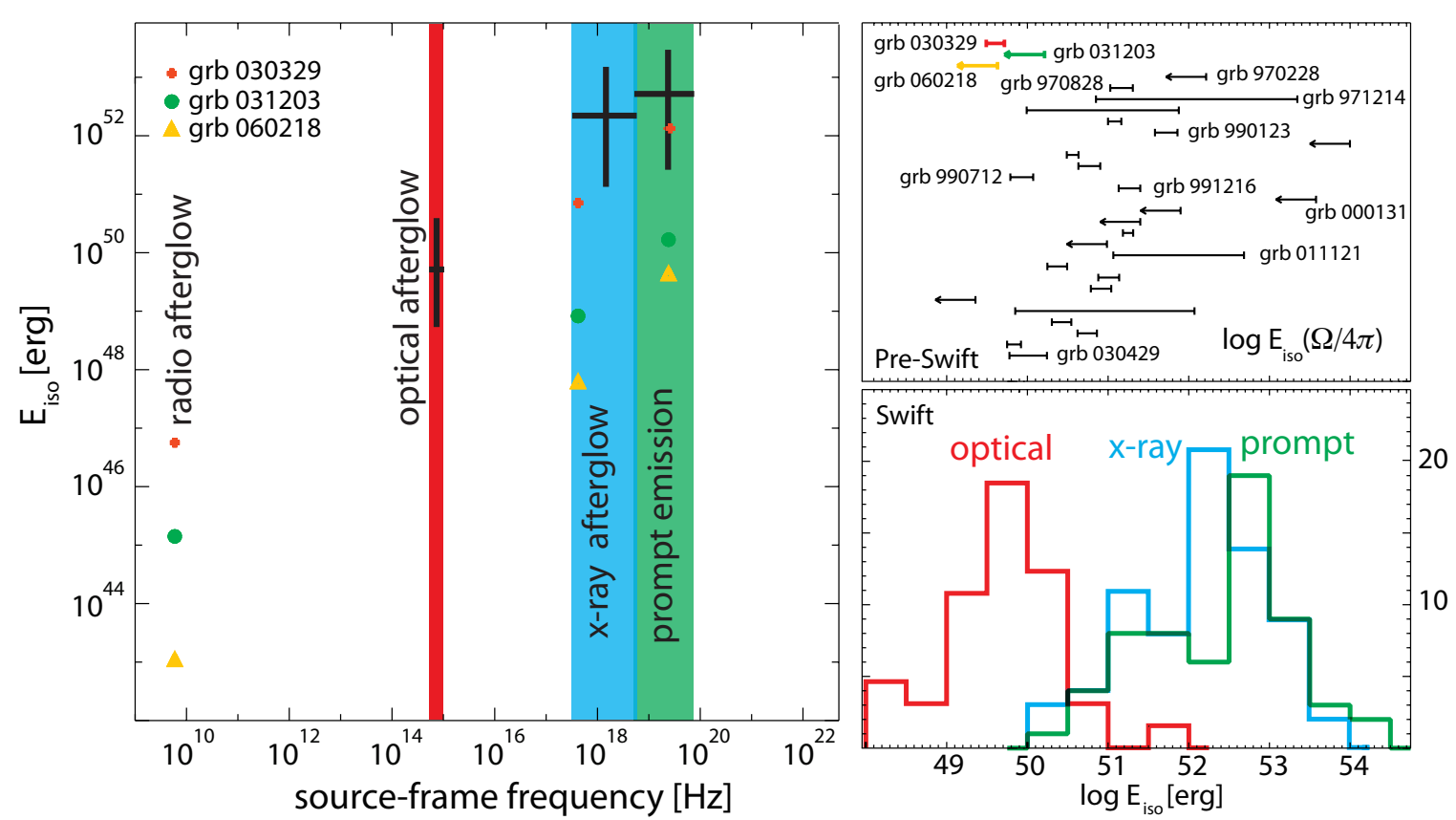

Figure 10 The radiated-energy inventory of Swift GRBs. Left Panel: Summary of the isotropic-equivalent total emitted energy of the prompt and afterglow emission for Swift GRBs in the source frame (adapted from Kaneko et al. 2007). Right Panel Bottom: Distributions of cumulative isotropic-equivalent total emitted energy for Swift GRBs. Right Panel Top: Comparisons of collimation-corrected total emitted $\gamma$-ray energy, $E_{\text {iso }}(\Omega / 4 \pi)$, of pre-Swift GRBs, where $E_{\text {iso }}$ is used as an upper limit for GRBs with no jet angle constraints.

To investigate the energy dissipation behavior in the X-ray and optical afterglow, we fitted a natural cubic spline function to the afterglow histories (shown in Figure 7) for each individual afterglow and estimated the cumulative emitted energy. The start and end times of the integration were the first and the last points of the actual observations. The result is a global portrait of the effects of the physical processes responsible for GRB evolution, operating on scales ranging from AU to parsec lengths. The compilation also offers a way to assess how well we understand the physics of GRBs, by the degree of consistency among related entries.

Figure 10 shows that the isotropic X-ray emission, $E_{\mathrm{X} \text {,iso }}$, for most Swift GRBs spans the range of $\sim 10^{50}$ to $10^{54} \mathrm{erg}$, which is comparable to that emitted during the prompt $\gamma$-ray phase. Not surprisingly, events that have large isotropicequivalent energy in $\gamma$-rays have larger $E_{\mathrm{X} \text {,iso }}$, indicating a reasonably narrow spread in the efficiency of converting the afterglow kinetic energy into radiation. As can be seen also in Figure 10, the isotropic equivalent energy that is radiated at optical wavelengths is $\sim 2$ orders of magnitude smaller than that in X-rays and $\gamma$-rays. This is predominantly due to the fact that $\nu F_{\nu}$ typically peaks closer to the X-rays than to the optical, and it is very flat above its peak while it falls much faster toward lower energies. Finally, since these are isotropic equivalent energies, most of the contribution to the radiated afterglow energy, especially at radio wavelengths, is from significantly later times than for $E_{\mathrm{X} \text {,iso }}$, and the 
collimation of the outflow together with relativistic beaming effects could result in much larger $E_{\mathrm{X} \text {,iso }}$ than $E_{\mathrm{R} \text {,iso }}$.

Of the three nearby SN-GRB events plotted in Figure 10, only GRB 030329 falls within the $\sim 10^{50} \mathrm{erg}$ range, while the other two events fall between $\sim 10^{48}$ and $\sim 10^{49}$ erg (Kaneko et al. 2007). We note that there exists a selection effect based on the observed photon flux: an event is more likely to be detected when it is closer to us than farther, for a given intrinsic luminosity.

One of the liveliest debated issues associated with GRBs concerns the total energy released during the explosion: are GRBs standard candles? The GRB community has vacillated between initial claims that the GRB intrinsic luminosity distribution was very narrow (Horack et al. 1994) to discounting all standardcandle claims, to accepting a standard total GRB energy of $\sim 10^{51}$ ergs (Frail et al. 2001), and to diversifying GRBs into normal and sub-energetic classes (Soderberg et al. 2008, Ramirez-Ruiz et al. 2005a). The important new development is that we now have significant observational support for the existence of a sub-energetic population based on the different amounts of relativistic energy released during the initial explosion. A network of theoretical tests lends credence to this idea (Waxman 2004a, Kaneko et al. 2007, Granot and Ramirez-

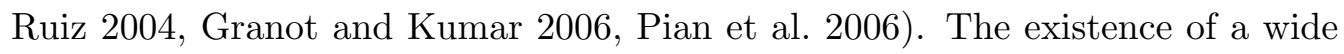
range of intrinsic energies could further challenge the use of GRBs as standard candles.

\section{ENVIRONMENTS AND HOST GALAXIES}

Much of what we know about GRBs has been derived not from observations of the prompt burst radiation itself, but from studies of their afterglows - as they illuminate the circumburst surroundings - and their host galaxies. In the sections that follow we discuss the primary insights that have been derived in this manner from the study of GRB environments.

\subsection{Cosmological Setting}

In Figure 4 we present the redshift distribution of all Swift-detected gamma-ray bursts. Swift and other current missions observe GRBs to cosmological distances quite readily; indeed, the three highest-fluence, known-redshift bursts observed by Swift have been at $z=0.61$ (GRB 050525A), $z=2.82$ (GRB 050603), and $z=1.26$ (GRB 061007) - already spanning 40\% of cosmic history. Historically, the majority of redshifts have been collected via host galaxy spectroscopy; in the Swift era, however, this pattern has been reversed - except for the short bursts - with the great majority of redshifts now being derived from afterglow spectra.

In addition to theoretical arguments that posit different physical origins for short and long bursts (e.g., Katz and Canel 1996), the absence of short burstassociated supernovae to deep limits (Hjorth et al. 2005a, Fox et al. 2005, Bloom and Prochaska 2006, Castro-Tirado et al. 2005), 100 times fainter than SN 1998bw in the best cases, argues for a distinct origin of the short and long bursts. In agreement with this picture, the redshift distributions of the two populations are 
not consistent.

For the long bursts, which are associated with active star formation and, in particular, the deaths of massive stars, it is interesting to explore whether their distribution in redshift is consistent with other measures of cosmic star formation. The greatly-increased number of redshifts available for Swift bursts have motivated several such comparisons, and for the first time, estimates of cosmic star formation at high redshift $z>4$ using the Swift redshift sample (Chary et al. 2007, Yüksel et al. 2008).

The star-formation studies over $1<z<4$ confirm, in a broad sense, that the GRB redshift distribution remains consistent with independent measures of star formation (Jakobsson et al. 2006). However, there are signs of differential evolution of the GRB rate, in the sense that the GRB rate increases more rapidly with increasing redshift than expected based on star-formation measures alone (Le and Dermer 2007, Guetta and Piran 2007, Kistler et al. 2008). This evidence, currently present at roughly $95 \%$-confidence, may strengthen significantly

in coming years. If so, this would provide a sign of bias towards low-mass and low-metallicity host galaxies - and potentially, low-metallicity progenitors - for the long-duration bursts (Section 4.2). The theoretical curves accompanying the GRB redshift distribution (Figure 4) which show the evolution of a comoving volume element in the universe, and the volume convolved with star formation rate, appear to indicate that the observed distribution is wider than expected.

The short-burst redshift distribution, so far drawn exclusively from host-galaxy observations, has also been compared to star-formation metrics. In this case, however, the intent has been to explore "time-delayed" progenitor models that correlate with star formation through a parametrized (log-normal or power-law) delay function (Nakar et al. 2006a, Guetta and Piran 2006, Salvaterra et al. 2008). Consistent with the relatively large fraction of events at $z \lesssim 0.5$ compared to long bursts, and with host galaxy demographics (Zheng and Ramirez-Ruiz 2007, Shin and Berger 2007), these studies have concluded that a long-lived ( $\tau \gtrsim 1$ Gyr) progenitor is required for these models to be consistent with Swift-era redshift measurements and the distribution of short burst fluences from BATSE. This in turn has led to relatively high estimates of the volumetric local short burst rate, at least an order of magnitude greater than the local rate of long bursts (Guetta et al. 2005), and a correspondingly optimistic set of predictions for Enhanced LIGO, VIRGO, and other ground-based gravity-wave detectors.

\subsection{Host Galaxies of Long Bursts}

Surveys of GRB host galaxies in the pre-Swift era (Le Floc'h et al. 2003, Fynbo et al. 2003) necessarily focused on the host galaxies of long-duration bursts. These surveys established a standard picture for the GRB hosts as sub- $L^{*}$ galaxies (median $L \sim 0.1 L^{*}$ ) with exponential-disk light profiles (Conselice et al. 2005, Wainwright et al. 2007) and high specific star-formation rates (SSFR $\sim 1 \mathrm{Gyr}^{-1}$ ) (Christensen et al. 2004). A selection of GRB host galaxies, as imaged by HST, are shown in Figure 11 .

This picture has not substantially changed in the Swift era. To the contrary, 

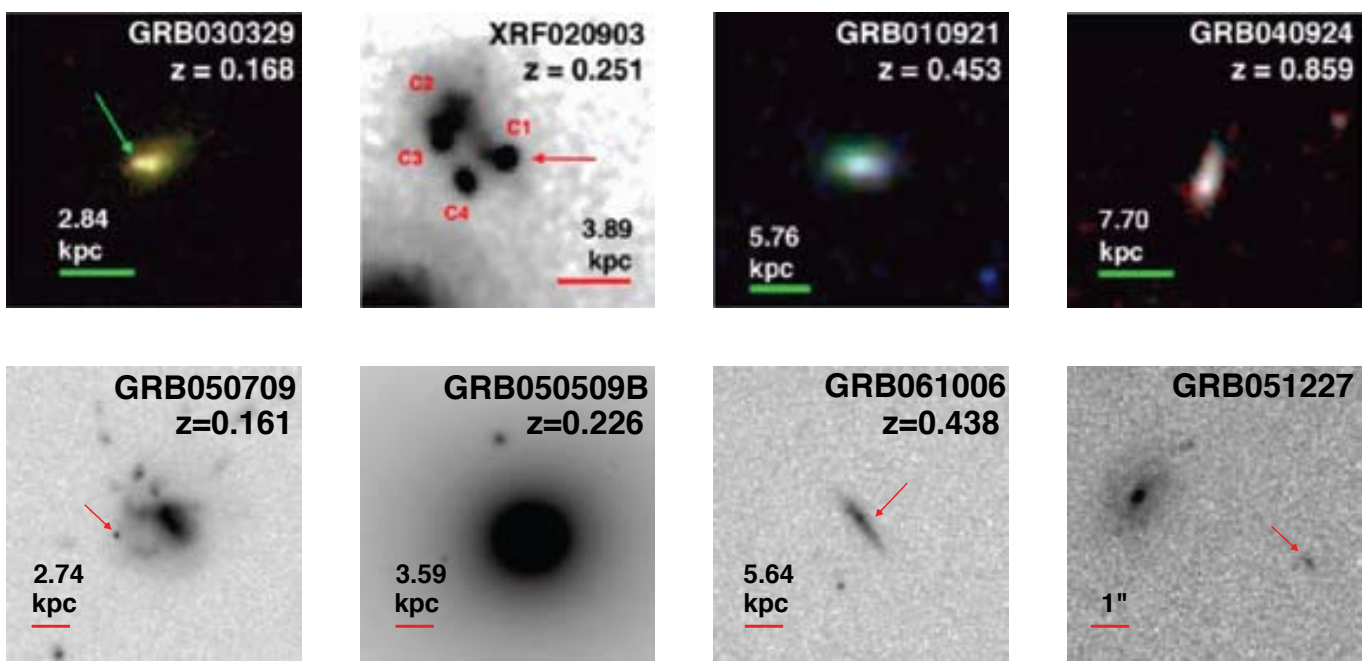

Figure 11 A selection of the host galaxies of long-duration (top row) and short-duration (bottom row) gamma-ray bursts, as imaged by the Hubble Space Telescope. An attempt has been made to choose pairs of long and short burst host galaxies with comparable redshifts; lower-redshift hosts are emphasized as these reveal their structure more readily in short exposures. Images are oriented with North up and East to the left, and the physical length scale for a one-arcsecond angular distance is indicated in each panel (except for GRB 051227); arrows point to the location of the burst where this is known to pixel precision. Individual burst notes: GRB 030329 was the first classical long GRB to be associated with a well-observed spectroscopic supernova (Stanek et al. 2003, Hjorth et al. 2003); XRF 020903 was the first X-ray Flash event to yield a redshift measurement Soderberg et al. (2004); GRB 050709 was the first short burst with optical afterglow indicated by the arrow - detected Fox et al. (2005), Hjorth et al. (2005b); GRB 050509B was the first short burst with detected afterglow (Gehrels et al. 2005, Bloom et al. 2006) GRB 051227 has a faint candidate host, of unknown redshift probably greater than 1 , visible at the optical afterglow location; the spiral galaxy to the east has redshift $z=0.714$ Foley et al. (2005). Long-burst host images from Wainwright et al. (2007); short-burst host images from Fox et al. (2005) and this work.

the result of various ground- and space-based efforts to characterize GRB host galaxies (Le Floc'h et al. 2006, Chary et al. 2007, Fynbo et al. 2008, Savaglio et al. 2009) have confirmed this basic outline and expanded its domain of applicability to high redshift, combining the power of the Swift burst catalog with Spitzer observations. At higher redshifts, $z \gtrsim 3$, it seems particularly interesting to use the GRB host galaxies to explore the evolution of mass-metallicity relationships that are typically compiled using field galaxies at low redshift and high-mass galaxy samples at high-redshift (Figure 12). Since GRB host redshifts are typically secured via afterglow spectroscopy, the hosts themselves are uniquely free of mass and luminosity selection effects. In this context, even upper limits provide useful constraints on mass-metallicity correlations and their evolution with redshift (Chary et al. 2007, Savaglio et al. 2009, Berger 2009). Such studies have also served to place GRB host galaxies in the context of other 


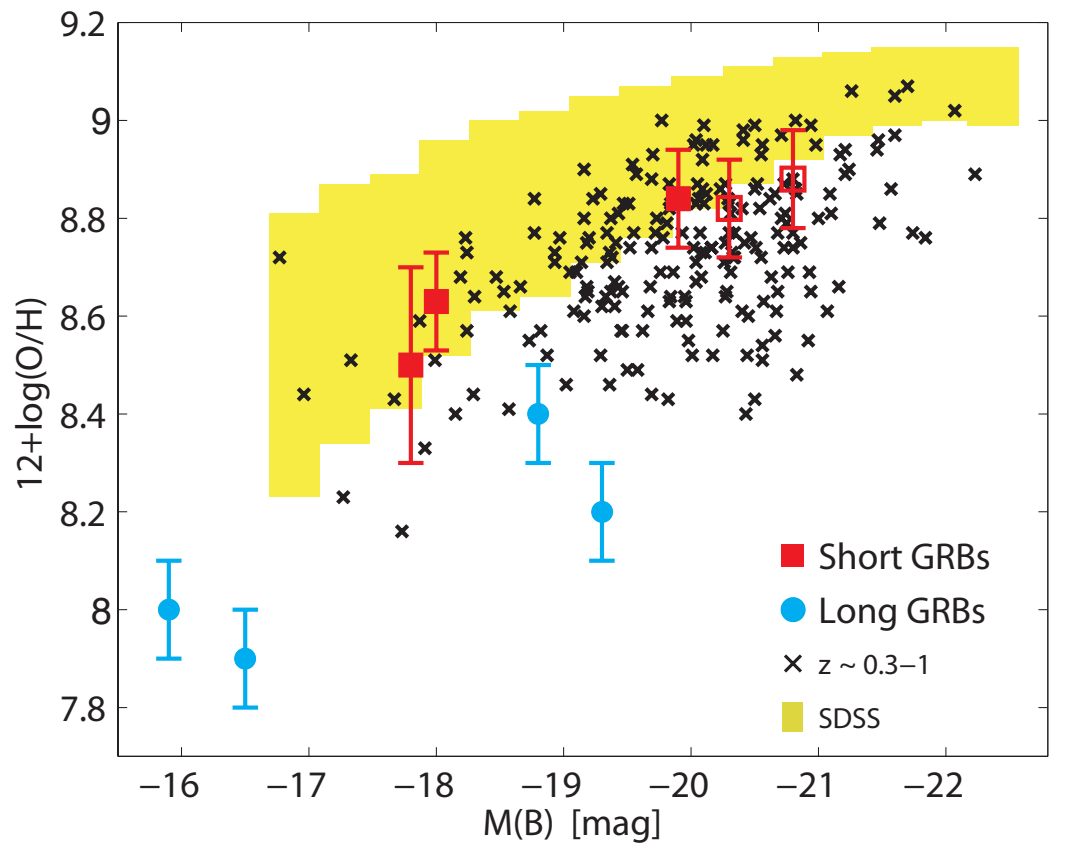

Figure 12 Metallicity as a function of $B$-band absolute magnitude for the host galaxies of short (red) and long (blue) GRBs. The yellow bars mark the 14-86 percentile range for galaxies at $z \sim 0.1$ from the Sloan Digital Sky Survey (Tremonti et al. 2004), while crosses designate field galaxies at $z \sim 0.3-1$ (Kobulnicky and Kewley 2004). Both field samples exhibit a clear luminosity-metallicity relation. The long GRB hosts tend to exhibit lower than expected metallicities (Stanek et al. 2006), while the hosts of short GRBs have higher metallicities by about 0.6 dex and are moreover in excellent agreement with the luminosity-metallicity relation. From Berger (2009).

high-redshift galaxy populations incuding the Lyman-break and Lyman-alpha emitting galaxies (Fynbo et al. 2008).

Finally, it is somewhat reassuring that numerical simulations of star-forming galaxy populations can generate galaxy subpopulations that reproduce the basic properties of the GRB host galaxies in several important respects (Courty et al. 2004, Courty et al. 2007). In particular, selecting for high specific star-formation rate generates mock galaxy catalogs with similar masses, luminosities, and colors to GRB host galaxies.

4.2.1 Metallicity matters. While GRB host galaxies are often studied for the insights they provide about larger astrophysical questions, including the history of star formation through cosmic time, they also have the potential to shed light on the nature of the GRB progenitors. The association of long-duration bursts with star formation, for example, was proposed after observation of just two host galaxies (Paczyński 1998), and demonstrated firmly from the properties of the first twenty (Bloom et al. 2002 ).

Recent years have seen a surge of interest in the question of whether GRB host galaxies, and hence, presumably, GRB progenitors, are metal-poor by comparison to the larger population of star-forming galaxies. This question has been explored from a variety of perspectives, and the result of these studies, still under active 
debate, may eventually help refine our picture of the massive stellar death behind each GRB.

The metallicities of GRB host galaxies, and indeed, detailed abundance profiles, can be measured directly from high-resolution spectroscopy of bright afterglows. This area has seen dramatic progress in the Swift era, with prompt arcsecond positions and visual magnitude measurements from Swift UVOT and groundbased robotic telescopes feeding rapid-response spectroscopy from large-aperture facilities. One chief result of these efforts have been the collection of detailed abundance characterizations for multiple bursts (Vreeswijk et al. 2004, Chen et al. 2005, Fynbo et al. 2006a, Bloom et al. 2007, Prochaska et al. 2007, Chen et al. 2007, Price et al. 2007, Chen et al. 2008). In addition, the metallicities of GRB host galaxies have been measured via standard emission-line diagnostics (Stanek et al. 2006, Thöne et al. 2008, Savaglio et al. 2009), where possible, and in some cases tentative conclusions have been drawn on the basis of cruder relations such as mass-metallicity metrics (Berger et al. 2007a) and host galaxy luminosities and morphologies (Fruchter et al. 2006).

The conclusions of these studies have yet to be reconciled into a single coherent picture of the nature of the GRB host galaxies and their relationship to other low- and high-redshift galaxy populations. However, the wealth of data do serve to define some associated issues with reasonable clarity. First, the metallicities of GRB host galaxies at $z \lesssim 1$ are significantly $\left(Z \sim 0.1 Z_{\odot}\right)$ sub-solar (Savaglio et al. 2009), consistent with the sub-solar metallicities measured for GRB host galaxies via absorption spectroscopy at $z \gtrsim 2$ (e.g., Chen et al. 2008). These sub-solar metallicities are neither surprising nor unusual for galaxy populations at high redshift (Fynbo et al. 2008, Savaglio et al. 2009); moreover, several candidate higher-metallicity hosts have been identified, although not yet confirmed (Berger et al. 2007a, Fruchter et al. 2006). At the same time, GRB host galaxies seem to be readily distinguished, in luminosity and morphology, from the host galaxies of core-collapse supernovae at similar redshifts (Fruchter et al. 2006), and the host galaxies of the lowest-redshift $z \lesssim 0.2$ bursts have uniformly low metallicities that strongly distinguish them from the bulk of the low-redshift galaxy population (Stanek et al. 2006, Thöne et al. 2008), and indeed, from the host galaxies of nearby type Ibc supernovae (Modjaz et al. 2008).

These somewhat divergent findings might be reconciled in a picture where the GRB progenitors prefer (or require) a low-metallicity environment, since the increasing prevalence of such environments at $z \gtrsim 1$ would allow GRB host galaxies to present an increasingly fair sample of the population of star-forming galaxies at these higher redshifts. This argument would also dovetail with observations that the GRB rate seems to increase with redshift faster than the cosmic star formation rate, as mentioned above (Le and Dermer 2007, Guetta and Piran 2007, Kistler et al. 2008). However, the claim that GRB host galaxies represent a fair sample of star-forming galaxies, even at $z \gtrsim 1$, remains in dispute (e.g., Figure 12, Berger 2009).

A possibly significant implication of a metallicity-dependent GRB rate would be an offset between the true star-formation rate and that traced by GRBs. 
If GRBs in low-metallicity environments and low-mass galaxies are more luminous, then they are likely over-represented in GRB samples. Low-mass galaxies and galaxy outskirts have lower metallicity on average and thus may yield more (and/or more luminous) GRBs compared to high-mass galaxies (Ramirez-Ruiz et al. 2002b). As galaxy mass builds up through mergers, it is also possible that the highest- $z$ GRBs could be systematically more luminous due to their lowermass host galaxies, an intriguing hypothesis given the extreme luminosities of some of the highest-redshift bursts of the Swift era, including GRB 050904.

Finally, it is worth noting that high-resolution afterglow spectra show absorption features imprinted on the afterglow by gas at multiple, widely divergent physical scales, possibly extending from $d \sim 10 \mathrm{pc}$ to Gpc distances (Section 4.2.2). With the GRB metallicity question ultimately referring to the nature of the progenitor itself, searches for definitive signatures of the progenitor's stellar wind material (Möller et al. 2002, Schaefer et al. 2003, Mirabal et al. 2003, Bloom et al. 2007, Chen et al. 2007, Fox et al. 2008, Prochaska et al. 2008) should continue to be pursued and refined. At the same time, in discussing the host galaxies as a population, more common galaxy-integrated, emission-line diagnostics may better serve to place GRB hosts in the proper cosmological context.

4.2.2 Subgalactic environments. The same fast-response spectra that have enabled characterization of elemental abundances in GRB host galaxies have led to a series of discoveries regarding absorbing gas structures on sub-galactic scales within the GRB host: a rich array of high-ionization fine-structure Fe transitions in GRB 051111 (Berger et al. 2005a, Penprase et al. 2006, Prochaska et al. 2006); discovery of time-variability of such fine structure features in three bursts, demonstrating excitation by UV photons from the burst flash and young afterglow (Della Valle et al. 2006b, Vreeswijk et al. 2007, D'Elia et al. 2008); discovery of high-ionization N V features, providing evidence for absorption by gas within $d \lesssim 10$ pc of the burst (Fox et al. 2008, Prochaska et al. 2008); and most recently, the first detection of molecular gas along the line of sight to a GRB afterglow (Figure 13, Prochaska et al. 2009).

The range of these discoveries reveals a surprising complication in the interpretation of afterglow spectra. Depending on the burst, it may be necessary to account for absorbing structures on every scale from the immediate parsec-size circumburst environment, to surrounding or intervening molecular clouds, to host galaxy absorbers on kiloparsec scales, and ultimately, to low-ionization gas associated with cosmological structures at gigaparsec distances. Similar complications are a feature of quasar absorption line studies; there, however, the situation is simplified by the persistent nature and overwhelming ionizing power of the quasar itself.

Indeed, the situation may be even more complicated than this. The discovery that the frequency $(d N / d z)$ of strong Mg II absorbers along GRB lines of sight seems to be roughly four times the frequency along quasar lines of sight (Prochter et al. 2006) suggests that some or most such absorbers seen toward GRBs despite their large separation in redshift from the host galaxy - may be intrinsic 

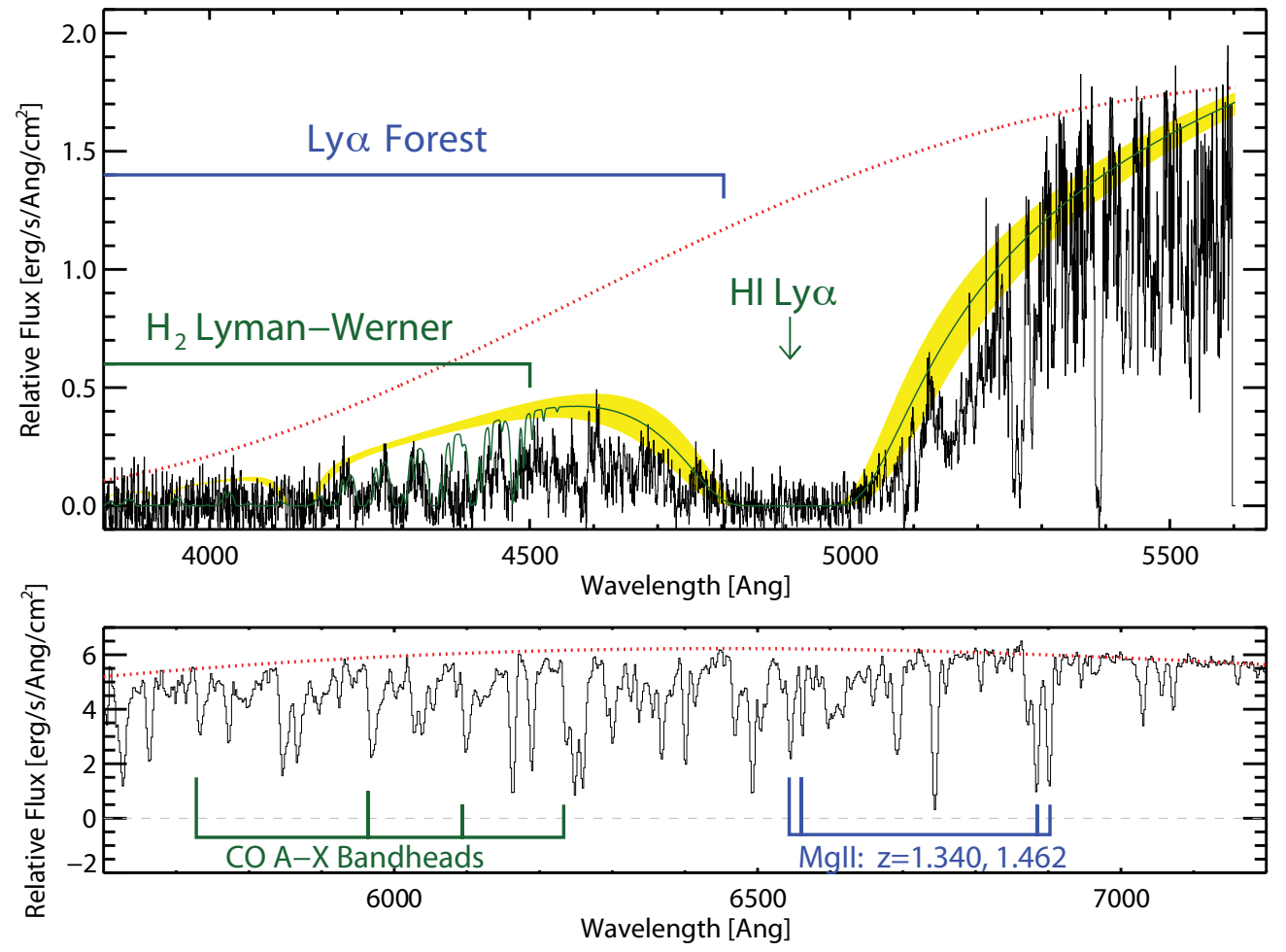

Figure 13 Keck/LRIS spectrum of the afterglow for GRB 080607 (Prochaska et al.|2009). The red dashed lines indicate a model of the intrinsic afterglow spectrum reddened heavily by dust in the host galaxy (rest-frame $A_{V} \approx 3.2$ ). At $\lambda \approx 4900 \AA$ one identifies a damped Ly $\alpha$ profile associated with H I gas near the GRB. The shaded region overplotted on the data corresponds to an H I column density $N_{H}=10^{22.7 \pm 0.15} \mathrm{~cm}-2$. The model (green solid line) includes absorption from $\mathrm{H}_{2}$ Lyman-Werner transitions. The line opacity at $\lambda>5500 \AA$ is dominated by metal-line transitions from gas in the host galaxy and includes bandheads of the CO molecule. Suprisingly, this is the only sightline to date to show strong molecular absorption (Tumlinson et al. 2007). It also exhibits a roughly solar metallicity. The figure also shows the spectral region or features corresponding to intergalactic Ly $\alpha$ and MgII absorption. The redshift for GRB 080607 is $z=3.036$.

to the GRB environment (Porciani et al. 2007).

\subsection{Host Galaxies of Short Bursts}

At the time of the Swift launch, the greatest mystery in GRB astronomy was the origin of short GRBs. A major step forward was made in summer 2005 with the localization and afterglow detection of three short bursts, GRB 050509B, 050709 and 070724 . These events were found to be localized in regions of low star formation, either in low star forming elliptical galaxies as for GRB 050509B Gehrels et al. 2005, Bloom et al. 2006) and GRB 050724 Barthelmy et al. 2005c, Berger et al. 2005b) or in a region of a galaxy with low star formation (Villasenor et al. 2005, Fox et al. 2005, Hjorth et al. 2005b). This was in stark contrast to long bursts which are associated with star forming regions, and implied a non 
massive core-collapse origin.

More than three years after these first short burst localizations, the catalog of confidently-identified short burst host galaxies is growing to the point where systematic studies can be carried out (Berger 2009) - although the effects of uncertain burst attribution (i.e., long or short?), uncertain host identification (especially for bursts with only Swift BAT or XRT localizations), and unknown redshifts for faint candidate hosts conspire to keep any conclusions largely qualitative at this time.

Indeed, without direct afterglow spectroscopy, association of short bursts with candidate host galaxies and host galaxy clusters must be approached probabilistically. In cases where a well-localized (preferably sub-arcsec) afterglow falls on a luminous region of the candidate host, or within a high-mass or high-redshift cluster, the association can probably be considered secure; however, in other cases an a posteriori estimate of the probability of association must be made (Bloom and Prochaska 2006, Fox and Roming 2007). Such estimates are inevitably strongly dependent on input assumptions. For example: What lifetime and kick velocity distributions might be appropriate for progenitor binary systems? What about other possible progenitor classes? Any assumptions must be carefully considered before and after they are applied.

With these caveats, a picture of the short burst host galaxy population as a whole has developed (Figure 11). It consists of three classes of host, two of which became apparent soon after the short burst afterglow revolution of 2005: the low-redshift $(z \lesssim 0.5)$, high-mass $\left(L \sim L^{*}\right)$, early-type host galaxies and galaxy clusters, on the one hand (Gehrels et al. 2005, Fox et al. 2005, Bloom et al. 2006, Berger et al. 2005b, Gal-Yam et al. 2008), typified by the hosts of GRB 050509B, GRB 050724, and GRB 050813; and the low-redshift, sub- $L^{*}$, late-type galaxies on the other, typified by the hosts of GRB 050709 (Fox et al. 2005 Covino et al. 2006), GRB 051221 Soderberg et al. (2006a), and GRB 061006 D'Avanzo et al. (2009).

The third class of short burst host galaxies consists of faint, star-forming galaxies at $z \gtrsim 1$ (Berger et al. 2007b, Cenko et al. 2008, Berger 2009), reminiscent of the host galaxies of long bursts. The existence of such higher-redshift, starforming short burst host galaxies was predicted (Belczynski et al. 2006) based on binary population synthesis models assuming the compact object merger model for the short bursts. These simulations yield a bimodal distribution of lifetimes for merging systems, with a spike of mergers at short timescales, $\tau \lesssim 100 \mathrm{Myr}$, followed by a dominant merger population with a $\tau^{-1}$ lifetime distribution.

The association of short GRBs with both star-forming and early-type galaxies has led to analogies with type Ia supernovae, whose host demographics have similarly provided evidence for a wide distribution of delay times between formation and explosion. At the same time, both core-collapse supernovae and long-duration GRBs are observed (almost) exclusively in late-type star-forming galaxies. As with supernovae and long-duration bursts, a detailed census of short burst redshifts, host galaxy types, and burst locations within those hosts will undoubtedly help to constrain progenitor models. 


\subsection{Neither Long Nor Short}

Some interesting gamma-ray burst host galaxies are not obviously associated with either the long or short burst classes. In fact, the "peculiar" cases of GRB 060505 and GRB 060614 probably provide the first examples where studies of the host galaxy properties have been applied to argue for a (long- or short-) nature of the bursts themselves (Fynbo et al. 2006b, Gal-Yam et al. 2006, Ofek et al. 2007, Thöne et al. 2008).

GRB 060614 was a low-redshift, long-duration burst with no detection of a coincident supernova to deep limits. It was a bright burst (fluence in $15-150$ $\mathrm{keV}$ band of $2.2 \times 10^{-5} \mathrm{erg} \mathrm{cm}^{-2}$ ) and well-studied in the X-ray and optical. With a T90 duration of $102 \mathrm{~s}$, it seems to fall squarely in the long burst category. A host galaxy was found (Gal-Yam et al. 2006, Fynbo et al. 2006b, Della Valle et al. 2006a) at $z=0.125$ and deep searches were made for a coincident supernova. All other well-observed nearby GRBs have had supernovae detected, but this one did not to limits $>100$ times fainter than previous detections.

GRB 060614 shares some characteristics with short bursts (Gehrels et al. 2006). The BAT light curve shows a first short, hard-spectrum episode of emission (lasting $5 \mathrm{~s}$ ) followed by an extended and somewhat softer episode (lasting 100 s). The total energy content of the second episode is 5 times that of the first. Its light curve shape is similar in many respects to that of short bursts with extended emission. There are, however, differences in that the short episode of this event is longer than the previously detected examples and the soft episode is relatively brighter. Another similarity with short bursts comes from a lag analysis of GRB 060614. The lag between temporal structures in the $50-100 \mathrm{keV}$ band and those in the $15-25 \mathrm{keV}$ bands for the first $5 \mathrm{~s}$ is $3 \pm 6 \mathrm{~ms}$ which falls in the same region of the lag-luminosity plot as short bursts (Figure 5). It is difficult to determine unambiguously which category of burst GRB 060614 falls into. It is a long event by the traditional definition, but it lacks an associated SN as had been observed in all other nearby long GRBs. It shares some similarities with Swift short bursts, but has important differences such the brightness of the extended soft episode. If it is due to a collapsar, it is the first indication that some massive star collapses either fail as supernovae (Woosley 1993) or highly underproduce ${ }^{56} \mathrm{Ni}$ (Lopez-Camara et al. 2008); if it is due to a merger, then the bright long-lived soft episode is hard to explain within the framework of compact binary mergers (Di Matteo et al. 2002, Lee et al. 2004, Setiawan et al. 2004). Thus, this peculiar burst has challenged the usual classifications of GRBs.

\section{BASIC PHYSICAL CONSIDERATIONS}

In this section, we endeavor to outline some of the physical processes that are believed to be most relevant to interpreting GRBs. Though the field is far from maturity, sufficient progress has been made in identifying the essential physical ingredients. A basic scheme can provide a conceptual framework for describing the observations even when the framework is inaccurate! The following should be interpreted in this spirit. 

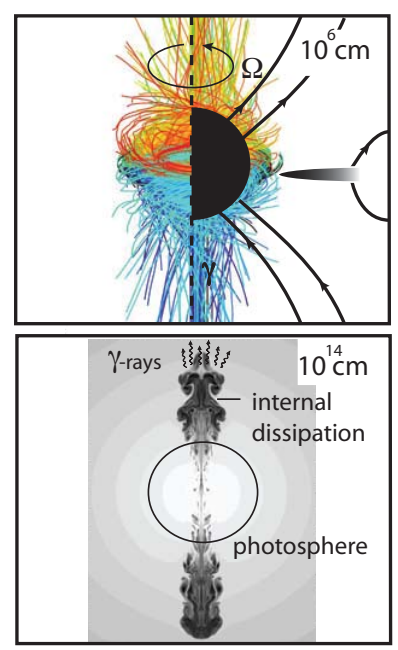
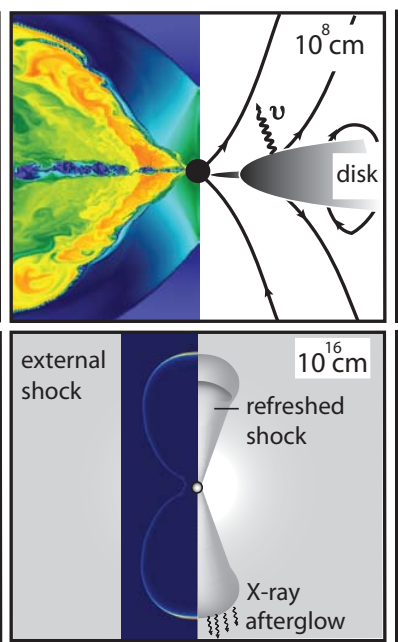
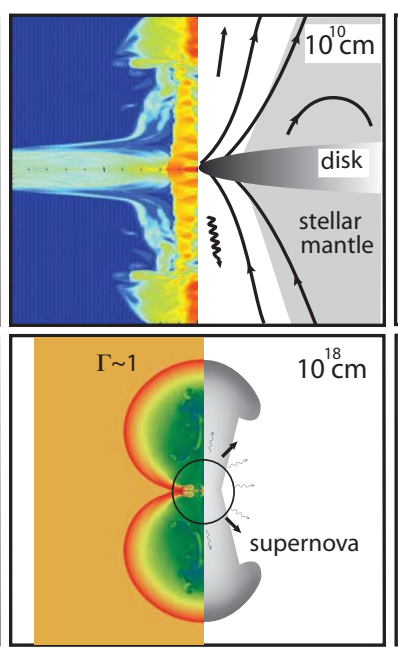
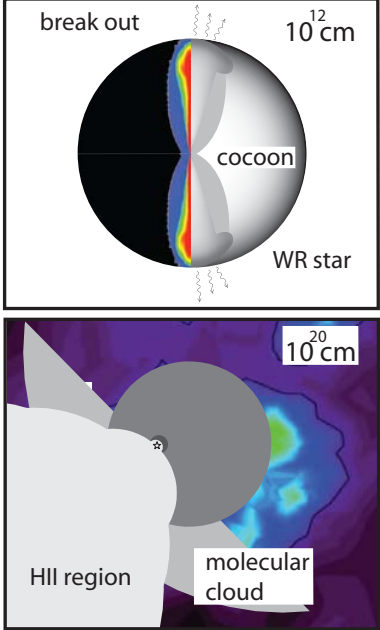

Figure 14 Diagram exhibiting GRB activity over successive decades in radius ranging from $10^{6} \mathrm{~cm}$ to $1 \mathrm{pc}$. The first task in attempting to construct a general scheme of GRBs is to decide which parameters exert a controlling influence upon their properties. $\left(10^{6} \mathrm{~cm}\right)$ The GRB nucleus (BH or NS) and its magnetosphere. $\left(10^{8} \mathrm{~cm}\right)$ The accretion flow is likely to be embedded in a very active corona. We expect coronal arches, as well as large magnetic structures, to be quite common and to be regenerated on an orbital timescale. Relativistic outflow from the black hole is proposed to be focused into two jets. $\left(10^{10} \mathrm{~cm}\right)$ Even if the outflow is not narrowly collimated, some beaming is expected because energy would be channelled preferentially along the rotation axis. The majority of stellar progenitors, with the exception of some very compact stars, will not collapse entirely during the typical duration of a GRB. A stellar envelope will thus remain to impede the advance of the jet. $\left(10^{12} \mathrm{~cm}\right)$ This is the typical size of an evolved massive star progenitor. A thermal break-out signal should precede the canonical, softer $\gamma$-rays observed in GRBs. $\left(10^{14} \mathrm{~cm}\right)$ Velocity differences across the jet profile provide a source of free energy. The most favourable region for shocks producing highly variable $\gamma$-ray light curves is above the baryonic or pair-dominated photosphere. $\left(10^{16} \mathrm{~cm}\right)$ The external shock becomes important when the inertia of the swept-up external matter starts to produce an appreciable slowing down of the ejecta. $\left(10^{18} \mathrm{~cm}\right)$ Finally, we come to the end of the relativistic phase. This happens when the mass $E / c^{2}$ has been swept-up.

GRB activity manifests itself over a dynamical range of $\sim 13$ decades in radius. In Figure 14, we show a schematic montage of successive decades, exhibiting phenomena which are believed to take place on each of these length scales. The phenomena are not directly observed and the associated frames represent educated guesses of their geometrical arrangements. An anatomical summary of the underlying physical processes working outward from the smallest to the largest scales follows.

\subsection{The Central Engine}

In principle, flow onto a compact object can liberate gravitational potential energy at a rate approaching a few tenths of $\dot{M} c^{2}$, where $\dot{M}$ is the mass inflow 
rate. Even for such high efficiencies the mass requirements are rather large, with the more powerful GRB sources $\sim 10^{53} \mathrm{erg} \mathrm{s}^{-1}$ having to process upwards of $10^{-2} M_{\odot} \mathrm{s}^{-1}$ through a region which is not much larger than the size of a NS or a stellar mass $\mathrm{BH}$. Radiation of the $\mathrm{BH}$ rest mass on a timescale $r_{\mathrm{g}} / c$, where $r_{\mathrm{g}}=G M / c^{2}=1.5 \times 10^{5}\left(M / M_{\odot}\right) \mathrm{cm}$ is the characteristic size of the collapsed object, would yield luminosities $c^{5} / G=4 \times 10^{59} \mathrm{erg} \mathrm{s}^{-1}$.

When mass accretes onto a $\mathrm{BH}$ or NS under these conditions, the densities and temperatures are so large that photons are completely trapped and neutrinos, being copiously emitted, are the main source of cooling. The associated interaction cross section is then many orders of magnitude smaller, and, as a result, the allowed accretion rates and luminosities are correspondingly much higher. For example, using the cross section for neutrino pair production ${ }^{4}$, the Eddington limit can be rewritten as $L_{\mathrm{Edd}, \nu}=8 \times 10^{53}\left(E_{\nu} / 50 \mathrm{MeV}\right)^{-2}\left(M / M_{\odot}\right) \mathrm{erg} \mathrm{s}^{-1}$, with an associated accretion rate is $\dot{M}_{\mathrm{Edd}, \nu} \times(\text { efficiency })^{-1}$, where $\dot{M}_{\mathrm{Edd}, \nu}=L_{\mathrm{Edd}, \nu} / c$ (Ramirez-Ruiz 2006b, Lee and Ramirez-Ruiz 2007).

The blackbody temperature if a luminosity $L_{\mathrm{Edd}, \nu}$ emerges from a sphere of radius $5^{5} r_{\mathrm{g}}$ is

$$
T_{\mathrm{Edd}, \nu}=\left(\frac{L_{\mathrm{Edd}, \nu}}{4 \pi r_{\mathrm{g}}^{2} \sigma_{\mathrm{SB}}}\right)^{1 / 4} \sim 45\left(M / M_{\odot}\right)^{-1 / 4}\left(\frac{E_{\nu}}{50 \mathrm{MeV}}\right)^{-1 / 2} \mathrm{MeV} .
$$

The radiation temperature is expected to be $\leq T_{\mathrm{th}}=G M m_{\mathrm{p}} /\left(3 k r_{\mathrm{g}}\right) \sim 200 \mathrm{MeV}$, the temperature the accreted material would reach if its gravitational potential energy were turned entirely into thermal energy.

Related to this, there is a fiducial density in the vicinity of the $\mathrm{BH}$

$$
\rho_{\mathrm{Edd}, \nu}=\frac{\dot{M}_{\mathrm{Edd}, \nu}}{4 \pi r_{\mathrm{g}}^{2} c} \sim 10^{11}\left(M / M_{\odot}\right)^{-1}\left(\frac{E_{\nu}}{50 \mathrm{MeV}}\right)^{-2} \mathrm{~g} \mathrm{~cm}^{-3} .
$$

It should be noted that the typical Thomson optical depth under these conditions is $\tau_{\mathrm{T}} \sim n_{\mathrm{Edd}, \nu}^{1 / 3} r_{\mathrm{g}} \sim 10^{16}$ and so, as expected, photons are incapable of escaping and constitute part of the fluid. A characteristic magnetic field strength is that for which $B^{2} / 8 \pi=n_{\mathrm{Edd}, \nu} m_{p} c^{2}$ :

$$
B_{\mathrm{Edd}, \nu}=\left(\frac{L_{\mathrm{Edd}, \nu}}{R_{\mathrm{g}}^{2} c}\right)^{1 / 2} \sim 3 \times 10^{16}\left(M / M_{\odot}\right)^{11 / 2}\left(\frac{E_{\nu}}{50 \mathrm{MeV}}\right)^{-1} \mathrm{G} .
$$

If a field $B_{\mathrm{Edd}, \nu}$ were applied to a $\mathrm{BH}$ with $J \approx J_{\max }$, the electromagnetic power extraction would be $\sim L_{\mathrm{Edd}, \nu}(\mathrm{Usov} 1992)$.

\subsection{Accretion Flows}

As we discussed above, a BH or NS embedded in infalling matter offers a more efficient power source than any other conceivable progenitor. Although this efficiency is over a hundred times larger than that traditionally associated with

\footnotetext{
${ }^{4}$ Although we have considered here the specific case of neutrino pair creation, the estimates vary little when one considers, for example, coherent scattering of neutrinos by nuclei and/or free nucleons (except for the energy scaling).

${ }^{5}$ Similar overall fiducial numbers also hold for neutron stars, except that the simple mass scalings obtained here are lost.
} 
thermonuclear reactions, the required rate of mass supply for a typical GRB is of course colossal. Such high mass fueling rates are never reached for BHs in XRBs or AGN, where the luminosity remains well below the photon Eddington limit. They can, however, be achieved in the process of forming NS and stellarmass BHs during the collapse of massive stellar cores (Houck and Chevalier 1991. Woosley 1993, MacFadyen and Woosley 1999, Narayan et al. 2001) and in binary mergers involving compact objects (Ruffert et al. 1997, Kluzniak and Lee 1998, Rosswog and Liebendörfer 2003, Lee et al. 2005b, Setiawan et al. 2006, Metzger et al. 2008, Rosswog et al. 2004). Consequently, most recent theoretical work has been directed towards describing the possible formation channels for these systems (Fryer et al. 1999, Belczynski et al. 2002, Izzard et al. 2004, Podsiadlowski et al. 2004). This involves evaluating those which are likely to produce a viable central engine, and understanding the flow patterns near relativistic objects accreting matter in the hypercritical regime (Di Matteo et al. 2002, Chen and Beloborodov 2007, Proga and Zhang 2006, Perna et al. 2006, Shibata et al. 2007, Kumar et al. 2008), where photons are unable to provide cooling but neutrinos do so efficiently.

The angular momentum is quite generally a crucial parameter, in many ways determining the geometry of the flow (Lee and Ramirez-Ruiz 2006). The quasispherical approximation breaks down when the gas reaches a radius $r_{\text {circ }} \sim$ $l^{2} / G M$, where $l$ is the angular momentum per unit mass, and if injection occurs more or less isotropically at large radii, a familiar accretion disk will form. The inner regions of disks with mass fluxes $\leq \dot{M}_{\mathrm{Edd}, \nu}$ are generally able to cool by neutrinos on time scales shorter than the inflow time.

If $\dot{m}=\dot{M} / \dot{M}_{\mathrm{Edd}, \nu} \leq 1$, then the bulk of the neutrino radiation comes from a region only a few gravitational radii in size, and the physical conditions can be scaled in terms of the Eddington quantities defined above. The remaining relevant parameter, related to the angular momentum, is $v_{\text {inflow }} / v_{\text {freefall }}$, where $v_{\text {freefall }} \simeq(2 G M / R)^{1 / 2}$ is the free fall velocity. The inward drift speed $v_{\text {inflow }}$ would be of order $v_{\text {freefall }}$ for supersonic radial accretion. When angular momentum is important, this ratio depends on the mechanism for its transport through the disk, which is related to the effective shear viscosity. For a thin disk, the factor $\left(v_{\text {inflow }} / v_{\text {freefall }}\right)$ is of order $\alpha(H / R)^{2}$, where $H$ is the scale height at radius $R$ and $\alpha$ is the phenomenological viscosity parameter (Shakura and Syunyaev 1973).

The characteristic density, at a distance $r$ from the hole, with account of the effects of rotation, is

$$
\rho \sim \dot{m}\left(r / r_{\mathrm{g}}\right)^{-3 / 2}\left(v_{\text {inflow }} / v_{\text {freefall }}\right) \rho_{\text {Edd }, \nu},
$$

and the maximum magnetic field, corresponding to equipartition with the bulk kinetic energy, would be

$$
B_{\text {eq }} \sim \dot{m}^{1 / 2}\left(r / r_{\mathrm{g}}\right)^{-5 / 4}\left(v_{\text {inflow }} / v_{\text {freefall }}\right)^{1 / 2} B_{\mathrm{Edd}, \nu} .
$$

Any neutrinos emerging directly from the central core would have energies of a few $\mathrm{MeV}$. Note that, as mentioned above, $k T_{\mathrm{Edd}, \nu}$ is far below the virial temperature $k T_{\text {vir }} \simeq m_{p} c^{2}\left(r / r_{\mathrm{g}}\right)$. The flow pattern when accretion occurs would be 
then determined by the value of the parameters $L_{\nu} / L_{\mathrm{Edd}, \nu}$, which determine the importance of radiation pressure and gravity, and the ratio $t_{\mathrm{cool}} / t_{\mathrm{dynamical}}$, which fixes the temperature if a stationary flow pattern is set up.

It is widely believed that accretion onto a compact object, be it a NS or a stellar mass BH, offers the best hope of understanding the "prime mover" in all types of GRB sources although a possible attractive exception includes a rapidly spinning neutron star with a powerful magnetic field (Usov 1992, Thompson 1994, Spruit 1999, Thompson et al. 2004, Uzdensky and MacFadyen 2007, Bucciantini et al. 2008, Dessart et al. 2008). If there is an ordered field $B$, and a characteristic angular velocity $\omega$, for a spinning compact source of radius $r_{*}$, then the magnetic dipole moment is $\sim B r_{*}^{3}$. General arguments suggest (Gold 1968, Pacini 1967) that the non-thermal magnetic-dipole-like luminosity will be $L_{\mathrm{em}} \approx B^{2} r_{*}^{6} \omega^{4} / c^{3}$, and simple scaling from these familiar results of pulsar theory require fields of order $10^{15} \mathrm{G}$ to carry away the rotational or gravitational energy (which is $\sim$ $10^{53} \mathrm{erg}$ ) in a time scale of seconds (Usov 1992).

\subsection{Jet Production}

There are two ingredients necessary for the production of jets. First, there must be a source of material with sufficient free energy to escape the gravitational field of the compact object. Second, there must be a way of imparting some directionality to the escaping flow. Our eventual aim must be to understand the overall flow pattern around a central compact object, involving accretion, rotation, and directional outflow but we are still far from achieving this. Most current workers who have discussed outflow and collimation have simply invoked some central supply of energy and material. A self-consistent model incorporating outflow and inflow must explain why some fraction of the matter can acquire a disproportionate share of energy (i.e., a high enthalpy).

A spinning black hole (or neutron star) constitutes an excellent gyroscope, and the ingredients of accretion, angular momentum, entropy production, and possibly magnetic fields are probably sufficient to ensure the production of collimated outflow (McKinney 2006). However, the detailed mechanism is a matter for debate (it is not even clear what is accelerated), and several distinct lines of research are currently being pursued. One solution is to reconvert some of this energy via collisions outside the disk into electron-positron pairs or photons (Mochkovitch et al. 1993, Goodman et al. 1987, Rosswog and Ramirez-Ruiz 2002, Rosswog et al. 2003, Aloy et al. 2005, Dessart et al. 2009). If this occurs in a region of low baryon density (e.g., along the rotation axis, away from the equatorial plane of the disk) a relativistic pair-dominated wind can be produced. An obvious requirement for this mechanism to be efficient is that the neutrinos escape (free streaming, or diffusing out if the density is high enough) in a time scale shorter than that for advection into the BH. The efficiency for conversion into pairs (scaling with the square of the neutrino density) is too low if the neutrino production is too gradual, so this can become a delicate balancing act.

Jets may alternatively be produced electromagnetically. Such mechanism could, in principle, circumvent the above restriction in efficiency. The potential differ- 
ence across a disk threaded by an open magnetic field lines can exceed $10^{22} \mathrm{~V}$, and this is available for accelerating high-energy particles which will produce an electron-positron cascade and ultimately a relativistic jet that carries away the binding energy of the accreting gas. Blandford \& Znajek (1977) extended this idea to black holes and showed how the spin energy of the hole could likewise be extracted. A hydromagnetic description of this mechanism is more likely to be appropriate (Blandford and Payne 1982). The field required to produce $L_{\mathrm{em}} \geq 10^{51} \mathrm{erg} \mathrm{s}^{-1}$ is enormous, and may be provided by a helical dynamo operating in hot, convective nuclear matter with a millisecond period (Duncan and Thompson 1992). A dipole field of the order of $10^{15} \mathrm{G}$ appears weak compared to the strongest field (Price and Rosswog 2006, Thompson et al. 2005) that can in principle be generated by differential rotation $\left(\sim 10^{17}[P / 1 \mathrm{~ms}]^{-1} \mathrm{G}\right)$, or by convection $\left(\sim 10^{16} \mathrm{G}\right)$, although how this may come about in detail is not resolved. Note, however, that it only takes a residual torus (or even a cold disk) of $10^{-3} M_{\odot}$ to confine a field of $10^{15} \mathrm{G}$.

A potential death-trap for such relativistic outflows is the amount of entrained baryonic mass from the surrounding medium. For instance, a Poynting flux of $10^{53}$ erg could not accelerate an outflow to $\Gamma \geq 10^{2}$ if it had to drag more than $\sim 10^{-4} M_{\odot}$ of baryons with it. A related complication renders the production of relativistic jets even more challenging, because the high neutrino fluxes are capable of ablating baryonic material from the surface of the disk. Thus a rest mass flux $\dot{M}_{\eta}$ limits the bulk Lorentz factor of the wind to $\Gamma_{\eta}=L_{\text {wind }} /\left(\dot{M}_{\eta} c^{2}\right)$. Assuming that the external poloidal field strength is limited by the vigor of the convective motions, the spin-down luminosity scales with neutrino flux as $L_{\text {wind }} \approx L_{\mathrm{em}} \propto B^{2} \propto v_{\text {con }}^{2} \propto L_{\nu}^{2 / 3}$, where $v_{\text {con }}$ is the convective velocity. The ablation rate is $\propto L_{\nu}^{5 / 3}$ (Metzger et al. 2007, Qian and Woosley 1996), which indicates that the limiting bulk Lorentz factor $\Gamma_{\eta}$ of the wind decreases as $L_{\nu}^{-1}$. Thus the burst luminosity emitted by a magnetized neutrino cooled disk may be self-limiting. Mass loss could, however, be suppressed if the relativistic wind were somehow collimated into a jet. This suggests that centrifugally-driven mass loss will be heaviest in the outer parts of the disk, and that a detectable burst may be emitted only within a relatively small solid angle centered on the rotation axis (Levinson and Eichler 2000).

\subsection{Jet Collimation, Stability, and Confinement}

As we discussed above, one of the key issues one needs to address concerning jets is how they are formed? A second one is, how do they retain their coherence and collimation as they transverse circumburst space? The second issue is no less pressing than the first, since jet-like flows known on Earth are notoriously unstable. The situation in a jet is nonetheless different from that encountered in the laboratory because of the super-Alfvénic and supersonic streaming velocity (McKinney and Blandford 2008). MHD probably provides a better description of the macroscopic behavior of a GRB jet than in the case of laboratory plasmas because the particle Larmor radii are so much smaller than the transverse size 
of the jet. Formal stability analysis of even the simplest jet models is complex. Unstable MHD modes (such as pinch and kink instability) do not grow as rapidly as in stationary plasma, although sufficiently short-wavelength unstable modes, localized within the jet interior are not suppressed by the relative motion. Longitudinal magnetic field in the core of the jet can likewise act as a backbone, provided that the correlation length of the magnetic field reversal along the jet exceeds the wavelength of the perturbation. Instabilities may be suppressed for perturbations of large wavelength along the jet by the inertia of the ambient medium. Whatever one's view of the relative merits of fluid and electromagnetic models of relativistic jets - and perhaps the truth lies between the two extremes - it is clear that our understanding of the role of magnetic fields in jets is less advanced than that of other aspects of the problem.

An understanding of the collimation and confinement of a jet can come about only through a knowledge of the properties of the medium through which it propagates. Information about the ambient pressure gradient propagates into the jet at the internal sound speed. If the jet moves through a background with pressure scale height $\sim r$, the necessary condition for the jet interior to remain in pressure balance with its surroundings is $\theta M_{j} \leq 2$, where $M_{j}$ is the Mach number and $\theta \sim d / r$ is the opening angle. If this condition is not satisfied when a jet is moving into a region of higher pressure, then strong shocks are driven into the jet. A jet moving into a region of lower pressure becomes overpressured relative to its surroundings, and thereafter expands freely.

Freedom or confinement? This is the first question for which an extrinsic or environmental effect comes into play (which may, in turn, strongly affect what we observe), and it is thought to be particularly important for massive stellar GRB progenitors. This is because in such stars, a stellar envelope will remain to impede the advance of the jet (MacFadyen and Woosley 1999, MacFadyen et al. 2001, Mészáros and Rees 2001, Aloy et al. 2000, Ramirez-Ruiz et al. 2002a, Matzner 2003, Zhang et al. 2004, Proga et al. 2003). The beam will then evacuated a channel out to some location where it impinges on the stellar envelope. A continuous flow of relativistic fluid emanating from the nucleus supplies this region with mass, momentum, energy, and magnetic flux. Most of the energy output during that period is deposited into a cocoon or wastebasket surrounding the jet, which, after expansion, would have enough kinetic energy content to substantially alter the structure of the relativistic outflow, if not, in fact, provide much of the observed explosive power (Ramirez-Ruiz et al. 2002a).

Can relativistic jets really be formed inside stars? This is not the most propitious environment to create an ultra relativistic, baryons-starved jet. What needs to be demonstrated, is that the outflow is not "poisoned" by baryons by the time that it reaches the surface of the star. It appears that it is not necessary to collimate the jet very tightly or to achieve high bulk Lorentz factor as the flow leaves the stellar surface (Zhang et al. 2004, Morsony et al. 2007). As long as the emergent flow has a high enthalpy per baryon, it will expand and achieve its high terminal speed some distance from the star (Uzdensky and MacFadyen 2007, Bucciantini et al. 2008). A strong thermal break-out signal is expected to 
precede the canonical $\gamma$-rays observed in GRBs with massive progenitors as the shock breaks through the stellar surface and exposes the hot shocked material (MacFadyen et al. 2001, Ramirez-Ruiz et al. 2002c, Waxman and Mészáros 2003 , Waxman et al. 2007). For very extended envelopes, the jet may be unable to break through the envelope. TeV neutrino signals produced by Fermi accelerated relativistic protons within the cork may provide a means of detecting such choked-off, $\gamma$-ray dark collapses (Mészáros and Waxman 2001).

\subsection{Dissipation and Cooling Effects Within the Jet}

The unique feature of GRBs is that the bulk Lorentz factor $\Gamma$ may reach values from hundreds to thousands (Lithwick and Sari 2001). The relativistic motion of the radiating particles introduces many interesting effects in GRB emissions that must be properly taken into account (e.g. Fenimore et al. 1996b).

Three frames of reference are considered when discussing the emission from systems moving with relativistic speeds: the stationary frame, which is denoted here by asterisks, the comoving frame, denoted by primes, and the observer frame. The differential distance traveled by the expanding source during differential time $d t_{*}$ is $d r=\beta c d t_{*}$, where $\beta=\sqrt{1-\Gamma^{-2}}$. Due to time dilation, $d r=\beta \Gamma c d t^{\prime}$. The relationship between comoving and observer times is $(1+z) \Gamma d t^{\prime}(1-\beta \cos \theta)=$ $(1+z) d t^{\prime} / \delta=d t$, where $\theta$ is the angle between the emitting element and the observer, $\delta=[\Gamma(1-\beta \cos \theta)]^{-1}$ is the Doppler factor and $z$ the cosmological redshift. For an on-axis observer we therefore see that $d t \cong(1+z) d r / \Gamma^{2} c$, and, as a result, the blast wave can travel a large distance $\Gamma^{2} c \Delta t$ during a small observing time interval. A photon detected with dimensionless energy $\epsilon=h \nu / m_{e} c^{2}$ is emitted with energy $\delta \epsilon^{\prime} /(1+z)$.

Few would dispute the statement that the photons which bring us all our information about the nature of GRBs are the result of triboluminescence. For instance, velocity differences across the jet profile provide a source of free energy from particle acceleration through shock waves, hydromagnetic turbulence, and tearing mode magnetic reconnection (Rees and Mészáros 1994). If the value of $\Gamma$ at the base increases by a factor $\geq 2$ over a timescale $\Delta t$, then the later ejecta will catch up and dissipate a fraction of their energy at radius given by

$$
r_{\iota} \sim c \Delta t \Gamma^{2} \sim 3 \times 10^{14} \Delta t_{0} \Gamma_{2}^{2} \mathrm{~cm},
$$

where $\Delta t=1 \Delta t_{0} \mathrm{~s}$ and $\Gamma=10^{2} \Gamma_{2}$. Dissipation, to be most effective, must occur when the wind is optically thin: $\tau_{T} \simeq n^{\prime} \sigma_{T}(r / \Gamma) \leq 1$ (here $n^{\prime}$ is the comoving number density). Otherwise it will suffer adiabatic cooling before escaping (Goodman 1986). The photosphere (baryonic or pair-dominated) is a source of soft thermalized radiation, which may be observationally detectable in some GRB spectra and may also result in inverse Compton cooling of the nonthermal electrons accelerated in the shocks occurring outside it (Mészáros and Rees 2000, Ramirez-Ruiz and Lloyd-Ronning 2002, Spada et al. 2000, Kobayashi et al. 2002, \begin{tabular}{|l|l|l|l|}
\hline Mészáros et al. 2002, Ramirez-Ruiz 2005, Giannios and Spruit 2007, Pe'er et al. \\
\hline
\end{tabular} 2006, Ryde and Pe'er 2008, Thompson et al. 2007). 
In the presence of turbulent magnetic fields built up behind the shocks, the electrons can produce a synchrotron power-law radiation spectrum, whereas the inverse Compton scattering of these synchrotron photons extends the spectrum into the GeV range (Mészáros et al. 1994). To illustrate the basic idea, suppose that electrons, protons, and magnetic field share the available internal energy, then the electrons reach typical random Lorentz factors of $\gamma \sim m_{p} / m_{e}$; while the assumption of a Poynting flux $L_{B}$ implies a comoving magnetic field of order $B \sim L_{B}^{1 / 2} r_{\iota}^{-1} \Gamma^{-1} \sim 10^{5} L_{B, 50}^{1 / 2} r_{\iota, 13}^{-1} \Gamma_{2}^{-1} \mathrm{G}$, where $L_{B}=10^{50} L_{B, 50}$ erg s${ }^{-1}$ and $r_{\iota}=10^{13} r_{\iota, 13}$. For these values of $\gamma$ and $B$, the typical observed synchrotron frequency is $\nu_{\mathrm{sy}} \sim 0.5 L_{B, 50}^{1 / 2} r_{\iota, 13}^{-1}(1+z)^{-1} \mathrm{MeV}$, independent of the bulk Lorentz factor $\Gamma$, and in excellent agreement with the observed values of the $\nu F_{\nu}$ peak of GRB spectra (Figure 1). Yet, there are in some instances serious problems associated with this model (e.g., dissipation efficiencies). These difficulties have motivated consideration for alternative scenarios (e.g., Kumar and Narayan 2008, Kumar and McMahon 2008).

A magnetic field can ensure efficient cooling even if it is not strong enough to be dynamically significant. If, however, the field is dynamically significant in the wind (Meszaros and Rees 1997), then its internal motions could lead to dissipation even in a constant velocity wind (Thompson 1994). Instabilities in this magnetized wind may be responsible for particle acceleration (Thompson 2006), and it is possible that $\gamma$-ray production occurs mainly at large distances from the source (Lyutikov and Blandford 2003).

A further effect renders the task of simulating unsteady winds even more challenging. This stems from the likelihood that any entrained matter would be a mixture of protons and neutrons. If a streaming velocity builds up between ions and neutrons then interactions can lead to dissipation even in a steady jet where there are no shocks (Derishev et al. 1999, Beloborodov 2003).

\subsection{Jet Interaction with the External Environment}

Astrophysicists understand supernova remnants reasonably well, despite continuing uncertainty about the initiating explosion; likewise, we may hope to understand the afterglows of GRBs, despite the uncertainties about the trigger that we have already emphasized.

In the simplest version of the afterglow model, the blast wave is approximated by a uniform thin shell. A forward shock is formed when the expanding shell accelerates the external medium, and a reverse shock is formed due to deceleration of the cold shell. The forward and reverse shocked fluids are separated by a contact discontinuity and have equal kinetic energy densities.

As the blast wave expands, it sweeps up material from the surrounding medium to form an external shock (Meszaros and Rees 1993). Protons captured by the expanding blast wave from the external medium will have total energy $\Gamma m_{p} c^{2}$ in the fluid frame, where $m_{p}$ is the proton mass. The kinetic energy swept into the comoving frame by an uncollimated blast wave at the forward shock per unit time is given by (Blandford and McKee 1976) $d E^{\prime} / d t^{\prime}=4 \pi r^{2} n_{\text {ext }} m_{p} c^{3} \beta \Gamma(\Gamma-1)$, 
where factor of $\Gamma$ represents the increase of external medium density due to length contraction, the factor $(\Gamma-1)$ is proportional to the kinetic energy of the swept up particles, and the factor $\beta$ is proportional to the rate at which the particles are swept.

The external shock becomes important when the inertia of the swept-up external matter starts to produce an appreciable slowing down of the ejecta. The expanding shell will therefore begin to decelerate when $E=\Gamma M_{\mathrm{b}} c^{2}=\Gamma^{2} m_{p} c^{2}\left(4 \pi r_{\mathrm{d}}^{3} n_{\text {ext }} / 3\right)$, giving the deceleration radius (Meszaros and Rees 1993, Rees and Mészáros 1992)

$$
r_{\mathrm{d}}=\left(\frac{3 E}{4 \pi \Gamma^{2} c^{2} m_{p} n_{\mathrm{ext}}}\right)^{1 / 3} \sim 3 \times 10^{16}\left(\frac{E_{52}}{\Gamma_{2}^{2} n_{\mathrm{ext}}}\right)^{1 / 3} \mathrm{~cm},
$$

where $\Gamma \cong E / M_{\mathrm{b}} c^{2}$ is the coasting Lorentz factor, $M_{\mathrm{b}}$ is the baryonic mass, $E=10^{52} E_{52}$ erg is the apparent isotropic energy release and $n_{\text {ext }}$ is the number density of the circumburst medium. This sets a characteristic deceleration length. This deceleration allows slower ejecta to catch up, replenishing and re-energizing the reverse shock and boosting the momentum in the blast wave.

Most treatments employing blast-wave theory to explain the observed afterglow emission from GRBs assume that the radiating particles are electrons. The problem here is that $\sim m_{p} / m_{e} \sim 2000$ of the nonthermal particle energy swept into the blast-wave shock is in the form of protons or ions, unless the surroundings are composed primarily of electron-positron pairs. For a radiatively efficient system, physical processes must therefore transfer a large fraction of the sweptup energy to the electron component (Gedalin et al. 2008). In most elementary treatments it is simply assumed that a fraction $\epsilon_{e}$ of the forward-shock power is transferred to the electrons.

The strength of the magnetic field is another major uncertainty. The standard prescription is to assume that the magnetic field energy density $u_{B}$ is a fixed fraction $\epsilon_{B}$ of the downstream energy density of the shocked fluid. Hence $u_{B}=B^{2} /(8 \pi)=4 \epsilon_{B} n_{\text {ext }} m_{p} c^{2}\left(\Gamma^{2}-\Gamma\right.$ ) (although see, e.g., Rossi and Rees 2003). It is also generally supposed in simple blast-wave model calculations that some mechanism injects electrons with a power-law distribution between electron Lorentz factors $\gamma_{\min } \leq \gamma \leq \gamma_{\max }$ downstream of the shock front, where the maximum injection energy is obtained by balancing synchrotron losses and an acceleration rate given in terms of the inverse of the Larmor time scale.

A break is formed in the electron spectrum at cooling electron Lorentz factor $\gamma_{\mathrm{c}}$, which is found by balancing the synchrotron loss time scale $t_{\mathrm{sy}}^{\prime}$ with the adiabatic expansion time $t_{\text {adi }}^{\prime} \sim r /(\Gamma c)$ (Sari et al. 1998). For an adiabatic blast wave, $\Gamma \propto t^{-3 / 8}$, so that $\gamma_{\min } \propto t^{-3 / 8}$ and $\gamma_{\mathrm{c}} \propto t^{1 / 8}$. As a consequence, the accelerated electron minimum random Lorentz factor and the turbulent magnetic field also decrease as inverse power-laws in time (Meszaros and Rees 1993, Rees and Mészáros 1992). This implies that the spectrum softens in time, as the synchrotron peak corresponding to the minimum Lorentz factor and field decreases, leading to the possibility of late long wavelength emission (Sari et al. 1998).

The relativistic expansion is then gradually slowed down, and the blast wave evolves in a self-similar manner with a power-law lightcurve. This phase ends 
when so much mass shares the energy that the Lorentz factor drops to 1 Ayal and Piran 2001, Ramirez-Ruiz and MacFadyen 2008). Obviously, this happens when a mass $E / c^{2}$ has been swept up. This sets a non-relativistic mass scale:

$$
r_{\mathrm{s}}=\Gamma^{2 / 3} r_{\mathrm{d}}=\left(\frac{3 E}{4 \pi m_{p} c^{2} n_{\mathrm{ext}}}\right)^{1 / 3} \sim 10^{18}\left(\frac{E_{52}}{n_{\mathrm{ext}}}\right)^{1 / 3} \mathrm{~cm} .
$$

For comparison, the Sedov radius of a supernova that ejects a $10 M_{\odot}$ envelope could reach $\sim 5$ pc or more.

In GRB sources, with jets which we believe to be highly relativistic, the orientation of the jet axis with respect to our line of sight will strongly affect the source's appearance (Dermer 1995, Dalal et al. 2002, Granot et al. 2002, Ramirez-Ruiz et al. 2005b, Granot 2007), because radiation from jet material will be Doppler beamed in the direction of motion. Attempts to understand the luminosity function of GRBs may have to take into account the statistics of orientation, collimation,and velocity of the jet, as well as the jet's intrinsic radiation properties.

Although our proposed synthesis of GRB physical properties is highly conjectural and far from unique, we hope it will provide a framework for discussing the integrated properties of these objects. We conclude the review by discussing how future observations, experiments and theoretical studies should enhance our understanding of the physical properties underlying GRBs.

\section{PROSPECTS}

GRB studies, especially the afterglow-enabled studies of the last ten years, remain a young field. The years ahead are thus sure to bring astonishing discoveries as the capabilities and experience of observers improve, theorists make more and stronger ties to physical theory, and new and upgraded facilities open vistas. In this section, we summarize the instrumental capabilities and theoretical opportunities for near-term progress in GRB research.

\subsection{Facilities}

Table 2 summarizes recent and near-term future GRB missions, planned and proposed. Swift (Gehrels et al. 2004) is now the primary mission and has excellent prospects for continued operation, with an orbit that will be stable until at least 2020. Fermi and AGILE, also in continuing operations, are providing added burst detections with simultaneous high energy $(>100 \mathrm{MeV})$ coverage that promises to redefine the maximum energies and Lorentz factors that GRB engines are capable of producing.

The SVOM mission (Paul et al. 2008), currently under development, promises 4 to $300 \mathrm{keV}$ coverage and Swift-like slews that will bring X-ray and optical tele-

scopes to bear on burst positions. The proposed JANUS small explorer (Roming 2008) would focus on high-redshift bursts, detecting prompt emission over the 1 to $20 \mathrm{keV}$ band and slewing to observe afterglows with a near-infrared telescope 
Table 2 Recent and future GRB Missions

\begin{tabular}{|c|c|c|c|c|c|}
\hline Mission & Trigger energy range & FOV & Detector area & Other wavelengths & GRB rate $\left(\mathrm{yr}^{-1}\right)$ \\
\hline \multirow[t]{2}{*}{ BATSE } & $20 \mathrm{keV}-1.9 \mathrm{MeV}$ (LAD) & $4 \pi \mathrm{sr}$ & $2025 \mathrm{~cm}^{2}$ per LAD & & 300 \\
\hline & $10 \mathrm{keV}-100 \mathrm{MeV}$ (SD) & & $127 \mathrm{~cm}^{2}$ per $\mathrm{SD}$ & & \\
\hline HETE-2 & $6-400 \mathrm{keV}$ & $3 \mathrm{sr}$ & $120 \mathrm{~cm}^{2}$ & X-ray & \\
\hline Swift & $15-150 \mathrm{keV}$ & $1.4 \mathrm{sr}$ & $5200 \mathrm{~cm}^{2}$ & UV, Opt, X-ray & 100 ( 10\% SGRBs) \\
\hline$A G I L E$ & $30 \mathrm{MeV}-50 \mathrm{GeV}$ & $\sim 3 \mathrm{sr}$ & & Hard X-ray & \\
\hline \multirow[t]{3}{*}{ Fermi } & $20 \mathrm{MeV}-300 \mathrm{GeV}$ & $>2 \mathrm{sr}(\mathrm{LAT})$ & $>8000 \mathrm{~cm}^{2}(\mathrm{LAT})$ & & 50 \\
\hline & $8 \mathrm{keV}-1 \mathrm{MeV}$ & $9.5 \mathrm{sr}(\mathrm{GBM})$ & $126 \mathrm{~cm}^{2}$ (GBM-LED) & & \\
\hline & $150 \mathrm{keV}-30 \mathrm{MeV}$ & & $126 \mathrm{~cm}^{2}$ (GBM-HED) & & \\
\hline \multirow[t]{2}{*}{$S V O M$} & $4 \mathrm{keV}-300 \mathrm{keV}$ & $2 \mathrm{sr}(\mathrm{CXG})$ & & Optical, X-ray & 80 \\
\hline & $50 \mathrm{keV}-5 \mathrm{MeV}$ & $89^{\circ} \times 89^{\circ}(\mathrm{GRM})$ & & & \\
\hline JANUS & $1-20 \mathrm{keV}$ & $4 \mathrm{sr}$ & & Near-IR & 25 (high $z$ ) \\
\hline EXIST & $5-600 \mathrm{keV}$ & $\sim 3.6 \mathrm{sr}(\mathrm{HET})$ & $5.96 \mathrm{~m}^{2}(\mathrm{HET})$ & $\begin{array}{l}\text { Optical, near-IR, } \\
\text { X-ray }\end{array}$ & 300 \\
\hline
\end{tabular}

Note: LAD, Large Area Detector; SD, Spectroscopy Detector; LAT, Large Area Telescope; LED, Low Energy Detector; HED, High Energy Detector; CSG, X-ray/Gamma-ray Camera; GRM, Gamma-Ray Monitor; GBM, GLAST Burst Monitor; SGRB, short GRB.

(50 $\mathrm{cm}$ aperture, 0.7 to $1.7 \mu \mathrm{m}$ coverage). The proposed large mission EXIST has also added fast-response slews and focusing X-ray and near-infrared telescopes to its original complement of hard X-ray imaging detectors (Grindlay 2008).

Current and near-future ground-based facilities include a fast-growing array of robotic telescopes primed to respond to burst alerts, ongoing improvements to the instrumentation and capabilities of large-aperture telescopes, a new generation of air-Cerenkov $\mathrm{TeV}$ facilities, and with the EVLA initiative, the most significant upgrade to a high-sensitivity radio facility in decades.

Rapid follow-up of GRB discoveries is occurring both in space and on the ground; the Swift UVOT observes $\sim 80$ GRBs per year within 2 minutes, detecting about half of them. On the ground, fast new telescopes such as ROTSE-III (Akerlof et al. 2003), RAPTOR (Woźniak et al. 2006), Pi-of-the-sky (Burd et al. 2005), and REM (Zerbi et al. 2001) are able to point at GRBs within $10-20 \mathrm{~s}$, and larger facilities like the seven-band GROND (Greiner et al.2008) respond on 10-minute timescales. Ready availability of sensitive CCDs and $\mathrm{HgCdTe}$ detectors, combined with rapid-slew mounts and autonomous software systems, should enable further expansion of rapid-response telescopes with more larger $(D \gtrsim 2 \mathrm{~m})$ facilities anticipated in the near future.

GRB-related programs continue to compete successfully for time on premier optical facilities. Large-aperture $(D \gtrsim 6 \mathrm{~m})$ telescopes provide the spectroscopic observations necessary for GRB redshift measurements. At the VLT, a rapid response mode has gone further to provide time-sequence observations revealing variable absorption from the host galaxy, and the impending commissioning of the X-Shooter spectrograph (Kaper et al. 2009) will enable full UV to NIR characterization of afterglow spectra with a single integration. In addition to the TOO opportunities provided by GRB alerts, multiple host galaxy survey programs are underway.

At radio wavelengths, the workhorse facilities have been the VLA (e.g., Chandra et al. 2008) and WSRT (e.g., van der Horst et al. 2007), providing data primarily in the $1-10 \mathrm{GHz}$ at sensitivities in the $0.2-1.0 \mathrm{mJy}$ range; the 
Expanded VLA (EVLA) upgrade will improve the sensitivity of that facility to $\sim 10 \mu \mathrm{Jy}$. Looking ahead, by 2012, the ALMA array will be on line operating in the higher frequency range $90-950 \mathrm{GHz}$ with $>100$ times the sensitivity of the VLA. The peak in the synchrotron spectrum for a wide range of GRB parameters lies in the ALMA range, and should make it a powerful future tool for radio observations.

Observations of GRBs at $\mathrm{TeV}$ energies can be performed by both narrowfield air Cerenkov facilities and wide-field water Cerenkov detectors; the latter approach, at Milagro, yielded the tentative detection of prompt $\mathrm{TeV}$ emission for GRB 970417A (Atkins et al. 2003). There has been no detection to-date of prompt or afterglow emission with the air Cerenkov facilities, but significant effort in pursuit of burst alerts is underway at MAGIC, HESS, and VERITAS. The MAGIC dish, in particular, has a rapid-response mode that has provided observations of multiple bursts within a minute of trigger (Albert et al. 2006). Future facilties will seek to lower detection thresholds to $E \sim 100 \mathrm{GeV}$, which would provide a significantly-expanded horizon within which GRB sources will be visible, rather than attenuated by photon-photon interactions.

In space, the upcoming HST SM4 promises to revive that facility, not only providing new and resuscitated instruments, but also the gyros necessary for flexible and fast-response scheduling. In the future, the capabilities of JWST will provide an excellent resource for high signal-to-noise observations of highly obscured and high-redshift GRBs.

Within the X-ray band, the Swift XRT has redefined all expectations for the characterization of X-ray afterglows, and at the same time, proved a highly effective facility for refining the multi-arcmin localizations provided by other GRB missions. Chandra, XMM, and Suzaku continue to be active in GRB observations, and future missions including the Indian ASTROSAT and Japan/US Astro-H promise added capabilities in the near future. Dramatic improvements will await the next-generation GRB facilities (Table 2) or the arrival of the International $X$-ray Observatory.

\subsection{Multimessenger Aspects}

Given the rapid ongoing expansion of the capabilities of non-electromagnetic detector facilities, and the extreme luminosity and time-specificity of GRB sources, it will not be long before we either have the first coincident, multi-messenger detection of a GRB, or realize limits on the non-electromagnetic emissions of GRBs that challenge our current understanding.

The same shocks which are thought to accelerate electrons responsible for the non-thermal $\gamma$-rays in GRBs should also accelerate protons, leading ultimately to copious emission of high-energy neutrinos (Waxman 2004b, Waxman 2004c, Waxman 2006, Dermer and Holmes 2005). The maximum proton energies achievable in GRB shocks are $E_{p} \sim 10^{20} \mathrm{eV}$, comparable to the highest energies measured with large cosmic ray ground arrays (Hayashida et al. 1999). For this, the acceleration time must be shorter than both the radiation or adiabatic loss time and the escape time from the acceleration region (Waxman 1995). The accelerated 
protons can interact with the fireball photons, leading to charged pions, muons and neutrinos. For internal shocks producing observed $1 \mathrm{MeV}$ photons this implies $\geq 10^{16} \mathrm{eV}$ protons, and neutrinos with $\sim 5 \%$ of that energy, $\epsilon_{\nu} \geq 10^{14} \mathrm{eV}$ (Waxman and Bahcall 1997). Another copious source of target photons in the $\mathrm{UV}$ is the afterglow reverse shock, for which the resonance condition requires higher energy protons leading to neutrinos of $10^{17}-10^{19} \mathrm{eV}$ (Waxman and Bahcall 1999). Whereas photon-pion interactions lead to higher energy neutrinos and provide a direct probe of the shock proton acceleration as well as of the photon density, inelastic proton-neutron collisions may occur even in the absence of shocks, leading to charged pions and neutrinos (Derishev et al. 1999) with lower energies than those from photon-pion interactions. The typical neutrino energies are in the $\sim 1-10 \mathrm{GeV}$ range, which could be detectable in coincidence with observed GRBs. This is the province of projects like AMANDA, IceCube and ANTARES. Neutrino astronomy has the advantage that we can see the universe up to $\sim$ EeV energies. By contrast, the universe becomes opaque to $\gamma$-rays above $\sim \mathrm{TeV}$ energies through absorption by the infrared background.

The last and most challenging frontier is that of gravitational radiation, which is largely unknown territory. A time-integrated luminosity of the order of a solar rest mass $\left(\sim 10^{54} \mathrm{erg}\right)$ is predicted from progenitor models involving merging compact objects, while that from collapsar models is less certain, and expected to be lower by at least one order of magnitude.

Ground-based facilities, like LIGO, TAMA and VIRGO, are currently seeking the first detection of these stellar-scale, high-frequency $(\nu \gtrsim 50 \mathrm{~Hz})$ sources. The observation of associated gravitational waves would be facilitated if the mergers involve observed short GRB sources; and conversely, it may be possible to strengthen the case for (or against) NS-NS or NS-BH progenitors of short bursts if gravitational waves were detected (or not) in coincidence with some events. The technical challenge of achieving the sensitivities necessary to measure waves from assured sources should not be understated; neither, however, should the potential rewards.

The Enhanced LIGO interferometers will be on line in 2009 with ability to detect NS binary mergers to $20 \mathrm{Mpc}$. The Advanced LIGO interferometers on line in 2014 will extend the distance to $200 \mathrm{Mpc}$. Short GRBs, if produced by mergers as is thought to be the case, predict a cosmological rate density of $>10$ $\mathrm{Gpc}^{-1} \mathrm{yr}^{-1}$ with likely rate of $\sim 300 \mathrm{Gpc}^{-1} \mathrm{yr}^{-1}$ (O'Shaughnessy et al. 2005, O'Shaughnessy et al. 2007, O'Shaughnessy et al. 2008b, O'Shaughnessy et al. 2008a, Nakar 2007b). This translates into an Advanced LIGO detection rate $\sim 10 \mathrm{yr}^{-1}$. The density estimates include mergers with $\gamma$-ray jets not aimed in our direction, so not all LIGO detection would be in coincidence with GRBs. LIGO is already providing useful upper limits, as with GRB 070201 described in Section III.

\subsection{Cosmology}

One of the frontiers of modern cosmology lies at high redshift, $z \gtrsim 6$, when the first non-linearities developed into gravitationally-bound systems, whose internal 
evolution gives rise to stars, galaxies, and quasars; and when the light emitted from these first collapsed structures diffuse outwards to reionize the Universe. As the (temporarily) brightest source of photons in the cosmos (see Figure 15), the demise of these first generations of massive stars in GRB explosions defines the challenge of elucidating the end of the cosmic "dark ages" (Lamb and Reichart 2000, Bromm and Loeb 2002).

Apart from revealing a site of high-redshift star-formation, each such highredshift burst has the potential to help constrain local element abundances in its host galaxy, information that will be impossible to gather by other means until the advent of $D \gtrsim 20 \mathrm{~m}$ telescopes. Even more exciting, each burst has the potential to reveal the extent of intergalactic reionization, at its redshift and along that line of sight (Miralda-Escudé 1998, McQuinn et al. 2008, Mesinger and Furlanetto 2008).

At the highest redshifts, $z \gtrsim 10$, there is growing theoretical evidence indicating that the first luminous objects to form were very massive stars $M>100 M_{\odot}$. Depending on whether these stars retain their high masses until death, and whether a fast-rotating core is prerequisite to the GRB phenomenon, these Pop III stars might provide the progenitors for the most luminous, highest-redshift GRBs (Heger et al. 2003).

The first GRBs and supernovae may also be important for another reason: they may generate the first cosmic magnetic fields. Mass loss (e.g. via winds) would disperse magnetic flux along with the heavy elements. The ubiquity of heavy elements in the Lyman alpha forest indicates that there has been widespread diffusion from the sites of these early supernovae, and the magnetic flux could have diffused in the same way. This flux, stretched and sheared by bulk motions, could be the "seed" for the later amplification processes that generate the largerscale fields pervading disc galaxies.

\subsection{Theoretical Prospects}

What can we expect in the wave of matching theoretical progress? This is more difficult to disucss because theory often develops on a shorter timescale than observations and experiments, and so we cannot forsee most future developments. Although some of the features now observed in GRB sources (especially afterglows) were anticipated by theoretical discussions, the recent burst of observational discovery has left theory lagging behind. There are, however, some topics on which we do believe that there will be steady work of direct relevance to interpreting observations.

One of the most important is the development and use of hydrodynamical codes for numerical simulation of GRB sources with detailed physics input. Existing two and three dimensional codes have already uncovered some gas-dynamical properties of relativistic flows unanticipated by analytical models (McKinney and Blandford 2008) but there are some key questions that they cannot yet address. In particular, higher resolution is needed because even a tiny mass fraction of baryons loading down the outflow severely limits the maximum attainable Lorentz factor. What is more, jets are undoutedly susceptible to hydrostatic and hydro- 


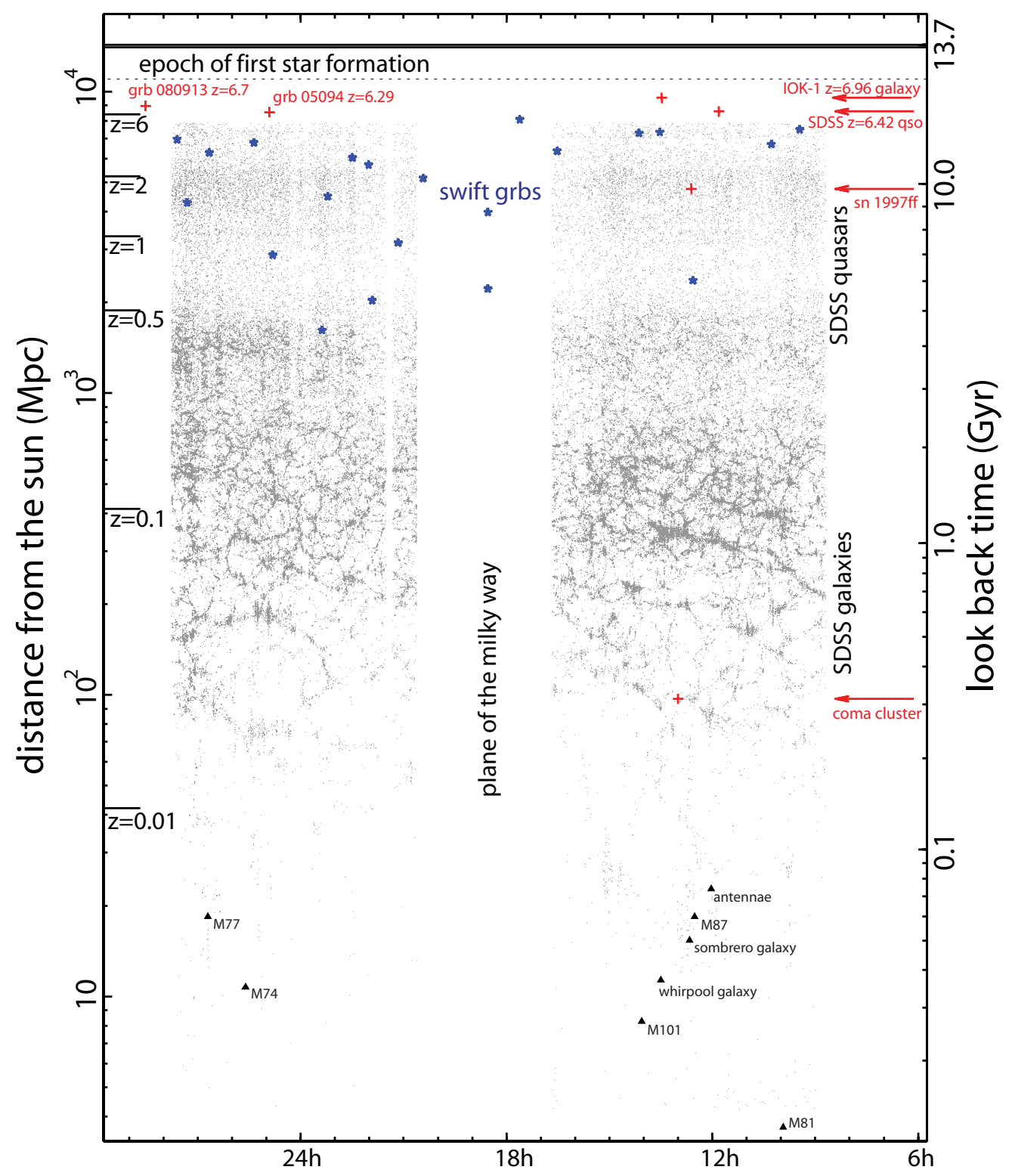

Figure 15 A $360^{\circ}$ vista showing the entire sky, with visible structures stretching back in distance, time and redshift. The most distant light we observe comes from the radiation left over from the Big Bang: the CMB. As we descend the chart, we find the most distant objects known, followed by a web of Sloan Digital Sky Survey (SDSS) quasars and galaxies. Closer to home, we start to see a collection of familiar "near" galaxies (triangles). Also marked are all Swift GRBs with known distances (blue stars); SN 1997ff, the most distant type Ia supernova at $z=1.7$; and the archetypal large galaxy cluster, the Coma cluster. The redshift distances of most distant GRBs are comparable to the most distant galaxies and quasars (adapted from Ramirez-Ruiz 2006a.) 
magnetic turbulence. We must wait for useful and affordable three dimensional simulations before we can understand the nonlinear development of instabilities. Well-resolved three dimensional simulations are becoming increasingly common and they rarely fail to surprise us. The symmetry-breaking involved in transitioning from two to three dimensions is crucial and can lead to qualitatively new phenomena. A particularly important aspect of this would be to link in a self-consistent manner the flow within the accretion disk to that in the outflowing gas, allowing for feedback between the two components. A self-consistent model incorporating inflow and outflow must also explain how some fraction of the material can acquire more than its share of energy (i.e., a high enthalpy or $p / \rho)$.

Particle acceleration and cooling is another problem that seems ripe for a more sophisticated treatment. Few would dispute the statement that the photons which bring us all our information about the nature of GRBs are the result of particle acceleration in relativistic shocks (Medvedev and Spitkovsky 2008, Spitkovsky 2008, Ramirez-Ruiz et al. 2007, Katz et al. 2007) or turbulence (Goodman and MacFadyen 2007, Zhang et al. 2009, Couch et al. 2008). Since charged particles radiate only when accelerated, one must attempt to deduce from the spectrum how the particles are being accelerated, why they are being accelerated, and to identify the macroscopic source driving the microphysical acceleration process.

Collisionless shocks are among the main agents for accelerating ions as well as electrons to high energies whenever sufficient time is available (e.g., Blandford and Eichler 1987, Achterberg et al. 2001). Particles reflected from the shock and from scattering centres behind it in the turbulent compressed region have a good chance of experiencing multiple scattering and acceleration by first-order Fermi acceleration when coming back across the shock into the turbulent upstream region. Second-order or stochastic Fermi acceleration in the broadband turbulence downstream of collisionless shocks will also contribute to acceleration. In addition, ions may be trapped at perpendicular shocks. The trapping is a consequence of the shock and the Lorentz force exerted on the particle by the magnetic and electric fields in the upstream region. With each reflection at the shock the particles gyrate parallel to the motional electric field, picking up energy and surfing along the shock surface. All these mechanisms are still under investigation, but there is evidence that shocks play a most important role in the acceleration of cosmic rays and other particles to very high energies.

Another topic on which further work seems practicable concerns the kinematics of ultrarelativistic jets (Granot 2007). While it seems probable that we are using the correct ingredients of special relativity and a collimated outflow, it is equally true that no detailed model yet commands majority of support. We can still expect some surprises from studies related to the appearence of relativistic shocks in unsteady jets.

The most interesting problem remains, however, in the nature of the central engine and the means of extracting power in a useful collimated form. In all observed cases of relativistic jets, the central object is compact, either a neutron star or black hole, and is accreting matter and angular momentum. In addition, in 
most systems there is direct or indirect evidence that magnetic fields are present - detected in the synchrotron radiation in galactic and extragalactic radio sources or inferred in collapsing supernova cores from the association of remnants with radio pulsars. This combination of magnetic field and rotation may be very relevant to the production of relativistic jets.

\section{CONCLUSIONS}

Thanks primarily to the burst discoveries and observations of the Swift satellite, the last five years have been tremendously productive ones for GRB research. The identification of short burst afterglows has confirmed long-held suspicions that GRBs have at least two fundamentally different types of progenitors; subsequent studies of short burst afterglows and host galaxies have furnished valuable information on the nature of their progenitors and provided hopeful indications for next-generation gravity-wave observatories. The discovery of the first two bursts at $z>6$, before sources reionized most of the hydrogen in the universe, has proven the value of GRBs as probes of the earliest cosmic epochs and and extended GRB observations beyond the redshifts of the most distant known quasars (see Figure 16). A flood of prompt burst alerts has fed the queues of more than a dozen dedicated robotic telescopes, and prompted fast-response multi-epoch high-resolution spectroscopy from the largest telescopes. Overall, bright GRBs continue to attract the attention of astronomers of all types, with premier facilities across the electromagnetic spectrum poised to respond to the next spectacular event. In the near future, we hope that gravity-wave and high-energy neutrino astronomers will be rewarded for their decades of persistent effort.

The complexity - not to mention sheer volume - of data in the Swift era have inevitably raised challenges to the prior interpretation of bursts and their afterglows. However, careful consideration of the biases inherent to the Swift mission observing strategies, along with the large number of events now available for analysis, are gradually enabling the construction of a single coherent picture, via a multidisciplinary approach that addresses data from across the electromagnetic spectrum. It is one of the challenges of contemporary research to infer the underlying physical structure of GRBs in the belief that this is simple, despite the complex character of the observations.

GRBs provide us with an exciting opportunity to study new regimes of physics. As we have described, our rationalization of the principal physical considerations combines some generally accepted features with some more speculative and controversial ingredients. When confronted with observations, it seems to accommodate their gross features but fails to provide us with a fully predictive theory. What is more valuable, though considerably harder to achieve, is to refine models like the ones advocated here to the point of making quantitative predictions, and to assemble, assess and interpret observations so as to constrain and refute these theories. What we can hope of our present understanding is that it will assist us in this endeavour.

There are good prospects for a continued high rate of discovery going forward. 


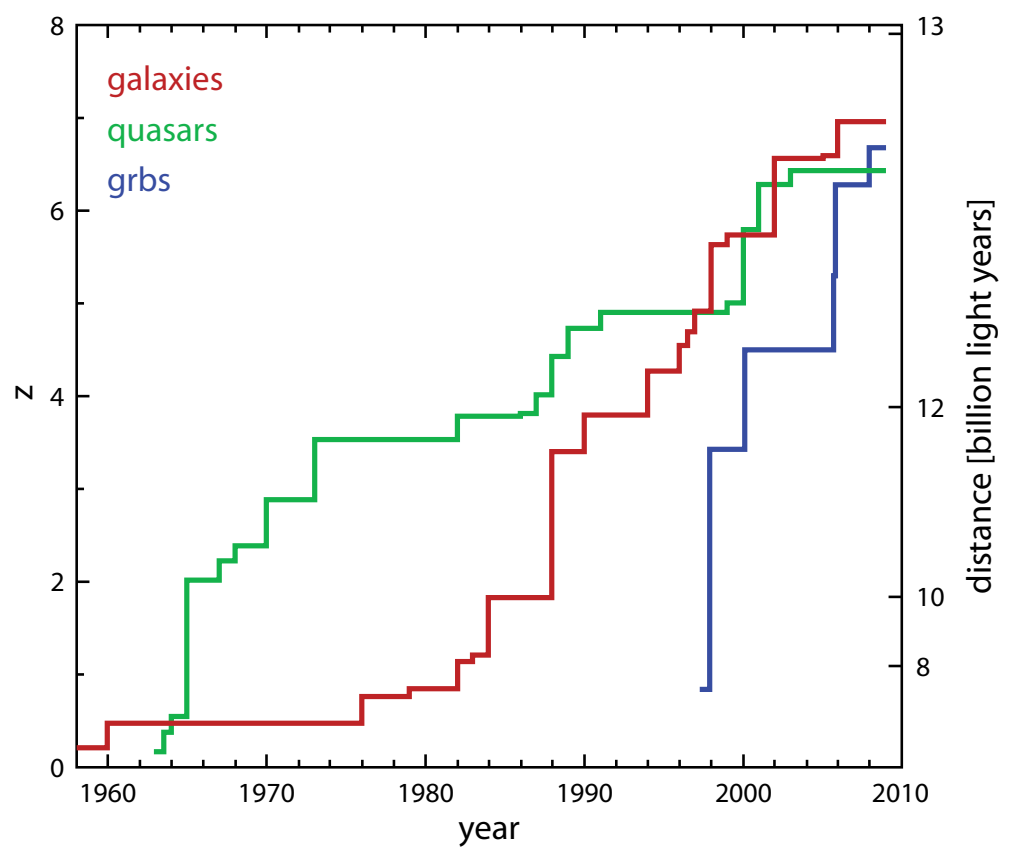

Figure 16 High-z record holders. The history of most distant objects detected in categories of galaxies, quasars, and GRBs (R. McMahon, N. Tanvir, priv. comm.).

Swift is likely to remain operational for many years and Fermi and AGILE, now on orbit, are providing new insights at higher energies. Instruments currently in development or planning have the potential to enable further qualitative advances. GRBs are among the most extraordinary of astronomical phenomena and will, with our present and future capabilities, continue providing a unique window into the extreme reaches of the Universe.

\section{Acknowledgements}

We are indebted to many colleagues for their contributions to this review. We are very grateful to John Cannizzo for his insightful comments and tireless help with editing the manuscript. Several colleagues provided figures and data that we much appreciate. They are Edo Berger, Andy Fruchter, Alexander Kahn, Yuki Kaneko, David Morris, Kim Page, Mario Juric, Jacob Palmatier, Bryan Penprase, J. X. Prochaska, Judy Racusin, Taka Sakamoto, Richard McMahon, and Nial Tanvir. Our views on the topics discussed here have been clarified through discussions with Josh Bloom, Jonathan Granot, William Lee, Peter Meszaros, Udi Nakar, Tsvi Piran, X. Prochaska, Chryssa Kouveliotou, Pawan Kumar, Edo Berger, Alicia Soderberg, Brad Cenko, Dale Frail, Jane Charlton, David Burrows, Doug Cowen, Richard O'Shaughnessy, Kris Belczynski, Kris Stanek, Matthew McQuinn, Hsiao-Wen Chen, Andrew MacFadyen, Sam Oates, Martin J. Rees, Stephan Rosswog, Eli Waxman, Stan Woosley, Chris Fryer, Guillermo GarciaSegura, Norbert Langer, Sung-Chul Yoon, Dan Kasen, and Annalisa Celotti. We thank J. D. Myers and Rion Parsons for assistance with graphics. Finally, we 
thank the 200 members of the Swift team for the wonderful new findings reported here.

This work is partially supported by NSF: PHY-0503584 (ER-R), DOE SciDAC: DE-FC02-01ER41176 (ER-R).

\section{References}

Abbott, B., Abbott, R., Adhikari, R., Ajith, P., Allen, B., et al., 2008, ApJ 681, 1419

Achterberg, A., Gallant, Y. A., Kirk, J. G., and Guthmann, A. W., 2001, MNRAS 328,393

Akerlof, C., Balsano, R., Barthelemy, S., Bloch, J., Butterworth, P., et al., 1999, Nature 398, 400

Akerlof, C. W., Kehoe, R. L., McKay, T. A., Rykoff, E. S., Smith, D. A., et al., 2003, PASP 115, 132

Albert, J., Aliu, E., Anderhub, H., Antoranz, P., Armada, A., et al., 2006, ApJ 641, L9

Aloy, M. A., Janka, H.-T., and Müller, E., 2005, A\&A 436, 273

Aloy, M. A., Müller, E., Ibáñez, J. M., Martí, J. M., and MacFadyen, A., 2000, ApJ 531, L119

Amati, L., 2006, MNRAS 372, 233

Amati, L., Frontera, F., Tavani, M., in't Zand, J. J. M., Antonelli, A., et al., 2002, A\&A 390, 81

Antonelli, L. A., Piro, L., Vietri, M., Costa, E., Soffitta, P., et al., 2000, ApJ 545, L39

Atkins, R., Benbow, W., Berley, D., Chen, M. L., Coyne, D. G., et al., 2003, ApJ 583,824

Atwood, W. B. et al., 2009, ApJ in prep,

Ayal, S. and Piran, T., 2001, ApJ 555, 23

Baring, M. G. and Harding, A. K., 1997, ApJ 491, 663

Barraud, C., Olive, J.-F., Lestrade, J. P., Atteia, J.-L., Hurley, K., et al., 2003, A\&A 400, 1021

Barthelmy, S. D., Barbier, L. M., Cummings, J. R., Fenimore, E. E., Gehrels, N., et al., 2005a, Space Science Reviews 120, 143

Barthelmy, S. D., Cannizzo, J. K., Gehrels, N., Cusumano, G., Mangano, V., et al., 2005b, ApJ 635, L133

Barthelmy, S. D., Chincarini, G., Burrows, D. N., Gehrels, N., Covino, S., et al., 2005c, Nature 438, 994

Belczynski, K., Bulik, T., and Rudak, B., 2002, ApJ 571, 394

Belczynski, K., Perna, R., Bulik, T., Kalogera, V., Ivanova, N., et al., 2006, ApJ 648,1110 
Beloborodov, A. M., 2003, ApJ 585, L19

Berger, E., 2009, ApJ 690, 231

Berger, E., Fox, D. B., Kulkarni, S. R., Frail, D. A., and Djorgovski, S. G., 2007a, ApJ 660, 504

Berger, E., Fox, D. B., Price, P. A., Nakar, E., Gal-Yam, A., et al., 2007b, ApJ 664,1000

Berger, E., Penprase, B. E., Fox, D. B., Kulkarni, S. R., Hill, G., et al., 2005a, ArXiv Astrophysics e-prints

Berger, E., Price, P. A., Cenko, S. B., Gal-Yam, A., Soderberg, A. M., et al., 2005b, Nature 438, 988

Bhat, P. N., Fishman, G. J., Meegan, C. A., Wilson, R. B., Brock, M. N., et al., 1992, Nature 359, 217

Blake, C. H., Bloom, J. S., Starr, D. L., Falco, E. E., Skrutskie, M., et al., 2005, Nature 435, 181

Blandford, R. and Eichler, D., 1987, Phys. Rep. 154, 1

Blandford, R. D. and McKee, C. F., 1976, Physics of Fluids 19, 1130

Blandford, R. D. and Payne, D. G., 1982, MNRAS 199, 883

Blandford, R. D. and Znajek, R. L., 1977, MNRAS 179, 433

Bloom, J. S., Frail, D. A., and Kulkarni, S. R., 2003, ApJ 594, 674

Bloom, J. S., Frail, D. A., and Sari, R., 2001, AJ 121, 2879

Bloom, J. S., Kulkarni, S. R., and Djorgovski, S. G., 2002, AJ 123, 1111

Bloom, J. S., Perley, D. A., Chen, H.-W., Butler, N., Prochaska, J. X., et al., 2007, ApJ 654, 878

Bloom, J. S., Perley, D. A., Li, W., Butler, N. R., Miller, A. A., et al., 2009, ApJ 691,723

Bloom, J. S. and Prochaska, J. X., 2006, in S. S. Holt, N. Gehrels, and J. A. Nousek (eds.), Gamma-Ray Bursts in the Swift Era, Vol. 836 of American Institute of Physics Conference Series, p. 473

Bloom, J. S., Prochaska, J. X., Pooley, D., Blake, C. H., Foley, R. J., et al., 2006, ApJ 638, 354

Blustin, A. J., Band, D., Barthelmy, S., Boyd, P., Capalbi, M., et al., 2006, ApJ 637, 901

Boër, M., Atteia, J. L., Damerdji, Y., Gendre, B., Klotz, A., and Stratta, G., 2006, ApJ 638, L71

Bromm, V. and Loeb, A., 2002, ApJ 575, 111

Bucciantini, N., Quataert, E., Arons, J., Metzger, B. D., and Thompson, T. A., 2008, MNRAS 383, L25

Burd, A., Cwiok, M., Czyrkowski, H., Dabrowski, R., Dominik, W., et al., 2005, New Astronomy 10, 409 
Burrows, D. N., Hill, J. E., Nousek, J. A., Kennea, J. A., Wells, A., et al., 2005a, Space Science Reviews 120, 165

Burrows, D. N., Romano, P., Falcone, A., Kobayashi, S., Zhang, B., et al., 2005b, Science 309, 1833

Castro-Tirado, A. J., de Ugarte Postigo, A., Gorosabel, J., Fathkullin, T., Sokolov, V., Bremer, M., Márquez, I., Marín, A. J., Guziy, S., Jelínek, M., Kubánek, P., Hudec, R., Vitek, S., Mateo Sanguino, T. J., Eigenbrod, A., Pérez-Ramírez, M. D., Sota, A., Masegosa, J., Prada, F., and Moles, M., 2005, A\&A 439, L15

Cenko, S. B., Berger, E., Nakar, E., Kasliwal, M. M., Cucchiara, A., et al., 2008, ArXiv e-prints

Cenko, S. B., Kasliwal, M., Harrison, F. A., Pal'shin, V., Frail, D. A., et al., 2006, ApJ 652, 490

Chandra, P., Cenko, S. B., Frail, D. A., Chevalier, R. A., Macquart, J.-P., et al., 2008, ApJ 683, 924

Chary, R., Berger, E., and Cowie, L., 2007, ApJ 671, 272

Chen, H.-W., Perley, D. A., Pollack, L. K., Prochaska, J. X., Bloom, J. S., et al., 2008, ArXiv e-prints

Chen, H.-W., Prochaska, J. X., Bloom, J. S., and Thompson, I. B., 2005, ApJ 634, L25

Chen, H.-W., Prochaska, J. X., Ramirez-Ruiz, E., Bloom, J. S., DessaugesZavadsky, M., et al., 2007, ApJ 663, 420

Chen, W.-X. and Beloborodov, A. M., 2007, ApJ 657, 383

Chincarini, G., Moretti, A., Romano, P., Falcone, A. D., Morris, D., et al., 2007, ApJ 671, 1903

Christensen, L., Hjorth, J., and Gorosabel, J., 2004, A\&A 425, 913

Cline, T. L. and Desai, U. D., 1974, NASA STI/Recon Technical Report N 75, 11888

Connaughton, V., 2002, ApJ 567, 1028

Conselice, C. J., Vreeswijk, P. M., Fruchter, A. S., Levan, A., Kouveliotou, C., et al., 2005, ApJ 633, 29

Couch, S. M., Milosavljević, M., and Nakar, E., 2008, ApJ 688, 462

Courty, S., Björnsson, G., and Gudmundsson, E. H., 2004, MNRAS 354, 581

Courty, S., Björnsson, G., and Gudmundsson, E. H., 2007, MNRAS 376, 1375

Covino, S., Malesani, D., Israel, G. L., D’Avanzo, P., Antonelli, L. A., et al., 2006, A\&A 447, L5

Cusumano, G., Mangano, V., Chincarini, G., Panaitescu, A., Burrows, D. N., et al., 2006, Nature 440, 164

Dai, X., Garnavich, P. M., Prieto, J. L., Stanek, K. Z., Kochanek, C. S., et al., 2008, ApJ 682, L77 
Dai, X., Halpern, J. P., Morgan, N. D., Armstrong, E., Mirabal, N., et al., 2007, ApJ 658, 509

Dalal, N., Griest, K., and Pruet, J., 2002, ApJ 564, 209

D’Avanzo, P., Malesani, D., Covino, S., Piranomonte, S., Grazian, A., et al., 2009, ArXiv e-prints

D'Elia, V., Fiore, F., Perna, R., Krongold, Y., Covino, S., et al., 2008, ArXiv e-prints

Della Valle, M., Chincarini, G., Panagia, N., Tagliaferri, G., Malesani, D., et al., 2006a, Nature 444, 1050

Della Valle, M., Chincarini, G., Panagia, N., Tagliaferri, G., Malesani, D., Testa, V., Fugazza, D., Campana, S., Covino, S., Mangano, V., Antonelli, L. A., D'Avanzo, P., Hurley, K., Mirabel, I. F., Pellizza, L. J., Piranomonte, S., and Stella, L., 2006b, Nature 444, 1050

Derishev, E. V., Kocharovsky, V. V., and Kocharovsky, V. V., 1999, ApJ 521, 640

Dermer, C. D., 1995, ApJ 446, L63+

Dermer, C. D. and Holmes, J. M., 2005, ApJ 628, L21

Dessart, L., Burrows, A., Livne, E., and Ott, C. D., 2008, ApJ 673, L43

Dessart, L., Ott, C. D., Burrows, A., Rosswog, S., and Livne, E., 2009, ApJ 690, 1681

Di Matteo, T., Perna, R., and Narayan, R., 2002, ApJ 579, 706

Duncan, R. C. and Thompson, C., 1992, ApJ 392, L9

Evans, P. A., Beardmore, A. P., Page, K. L., Osborne, J. P., O'Brien, P. T., et al., 2008, ArXiv e-prints

Evans, P. A., Beardmore, A. P., Page, K. L., Tyler, L. G., Osborne, J. P., et al., 2007, A\&A 469, 379

Falcone, A. D., Morris, D., Racusin, J., Chincarini, G., Moretti, A., et al., 2007, ApJ 671, 1921

Fenimore, E. E., Epstein, R. I., and Ho, C., 1993, A\&AS 97, 59

Fenimore, E. E., Klebesadel, R. W., and Laros, J. G., 1996a, ApJ 460, 964

Fenimore, E. E., Madras, C. D., and Nayakshin, S., 1996b, ApJ 473, 998

Fenimore, E. E., Palmer, D., Galassi, M., Tavenner, T., Barthelmy, S., et al., 2003, in G. R. Ricker and R. K. Vanderspek (eds.), Gamma-Ray Burst and Afterglow Astronomy 2001: A Workshop Celebrating the First Year of the HETE Mission, Vol. 662 of American Institute of Physics Conference Series, p. 491

Fenimore, E. E. and Ramirez-Ruiz, E., 2000, ArXiv Astrophysics e-prints

Firmani, C., Ghisellini, G., Avila-Reese, V., and Ghirlanda, G., 2006, MNRAS 370, 185 
Foley, R. J., Bloom, J. S., Prochaska, J. X., Illingworth, G. D., Holden, B. P., et al., 2005, GRB Coordinates Network 4409, 1

Fox, A. J., Ledoux, C., Vreeswijk, P. M., Smette, A., and Jaunsen, A. O., 2008, A\&A 491, 189

Fox, D. B., Frail, D. A., Price, P. A., Kulkarni, S. R., Berger, E., et al., 2005, Nature 437, 845

Fox, D. B. and Roming, P. W. A., 2007, Royal Society of London Philosophical Transactions Series A 365, 1293

Frail, D. A., Cameron, P. B., Kasliwal, M., Nakar, E., Price, P. A., et al., 2006, ApJ 646, L99

Frail, D. A., Kulkarni, S. R., Nicastro, L., Feroci, M., and Taylor, G. B., 1997, Nature 389, 261

Frail, D. A., Kulkarni, S. R., Sari, R., Djorgovski, S. G., Bloom, J. S., et al., 2001, ApJ 562, L55

Freedman, D. L. and Waxman, E., 2001, ApJ 547, 922

Fruchter, A. S., Levan, A. J., Strolger, L., Vreeswijk, P. M., Thorsett, S. E., et al., 2006, Nature 441, 463

Fryer, C. L., Woosley, S. E., and Hartmann, D. H., 1999, ApJ 526, 152

Fynbo, J. P. U., Jakobsson, P., Möller, P., Hjorth, J., Thomsen, B., et al., 2003, A\&A 406, L63

Fynbo, J. P. U., Prochaska, J. X., Sommer-Larsen, J., Dessauges-Zavadsky, M., and Møller, P., 2008, ApJ 683, 321

Fynbo, J. P. U., Starling, R. L. C., Ledoux, C., Wiersema, K., Thöne, C. C., et al., 2006a, A\&A 451, L47

Fynbo, J. P. U., Watson, D., Thöne, C. C., Sollerman, J., Bloom, J. S., et al., 2006b, Nature 444, 1047

Gaensler, B. M., Kouveliotou, C., Gelfand, J. D., Taylor, G. B., Eichler, D., et al., 2005, Nature 434, 1104

Gal-Yam, A., Fox, D. B., Price, P. A., Ofek, E. O., Davis, M. R., et al., 2006, Nature 444, 1053

Gal-Yam, A., Nakar, E., Ofek, E. O., Cenko, S. B., Kulkarni, S. R., et al., 2008, ApJ 686, 408

Galama, T. J., Vreeswijk, P. M., van Paradijs, J., Kouveliotou, C., Augusteijn, T., et al., 1998, Nature 395, 670

Gedalin, M., Balikhin, M. A., and Eichler, D., 2008, Phys. Rev. E 77(2), 026403

Gehrels, N., Barthelmy, S. D., Burrows, D. N., Cannizzo, J. K., Chincarini, G., et al., 2008, ApJ 689, 1161

Gehrels, N., Chincarini, G., Giommi, P., Mason, K. O., Nousek, J. A., et al., 2004, ApJ 611, 1005 
Gehrels, N., Norris, J. P., Barthelmy, S. D., Granot, J., Kaneko, Y., et al., 2006, Nature 444, 1044

Gehrels, N., Sarazin, C. L., O'Brien, P. T., Zhang, B., Barbier, L., et al., 2005, Nature 437, 851

Ghirlanda, G., Ghisellini, G., and Lazzati, D., 2004, ApJ 616, 331

Giannios, D. and Spruit, H. C., 2007, A\&A 469, 1

Gold, T., 1968, Nature 218, 731

Goodman, J., 1986, ApJ 308, L47

Goodman, J., Dar, A., and Nussinov, S., 1987, ApJ 314, L7

Goodman, J. and MacFadyen, A. I., 2007, ArXiv e-prints

Gou, L.-J., Fox, D. B., and Mészáros, P., 2007, ApJ 668, 1083

Granot, J., 2007, in Revista Mexicana de Astronomia y Astrofisica, vol. 27, Vol. 27 of Revista Mexicana de Astronomia y Astrofisica Conference Series, pp $140-165$

Granot, J., 2008, ArXiv e-prints

Granot, J., Cohen-Tanugi, J., and do Couto e Silva, E., 2008, ApJ 677, 92

Granot, J. and Kumar, P., 2006, MNRAS 366, L13

Granot, J., Panaitescu, A., Kumar, P., and Woosley, S. E., 2002, ApJ 570, L61

Granot, J. and Ramirez-Ruiz, E., 2004, ApJ 609, L9

Granot, J., Ramirez-Ruiz, E., and Loeb, A., 2005a, ApJ 618, 413

Granot, J., Ramirez-Ruiz, E., and Perna, R., 2005b, ApJ 630, 1003

Greiner, J., Bornemann, W., Clemens, C., Deuter, M., Hasinger, G., et al., 2008, PASP 120, 405

Grindlay, J., 2008, in 37th COSPAR Scientific Assembly, Vol. 37 of COSPAR, Plenary Meeting, p. 1084

Grupe, D., Gronwall, C., Wang, X.-Y., Roming, P. W. A., Cummings, J., et al., 2007, ApJ 662, 443

Guetta, D., Granot, J., and Begelman, M. C., 2005, ApJ 622, 482

Guetta, D. and Piran, T., 2006, A\&A 453, 823

Guetta, D. and Piran, T., 2007, Journal of Cosmology and Astro-Particle Physics 7,3

Guilbert, P. W., Fabian, A. C., and Rees, M. J., 1983, MNRAS 205, 593

Haislip, J. B., Nysewander, M. C., Reichart, D. E., Levan, A., Tanvir, N., et al., 2006, Nature 440, 181

Hayashida, N., Nagano, M., Nishikawa, D., Ohoka, H., Sakaki, N., et al., 1999, Astroparticle Physics 10, 303

Heger, A., Fryer, C. L., Woosley, S. E., Langer, N., and Hartmann, D. H., 2003, ApJ 591, 288 
Heise, J., in't Zand, J., Kippen, R. M., and Woods, P. M., 2001, in E. Costa, F. Frontera, and J. Hjorth (eds.), Gamma-ray Bursts in the Afterglow Era, p. 16

Hjorth, J., Sollerman, J., Gorosabel, J., Granot, J., Klose, S., Kouveliotou, C., Melinder, J., Ramirez-Ruiz, E., Starling, R., Thomsen, B., Andersen, M. I., Fynbo, J. P. U., Jensen, B. L., Vreeswijk, P. M., Castro Cerón, J. M., Jakobsson, P., Levan, A., Pedersen, K., Rhoads, J. E., Tanvir, N. R., Watson, D., and Wijers, R. A. M. J., 2005a, ApJ 630, L117

Hjorth, J., Sollerman, J., Møller, P., Fynbo, J. P. U., Woosley, S. E., et al., 2003, Nature 423,847

Hjorth, J., Watson, D., Fynbo, J. P. U., Price, P. A., Jensen, B. L., et al., 2005b, Nature 437, 859

Horack, J. M., Koshut, T. M., Mallozzi, R. S., Storey, S. D., and Emslie, A. G., 1994, ApJ 429, 319

Houck, J. C. and Chevalier, R. A., 1991, ApJ 376, 234

Hurkett, C. P., Vaughan, S., Osborne, J. P., O’Brien, P. T., Page, K. L., et al., 2008, ApJ 679, 587

Hurley, K., Boggs, S. E., Smith, D. M., Duncan, R. C., Lin, R., et al., 2005, Nature 434, 1098

Hurley, K., Cline, T., Mazets, E., Barthelmy, S., Butterworth, P., et al., 1999, Nature 397, 41

Izzard, R. G., Ramirez-Ruiz, E., and Tout, C. A., 2004, MNRAS 348, 1215

Jakobsson, P., Levan, A., Fynbo, J. P. U., Priddey, R., Hjorth, J., et al., 2006, A\&A 447, 897

Kaneko, Y., González, M. M., Preece, R. D., Dingus, B. L., and Briggs, M. S., 2008, ApJ 677, 1168

Kaneko, Y., Ramirez-Ruiz, E., Granot, J., Kouveliotou, C., Woosley, S. E., et al., 2007, ApJ 654, 385

Kann, D. A., Klose, S., Zhang, B., Malesani, D., Nakar, E., et al., 2007, ArXiv e-prints

Kaper, L., D’Odorico, S., Hammer, F., Pallavicini, R., Kjaergaard Rasmussen, P., et al., 2009, in A. Moorwood (ed.), Science with the VLT in the ELT Era, p. 319

Katz, B., Keshet, U., and Waxman, E., 2007, ApJ 655, 375

Katz, J. I., 1994, ApJ 422, 248

Katz, J. I. and Canel, L. M., 1996, ApJ 471, 915

Kawabata, K. S., Deng, J., Wang, L., Mazzali, P., Nomoto, K., et al., 2003, ApJ 593, L19

Kawai, N., Kosugi, G., Aoki, K., Yamada, T., Totani, T., et al., 2006, Nature 440,184 
Kehoe, R., Akerlof, C., Balsano, R., Barthelmy, S., Bloch, J., et al., 2001, ApJ 554, L159

Kippen, R. M., Woods, P. M., Heise, J., in’t Zand, J. J. M., Briggs, M. S., et al., 2003, in G. R. Ricker and R. K. Vanderspek (eds.), AIP Conf. Proc. 662: Gamma-Ray Burst and Afterglow Astronomy 2001: A Workshop Celebrating the First Year of the HETE Mission, p. 244

Kistler, M. D., Yüksel, H., Beacom, J. F., and Stanek, K. Z., 2008, ApJ 673, L119

Klebesadel, R. W., Strong, I. B., and Olson, R. A., 1973, ApJ 182, L85

Klotz, A., Gendre, B., Stratta, G., Atteia, J. L., Boër, M., et al., 2006, A\&A 451, L39

Kluzniak, W. and Lee, W. H., 1998, ApJ 494, L53+

Kobayashi, S., Ryde, F., and MacFadyen, A., 2002, ApJ 577, 302

Kobulnicky, H. A. and Kewley, L. J., 2004, ApJ 617, 240

Kocevski, D. and Butler, N., 2008, ApJ 680, 531

Kouveliotou, C., Meegan, C. A., Fishman, G. J., Bhat, N. P., Briggs, M. S., et al., 1993, ApJ 413, L101

Kuiper, L., Hermsen, W., Cusumano, G., Diehl, R., Schönfelder, V., et al., 2001, A\&A 378,918

Kumar, P. and McMahon, E., 2008, MNRAS 384, 33

Kumar, P. and Narayan, R., 2008, ArXiv e-prints

Kumar, P., Narayan, R., and Johnson, J. L., 2008, MNRAS 388, 1729

Kumar, P. and Panaitescu, A., 2000, ApJ 541, L51

Kumar, P. and Panaitescu, A., 2008, MNRAS 391, L19

Lamb, D. Q. and Reichart, D. E., 2000, ApJ 536, 1

Lamb, D. Q., Ricker, G. R., Atteia, J.-L., Barraud, C., Boer, M., et al., 2004, New Astronomy Review 48, 423

Lazzati, D., Ramirez-Ruiz, E., and Ghisellini, G., 2001, A\&A 379, L39

Le, T. and Dermer, C. D., 2007, ApJ 661, 394

Le Floc'h, E., Charmandaris, V., Forrest, W. J., Mirabel, I. F., Armus, L., et al., 2006, ApJ 642, 636

Le Floc'h, E., Duc, P.-A., Mirabel, I. F., Sanders, D. B., Bosch, G., et al., 2003, A\&A 400, 499

Lee, W. H. and Ramirez-Ruiz, E., 2006, ApJ 641, 961

Lee, W. H. and Ramirez-Ruiz, E., 2007, New Journal of Physics 9, 17

Lee, W. H., Ramirez-Ruiz, E., and Granot, J., 2005a, ApJ 630, L165

Lee, W. H., Ramirez-Ruiz, E., and Page, D., 2004, ApJ 608, L5

Lee, W. H., Ramirez-Ruiz, E., and Page, D., 2005b, ApJ 632, 421 
Levinson, A. and Eichler, D., 2000, Physical Review Letters 85, 236

Lithwick, Y. and Sari, R., 2001, ApJ 555, 540

Lloyd-Ronning, N. M. and Ramirez-Ruiz, E., 2002, ApJ 576, 101

Lopez-Camara, D., Lee, W. H., and Ramirez-Ruiz, E., 2008, ArXiv e-prints

Lyutikov, M. and Blandford, R., 2003, ArXiv Astrophysics e-prints

MacFadyen, A. I. and Woosley, S. E., 1999, ApJ 524, 262

MacFadyen, A. I., Woosley, S. E., and Heger, A., 2001, ApJ 550, 410

Matheson, T., Garnavich, P. M., Stanek, K. Z., Bersier, D., Holland, S. T., et al., 2003, ApJ 599, 394

Matsuoka, M., Kawai, N., Yoshida, A., Tamagawa, T., Torii, K., et al., 2004, Baltic Astronomy 13, 201

Matzner, C. D., 2003, MNRAS 345, 575

Mazets, E. P., Aptekar, R. L., Cline, T. L., Frederiks, D. D., Goldsten, J. O., et al., 2008, ApJ 680, 545

Mazets, E. P., Golenetskii, S. V., Ilyinskii, V. N., Panov, V. N., Aptekar, R. L., et al., 1981, Ap\&SS 80, 85

McConnell, M. L., Zdziarski, A. A., Bennett, K., Bloemen, H., Collmar, W., et al., 2002, ApJ 572, 984

McKinney, J. C., 2006, MNRAS 368, 1561

McKinney, J. C. and Blandford, R. D., 2008, ArXiv e-prints

McQuinn, M., Lidz, A., Zaldarriaga, M., Hernquist, L., and Dutta, S., 2008, MNRAS 388, 1101

Medvedev, M. V. and Spitkovsky, A., 2008, ArXiv e-prints

Mesinger, A. and Furlanetto, S. R., 2008, MNRAS 385, 1348

Mészáros, P., 2002, ARA\&A 40, 137

Mészáros, P., Ramirez-Ruiz, E., Rees, M. J., and Zhang, B., 2002, ApJ 578, 812

Meszaros, P. and Rees, M. J., 1993, ApJ 405, 278

Mészáros, P. and Rees, M. J., 1997, ApJ 476, 232

Meszaros, P. and Rees, M. J., 1997, ApJ 482, L29+

Mészáros, P. and Rees, M. J., 1999, MNRAS 306, L39

Mészáros, P. and Rees, M. J., 2000, ApJ 530, 292

Mészáros, P. and Rees, M. J., 2001, ApJ 556, L37

Mészáros, P., Rees, M. J., and Papathanassiou, H., 1994, ApJ 432, 181

Mészáros, P. and Waxman, E., 2001, Physical Review Letters 87(17), 171102

Metzger, B. D., Piro, A. L., and Quataert, E., 2008, MNRAS 390, 781

Metzger, B. D., Thompson, T. A., and Quataert, E., 2007, ApJ 659, 561

Metzger, M. R., Djorgovski, S. G., Kulkarni, S. R., Steidel, C. C., Adelberger, K. L., et al., 1997, Nature 387, 878 
Mirabal, N., Halpern, J. P., Chornock, R., Filippenko, A. V., Terndrup, D. M., et al., 2003, ApJ 595, 935

Miralda-Escudé, J., 1998, ApJ 501, 15

Mochkovitch, R., Hernanz, M., Isern, J., and Martin, X., 1993, Nature 361, 236

Modjaz, M., Kewley, L., Kirshner, R. P., Stanek, K. Z., Challis, P., et al., 2008, AJ 135, 1136

Molinari, E., Vergani, S. D., Malesani, D., Covino, S., D’Avanzo, P., et al., 2007, A\&A 469, L13

Möller, P., Fynbo, J. P. U., Hjorth, J., Thomsen, B., Egholm, M. P., et al., 2002, A\&A 396, L21

Morsony, B. J., Lazzati, D., and Begelman, M. C., 2007, ApJ 665, 569

Nakar, E., 2007a, Phys. Rep. 442, 166

Nakar, E., 2007b, Advances in Space Research 40, 1224

Nakar, E., Gal-Yam, A., and Fox, D. B., 2006a, ApJ 650, 281

Nakar, E., Gal-Yam, A., Piran, T., and Fox, D. B., 2006b, ApJ 640, 849

Narayan, R., Paczyński, B., and Piran, T., 1992, ApJ 395, L83

Narayan, R., Piran, T., and Kumar, P., 2001, ApJ 557, 949

Norris, J. P. and Bonnell, J. T., 2006, ApJ 643, 266

Norris, J. P., Cline, T. L., Desai, U. D., and Teegarden, B. J., 1984, Nature 308, 434

Norris, J. P. and Gehrels, N., 2008, in M. Galassi, D. Palmer, and E. Fenimore (eds.), American Institute of Physics Conference Series, Vol. 1000 of American Institute of Physics Conference Series, p. 280

Norris, J. P., Nemiroff, R. J., Bonnell, J. T., Scargle, J. D., Kouveliotou, C., et al., 1996, ApJ 459, 393

Nousek, J. A., Kouveliotou, C., Grupe, D., Page, K. L., Granot, J., et al., 2006, ApJ 642, 389

Nysewander, M., Fruchter, A. S., and Pe'er, A., 2008, ArXiv e-prints

Oates, S. R., Page, M. J., Schady, P., de Pasquale, M., Koch, T. S., et al., 2009, MNRAS pp 376-+

O'Brien, P. T., Willingale, R., Osborne, J., Goad, M. R., Page, K. L., et al., 2006, ApJ 647, 1213

Ofek, E. O., Cenko, S. B., Gal-Yam, A., Fox, D. B., Nakar, E., et al., 2007, ApJ 662,1129

Oren, Y., Nakar, E., and Piran, T., 2004, MNRAS 353, L35

O'Shaughnessy, R., Belczynski, K., and Kalogera, V., 2008a, ApJ 675, 566

O'Shaughnessy, R., Kalogera, V., and Belczynski, K., 2007, ApJ 667, 1048

O’Shaughnessy, R., Kim, C., Fragos, T., Kalogera, V., and Belczynski, K., 2005, ApJ 633, 1076 
O’Shaughnessy, R., Kim, C., Kalogera, V., and Belczynski, K., 2008b, ApJ 672, 479

Pacini, F., 1967, Nature 216, 567

Paczyński, B., 1986, ApJ 308, L43

Paczyński, B., 1990, ApJ 363, 218

Paczyński, B., 1998, ApJ 494, L45

Paczyński, B. and Xu, G., 1994, ApJ 427, 708

Palmer, D. M., Barthelmy, S., Gehrels, N., Kippen, R. M., Cayton, T., et al., 2005, Nature 434, 1107

Panaitescu, A., Mészáros, P., Burrows, D., Nousek, J., Gehrels, N., et al., 2006, MNRAS 369, 2059

Paul, J., Wei, J., Zhang, S., and Basa, S., 2008, in 37th COSPAR Scientific Assembly, Vol. 37 of COSPAR, Plenary Meeting, p. 2368

Pe'er, A., Mészáros, P., and Rees, M. J., 2006, ApJ 642, 995

Penprase, B. E., Berger, E., Fox, D. B., Kulkarni, S. R., Kadish, S., et al., 2006, ApJ 646, 358

Perna, R., Armitage, P. J., and Zhang, B., 2006, ApJ 636, L29

Pian, E., Mazzali, P. A., Masetti, N., Ferrero, P., Klose, S., et al., 2006, Nature 442, 1011

Pihlström, Y. M., Taylor, G. B., Granot, J., and Doeleman, S., 2007, ApJ 664, 411

Piran, T., Kumar, P., Panaitescu, A., and Piro, L., 2001, ApJ 560, L167

Piran, T., Sari, R., and Zou, Y.-C., 2009, MNRAS 393, 1107

Piran, T. and Shemi, A., 1993, ApJ 403, L67

Piro, L., Costa, E., Feroci, M., Frontera, F., Amati, L., et al., 1999, ApJ 514, L73

Piro, L., Garmire, G., Garcia, M., Stratta, G., Costa, E., et al., 2000, Science 290, 955

Podsiadlowski, P., Mazzali, P. A., Nomoto, K., Lazzati, D., and Cappellaro, E., 2004, ApJ 607, L17

Porciani, C. and Madau, P., 2001, ApJ 548, 522

Porciani, C., Viel, M., and Lilly, S. J., 2007, ApJ 659, 218

Preece, R. D., Briggs, M. S., Mallozzi, R. S., Pendleton, G. N., Paciesas, W. S., et al., 2000, ApJS 126, 19

Price, D. J. and Rosswog, S., 2006, Science 312, 719

Price, P. A., Songaila, A., Cowie, L. L., Bell Burnell, J., Berger, E., et al., 2007, ApJ 663, L57

Prochaska, J. X., Chen, H.-W., and Bloom, J. S., 2006, ApJ 648, 95 
Prochaska, J. X., Chen, H.-W., Bloom, J. S., Dessauges-Zavadsky, M., O'Meara, J. M., et al., 2007, ApJS 168, 231

Prochaska, J. X., Dessauges-Zavadsky, M., Ramirez-Ruiz, E., and Chen, H.-W., 2008, ApJ 685, 344

Prochaska, J. X., Sheffer, Y., Perley, D. A., Bloom, J. S., Lopez, L. A., et al., 2009, ApJ 691, L27

Prochter, G. E., Prochaska, J. X., Chen, H.-W., Bloom, J. S., DessaugesZavadsky, M., et al., 2006, ApJ 648, L93

Proga, D., MacFadyen, A. I., Armitage, P. J., and Begelman, M. C., 2003, ApJ 599, L5

Proga, D. and Zhang, B., 2006, MNRAS 370, L61

Qian, Y.-Z. and Woosley, S. E., 1996, ApJ 471, 331

Quimby, R. M., Rykoff, E. S., Yost, S. A., Aharonian, F., Akerlof, C. W., et al., 2006a, ApJ 640, 402

Quimby, R. M., Rykoff, E. S., Yost, S. A., Aharonian, F., Akerlof, C. W., et al., 2006b, ApJ 640, 402

Racusin, J. L., Karpov, S. V., Sokolowski, M., Granot, J., Wu, X. F., et al., 2008a, Nature 455, 183

Racusin, J. L., Liang, E. W., Burrows, D. N., Falcone, A., Sakamoto, T., et al., 2008b, arXiv.org 0812.4780

Ramirez-Ruiz, E., 2005, MNRAS 363, L61

Ramirez-Ruiz, E., 2006a, Nature 440, 154

Ramirez-Ruiz, E., 2006b, Nuovo Cimento B Serie 121, 1261

Ramirez-Ruiz, E., Celotti, A., and Rees, M. J., 2002a, MNRAS 337, 1349

Ramirez-Ruiz, E. and Fenimore, E. E., 2000, ApJ 539, 712

Ramirez-Ruiz, E., García-Segura, G., Salmonson, J. D., and Pérez-Rendón, B., 2005a, ApJ 631, 435

Ramirez-Ruiz, E., Granot, J., Kouveliotou, C., Woosley, S. E., Patel, S. K., and Mazzali, P. A., 2005b, ApJ 625, L91

Ramirez-Ruiz, E., Lazzati, D., and Blain, A. W., 2002b, ApJ 565, L9

Ramirez-Ruiz, E. and Lloyd-Ronning, N. M., 2002, New Astronomy 7, 197

Ramirez-Ruiz, E. and MacFadyen, A. I., 2008, ArXiv e-prints

Ramirez-Ruiz, E., MacFadyen, A. I., and Lazzati, D., 2002c, MNRAS 331, 197

Ramirez-Ruiz, E., Nishikawa, K.-I., and Hededal, C. B., 2007, ApJ 671, 1877

Rees, M. J. and Mészáros, P., 1992, MNRAS 258, 41P

Rees, M. J. and Mészáros, P., 1994, ApJ 430, L93

Reeves, J. N., Watson, D., Osborne, J. P., Pounds, K. A., O'Brien, P. T., et al., 2002, Nature 416, 512 
Reichart, D. E., Lamb, D. Q., Fenimore, E. E., Ramirez-Ruiz, E., Cline, T. L., and Hurley, K., 2001, ApJ 552, 57

Rhoads, J. E., 1999, ApJ 525, 737

Romano, P., Campana, S., Mignani, R. P., Moretti, A., Mottini, M., et al., 2008, VizieR Online Data Catalog 348, 81221

Roming, P., 2008, in 37th COSPAR Scientific Assembly, Vol. 37 of COSPAR, Plenary Meeting, p. 2645

Roming, P. W. A., Kennedy, T. E., Mason, K. O., Nousek, J. A., Ahr, L., et al., 2005, Space Science Reviews 120, 95

Roming, P. W. A., Koch, T. S., Oates, S. R., Porterfield, B. L., Vanden Berk, D. E., et al., 2009, ApJ 690, 163

Rossi, E. and Rees, M. J., 2003, MNRAS 339, 881

Rosswog, S. and Liebendörfer, M., 2003, MNRAS 342, 673

Rosswog, S. and Ramirez-Ruiz, E., 2002, MNRAS 336, L7

Rosswog, S., Ramirez-Ruiz, E., and Davies, M. B., 2003, MNRAS 345, 1077

Rosswog, S., Speith, R., and Wynn, G. A., 2004, MNRAS 351, 1121

Ruffert, M., Janka, H.-T., Takahashi, K., and Schaefer, G., 1997, A\&A 319, 122

Ryde, F. and Pe'er, A., 2008, ArXiv e-prints

Rykoff, E. S., Aharonian, F., Akerlof, C. W., Alatalo, K., Ashley, M. C. B., et al., 2005a, ApJ 631, 1032

Rykoff, E. S., Mangano, V., Yost, S. A., Sari, R., Aharonian, F., et al., 2006a, ApJ 638, L5

Rykoff, E. S., Mangano, V., Yost, S. A., Sari, R., Aharonian, F., et al., 2006b, ApJ 638, L5

Rykoff, E. S., Smith, D. A., Price, P. A., Akerlof, C. W., Ashley, M. C. B., et al., 2004a, ApJ 601, 1013

Rykoff, E. S., Smith, D. A., Price, P. A., Akerlof, C. W., Ashley, M. C. B., et al., 2004b, ApJ 601, 1013

Rykoff, E. S., Yost, S. A., Krimm, H. A., Aharonian, F., Akerlof, C. W., et al., 2005b, ApJ 631, L121

Sakamoto, T., Barbier, L., Barthelmy, S. D., Cummings, J. R., Fenimore, E. E., et al., 2006, ApJ 636, L73

Sakamoto, T., Lamb, D. Q., Kawai, N., Yoshida, A., Graziani, C., et al., 2005, ApJ 629, 311

Salvaterra, R., Cerutti, A., Chincarini, G., Colpi, M., Guidorzi, C., et al., 2008, MNRAS 388, L6

Sari, R., Narayan, R., and Piran, T., 1996, ApJ 473, 204

Sari, R. and Piran, T., 1999, ApJ 517, L109

Sari, R., Piran, T., and Narayan, R., 1998, ApJ 497, L17+ 
Savaglio, S., Glazebrook, K., and LeBorgne, D., 2009, ApJ 691, 182

Schaefer, B. E., 2006, ApJ 642, L25

Schaefer, B. E., Gerardy, C. L., Höflich, P., Panaitescu, A., Quimby, R., et al., 2003, ApJ 588, 387

Setiawan, S., Ruffert, M., and Janka, H.-T., 2004, MNRAS 352, 753

Setiawan, S., Ruffert, M., and Janka, H.-T., 2006, A\&A 458, 553

Shakura, N. I. and Syunyaev, R. A., 1973, A\&A 24, 337

Shaviv, N. J. and Dar, A., 1995, MNRAS 277, 287

Shibata, M., Sekiguchi, Y., and Takahashi, R., 2007, Progress of Theoretical Physics 118, 257

Shin, M.-S. and Berger, E., 2007, ApJ 660, 1146

Soderberg, A. M., Berger, E., Kasliwal, M., Frail, D. A., Price, P. A., et al., 2006a, ApJ 650, 261

Soderberg, A. M., Berger, E., Page, K. L., Schady, P., Parrent, J., et al., 2008, Nature 453, 469

Soderberg, A. M., Kulkarni, S. R., Berger, E., Fox, D. B., Price, P. A., et al., 2004, ApJ 606, 994

Soderberg, A. M., Kulkarni, S. R., Nakar, E., Berger, E., Cameron, P. B., et al., 2006b, Nature 442, 1014

Spada, M., Panaitescu, A., and Mészáros, P., 2000, ApJ 537, 824

Spitkovsky, A., 2008, ApJ 673, L39

Spruit, H. C., 1999, A\&A 341, L1

Stanek, K. Z., Dai, X., Prieto, J. L., An, D., Garnavich, P. M., et al., 2007, ApJ 654, L21

Stanek, K. Z., Gnedin, O. Y., Beacom, J. F., Gould, A. P., Johnson, J. A., et al., 2006, Acta Astronomica 56, 333

Stanek, K. Z., Matheson, T., Garnavich, P. M., Martini, P., Berlind, P., et al., 2003, ApJ 591, L17

Tagliaferri, G., Antonelli, L. A., Chincarini, G., Fernández-Soto, A., Malesani, D., et al., 2005a, A\&A 443, L1

Tagliaferri, G., Goad, M., Chincarini, G., Moretti, A., Campana, S., et al., 2005b, Nature 436, 985

Tanvir, N. R., Chapman, R., Levan, A. J., and Priddey, R. S., 2005, Nature 438, 991

Taylor, G. B., Frail, D. A., Berger, E., and Kulkarni, S. R., 2004, ApJ 609, L1

Taylor, G. B., Momjian, E., Pihlström, Y., Ghosh, T., and Salter, C., 2005, ApJ 622,986

Thompson, C., 1994, MNRAS 270, 480

Thompson, C., 2006, ApJ 651, 333 
Thompson, C., Mészáros, P., and Rees, M. J., 2007, ApJ 666, 1012

Thompson, T. A., Chang, P., and Quataert, E., 2004, ApJ 611, 380

Thompson, T. A., Quataert, E., and Burrows, A., 2005, ApJ 620, 861

Thöne, C. C., Fynbo, J. P. U., Östlin, G., Milvang-Jensen, B., Wiersema, K., et al., 2008, ApJ 676, 1151

Tremonti, C. A., Heckman, T. M., Kauffmann, G., Brinchmann, J., Charlot, S., White, S. D. M., Seibert, M., Peng, E. W., Schlegel, D. J., Uomoto, A., Fukugita, M., and Brinkmann, J., 2004, ApJ 613, 898

Tumlinson, J., Prochaska, J. X., Chen, H.-W., Dessauges-Zavadsky, M., and Bloom, J. S., 2007, ApJ 668, 667

Usov, V. V., 1992, Nature 357, 472

Uzdensky, D. A. and MacFadyen, A. I., 2007, ApJ 669, 546

van der Horst, A. J., Kamble, A., Wijers, R. A. M. J., Resmi, L., Bhattacharya, D., et al., 2007, Royal Society of London Philosophical Transactions Series A 365,1241

van Paradijs, J., Groot, P. J., Galama, T., Kouveliotou, C., Strom, R. G., et al., 1997, Nature 386, 686

van Paradijs, J., Kouveliotou, C., and Wijers, R. A. M. J., 2000, ARA\&A 38, 379

Vestrand, W. T., Woźniak, P. R., Wren, J. A., Fenimore, E. E., Sakamoto, T., et al., 2005, Nature 435, 178

Vestrand, W. T., Wren, J. A., Woźniak, P. R., Aptekar, R., Golentskii, S., et al., 2006, Nature 442, 172

Villasenor, J. S., Lamb, D. Q., Ricker, G. R., Atteia, J.-L., Kawai, N., et al., 2005, Nature 437, 855

Vreeswijk, P. M., Ellison, S. L., Ledoux, C., Wijers, R. A. M. J., Fynbo, J. P. U., et al., 2004, A\&A 419, 927

Vreeswijk, P. M., Ledoux, C., Smette, A., Ellison, S. L., Jaunsen, A. O., et al., 2007, A\&A 468, 83

Wainwright, C., Berger, E., and Penprase, B. E., 2007, ApJ 657, 367

Waxman, E., 1995, Physical Review Letters 75, 386

Waxman, E., 2004a, ApJ 605, L97

Waxman, E., 2004b, New Journal of Physics 6, 140

Waxman, E., 2004c, ApJ 606, 988

Waxman, E., 2006, Nuclear Physics B Proceedings Supplements 151, 46

Waxman, E. and Bahcall, J., 1997, Physical Review Letters 78, 2292

Waxman, E. and Bahcall, J., 1999, Phys. Rev. D 59(2), 023002

Waxman, E., Kulkarni, S. R., and Frail, D. A., 1998, ApJ 497, 288

Waxman, E. and Mészáros, P., 2003, ApJ 584, 390 
Waxman, E., Mészáros, P., and Campana, S., 2007, ApJ 667, 351

Willingale, R., O'Brien, P. T., Osborne, J. P., Godet, O., Page, K. L., et al., 2007, ApJ 662, 1093

Woods, E. and Loeb, A., 1995, ApJ 453, 583

Woosley, S. E., 1993, ApJ 405, 273

Woosley, S. E. and Bloom, J. S., 2006, ARA\&A 44, 507

Woźniak, P. R., Vestrand, W. T., Panaitescu, A. D., Wren, J. A., Davis, H. R., et al., 2008, ArXiv e-prints

Woźniak, P. R., Vestrand, W. T., Wren, J. A., White, R. R., Evans, S. M., et al., 2006, ApJ 642, L99

Yoshida, A., Namiki, M., Otani, C., Kawai, N., Murakami, T., et al., 1999, A\&AS 138,433

Yost, S. A., Alatalo, K., Rykoff, E. S., Aharonian, F., Akerlof, C. W., et al., 2006a, ApJ 636, 959

Yost, S. A., Alatalo, K., Rykoff, E. S., Aharonian, F., Akerlof, C. W., et al., 2006b, ApJ 636, 959

Yüksel, H., Kistler, M. D., Beacom, J. F., and Hopkins, A. M., 2008, ApJ 683, L5

Zerbi, R. M., Chincarini, G., Ghisellini, G., Rondonó, M., Tosti, G., et al., 2001, Astronomische Nachrichten 322, 275

Zhang, B., 2007, Chinese Journal of Astronomy and Astrophysics 7, 1

Zhang, B., Fan, Y. Z., Dyks, J., Kobayashi, S., Mészáros, P., et al., 2006, ApJ 642,354

Zhang, B., Kobayashi, S., and Mészáros, P., 2003, ApJ 595, 950

Zhang, W., MacFadyen, A., and Wang, P., 2009, ApJ 692, L40

Zhang, W., Woosley, S. E., and Heger, A., 2004, ApJ 608, 365

Zheng, Z. and Ramirez-Ruiz, E., 2007, ApJ 665, 1220

Zou, Y.-C., Piran, T., and Sari, R., 2009, ApJ 692, L92 